\title{
O Teorema da Aplicação de Riemann: uma prova livre de integração
}

\author{
Jéssica Laís Calado de Barros \\ DissertaÇÃo APRESENTADA \\ $\mathrm{AO}$ \\ Instituto De Matemática e Estatística \\ DA \\ Universidade De SÃo Paulo \\ PARA \\ OBTENÇÃO DO TÍTULO \\ $\mathrm{DE}$ \\ Mestre em CiÊnCIAS
}

Programa: Matemática

Orientador: Prof. Dr. Oswaldo Rio Branco de Oliveira

Durante o desenvolvimento deste trabalho o autor recebeu auxílio financeiro do $\mathrm{CNPq}$

São Paulo, fevereiro de 2016 


\section{O Teorema da Aplicação de Riemann: uma prova livre de integração}

Esta versão da dissertação contém as correções e alterações sugeridas pela Comissão Julgadora durante a defesa da versão original do trabalho, realizada em 08/04/2016. Uma cópia da versão original está disponível no

Instituto de Matemática e Estatística da Universidade de São Paulo.

Comissão Julgadora:

- Prof. Dr. Oswaldo Rio Branco de Oliveira (orientador) - IME-USP

- Prof. Dr. Paulo Agozzini Martin - IME-USP

- Prof. Dr. Ernandes Rocha de Oliveira - FEIS - UNESP 


\section{Agradecimentos}

Agradeço primeiramente a Deus, sem Ele nada disso seria possível.

Agradeço também aos meus pais Oziel e Cristina pelo apoio durante toda a minha vida acadêmica e por serem pais tão maravilhosos, sempre cuidando de mim com muito amor, e às minhas queridas irmãs Jully e Emilly pelos momentos que me fizeram sorrir e pelas diversas alegrias proporcionadas.

Agradeço ao meu orientador por ser muito atencioso, pela paciência que teve comigo durante toda a elaboração deste trabalho, por respeitar as minhas dificuldades e me incentivar a sempre querer aprender mais.

Um agradecimento especial à minha amiga Amélia, por me apoiar nos momentos difíceis desde a graduação até aqui, por fazer piada até dos problemas e, por sempre me ouvir e dar conselhos, ainda que longe e ocupada com o seu mestrado.

Agradeço à minha amiga Mary pelo apoio e incentivo, pela companhia em diversas disciplinas e horas de estudo.

Ao Everton e ao Duílio por estudarem comigo, pelos e-mails trocados, pelo conhecimento matemático compartilhado e também, por ouvirem pacientemente meus constantes falatórios de assuntos acadêmicos ou não.

Agradeço também aos meus familiares, especialmente às titias Eleneide, Eleni e Eliene.

Agradeço ainda as minhas queridas amigas da vida não acadêmica - Lorraine, Thamires, Joyce, Itnã, Jaqueline e Jéssica - por fazerem meus dias mais felizes.

Vários professores fizeram parte da minha história e contribuíram para o meu aprendizado. Gostaria de deixar aqui meus agradecimentos às professoras Iole Freitas, Cláudia Cueva, Rosa Chaves e Lucia Satie, pois além de me ensinarem matemática, contribuíram muito para que eu me tornasse uma professora melhor.

Agradeço ao CNPq pelo apoio financeiro. 


\section{Resumo}

BARROS, J. L. C. O Teorema da Aplicação de Riemann: uma prova livre de integração. 2016. 115 f. Dissertação (Mestrado) - Instituto de Matemática e Estatística, Universidade de São Paulo, São Paulo, 2016.

Neste trabalho, seguindo a abordagem de Weierstrass, temos o objetivo de responder a seguinte questão: conhecida a equivalência entre holomorfia e analiticidade no caso complexo, quais propriedades das funções analíticas podem ser obtidas sem assumir tal equivalência? Analisando esta situação, resultados interessantes serão obtidos sem o uso de qualquer teorema de integração complexa e, para alcançar tal objetivo, nossas principais ferramentas serão a teoria de somas não ordenadas de famílias em $\mathbb{C}$ e propriedades do índice de caminhos fechados. Entre os resultados apresentados estão os conhecidos Teorema Fundamental da Álgebra, Lema de Schwarz, Teorema de Montel, Teorema da Série Dupla de Weierstrass, Princípio do Argumento, Teorema de Rouché, Teorema da Fatoração de Weierstrass, Pequeno Teorema de Picard e o Teorema da Aplicação de Riemann.

Palavras-chave: Abordagem Weierstrassiana, Teorema Fundamental da Álgebra, Princípio do Argumento, Teorema da Aplicação de Riemann, Lema de Schwarz. 


\section{Abstract}

BARROS, J. L. C. The Riemann Mapping Theorem: an integration free proof. 2016. 115

f. Dissertação (Mestrado) - Instituto de Matemática e Estatística, Universidade de São Paulo, São Paulo, 2016.

In this work, following the Weierstrass's approach, we aim to answer the following question: knowing the equivalence between holomorphy and analyticity in the complex case, which properties of analytic functions can be obtained without assuming such equivalence? Through analyzing this situation, interesting results will be obtained without employing of any complex integration theorem and in order to achieve this goal, our main tools will be the theory of unordered sums in $\mathbb{C}$ and properties of winding numbers of closed paths. Among the proven results are the well known Fundamental Theorem of Algebra, Schwarz's Lemma, Montel's Theorem, Weierstrass's Double Series Theorem, Argument Principle, Rouché's Theorem, Weierstrass's Factorization Theorem, Picard's Little Theorem and the Riemann's Mapping Theorem.

Keywords: Weierstrassian Approach, Fundamental Theorem of Algebra, Argument Principle, Riemann's Mapping Theorem, Schwarz's Lemma. 


\section{Sumário}

$\begin{array}{ll}\text { Notação } & \text { ix }\end{array}$

Introdução $\quad$ x

1 Resultados iniciais $\quad 1$

1.1 A topologia do plano complexo . . . . . . . . . . . . . . . . . . 1

1.2 O Teorema Fundamental da Álgebra (TFA) . . . . . . . . . . . . . . . . . . . 4

1.3 Uma primitiva para as raízes $n$-ésimas da unidade . . . . . . . . . . . . . . . . . 9

1.4 Versões polinomiais de teoremas clássicos . . . . . . . . . . . . . . . 12

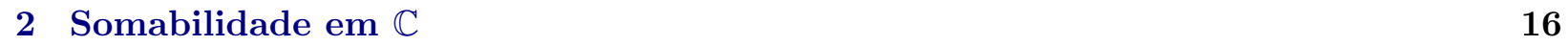

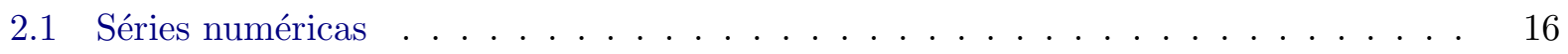

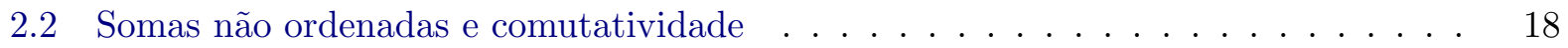

2.3 Somas não ordenadas e associatividade . . . . . . . . . . . . . . . . . . . 21

3 Séries de potências e funções analíticas $\quad 25$

3.1 Séries de potências e suas propriedades . . . . . . . . . . . . . . . . 25

3.2 Funções analíticas . . . . . . . . . . . . . . . . . . . . . . 34

3.2.1 O Teorema da Aplicação Aberta e isomorfismos analíticos . . . . . . . . . . 39

$3.2 .2 \quad$ A Desigualdade de Cauchy . . . . . . . . . . . . . . . . 42

3.2 .3 O Lema de Schwarz . . . . . . . . . . . . . . . . . . . . . . . 45

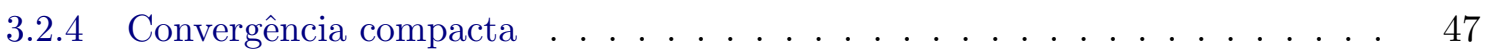

3.2 .5 O Teorema de Montel . . . . . . . . . . . . . . . . . . . . 51

$4 \mathrm{O}$ índice de um caminho fechado e aplicações $\quad 55$

4.1 A exponencial e o logaritmo complexo . . . . . . . . . . . . . . . . . . 55

4.2 O índice de um caminho fechado . . . . . . . . . . . . . . . . . 57

4.3 O Princípio do Argumento e o Teorema de Rouché . . . . . . . . . . . . . . . . . . 64

4.4 O logaritmo de uma função contínua . . . . . . . . . . . . . . . . . . . 67

5 O Teorema da Aplicação de Riemann $\quad 72$

$\begin{array}{lr}\text { A Apêndice } & \mathbf{8 0}\end{array}$

A.1 O Teorema da Fatoração de Weierstrass . . . . . . . . . . . . . . . . . . . 80

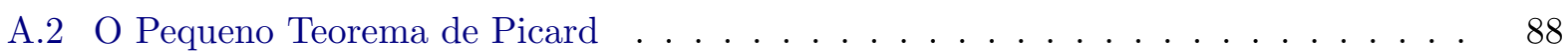

B Apêndice $\quad 95$ 
Referências Bibliográficas 


\title{
Notação
}

\author{
$\mathbb{N} \quad$ o conjunto dos números naturais \\ $\mathbb{N}^{*} \quad$ o conjunto dos números naturais não nulos \\ $\mathbb{Z} \quad$ o conjunto dos números inteiros \\ $\mathbb{Q} \quad$ o conjunto dos números racionais \\ $\mathbb{R} \quad$ o conjunto dos números reais \\ $\mathbb{C} \quad$ o conjunto dos números complexos \\ $\mathbb{C}^{*} \quad$ o conjunto dos números complexos não nulos \\ $B(z ; r)$ bola aberta de centro $z$ e raio $r$ maior que zero \\ $D(z ; r)$ bola fechada de centro $z$ e raio $r$ maior que zero \\ $S^{1} \quad$ conjuntos dos números complexos de norma 1 \\ $\mathbb{D} \quad$ bola aberta de centro 0 e raio 1 \\ $\Omega \quad$ subconjunto aberto de $\mathbb{C}$ \\ $\mathcal{A}(\Omega) \quad$ conjunto das funções analíticas no aberto $\Omega$ com valores em $\mathbb{C}$ \\ $\operatorname{Re}(z) \quad$ parte real do número complexo $z$ \\ $\operatorname{Im}(z) \quad$ parte imaginária do número complexo $z$
}




\section{Introdução}

Nesta dissertação apresentaremos resultados clássicos da Análise Complexa básica via a abordagem de Weierstrass. Isto é, os resultados desenvolvidos serão para funções analíticas no sentido de Weierstrass (funções localmente dadas por séries de potências) e não utilizaremos a equivalência entre holomorfia (uma função definida num aberto é holomorfa se for derivável em todos os pontos do seu domínio) e analiticidade. Isso significa que o nosso tratamento do tema será livre de integração complexa. Entre nossas ferramentas citamos a teoria de somas não ordenadas de famílias em $\mathbb{C}$ e o estudo do índice de caminhos fechados em $\mathbb{C}$.

Optamos por este tema pois, além de nos oferecer ferramentas e métodos de trabalho em Análise Complexa, nos mostra que toda a teoria básica das funções analíticas não necessita de integração complexa para o seu desenvolvimento e, com isto, a abordagem de Weierstrass elimina a falsa impressão de que os conhecidos resultados válidos para funções analíticas somente são verdadeiros porque foram provados para funções holomorfas.

Mostraremos uma versão do Lema de Schwarz mais forte que a usual. Também uma versão mais forte do Teorema de Rouché e do Princípio do Argumento pois, nossa única exigência sobre a curva utilizada é que esta seja contínua.

O trabalho está dividido em cinco capítulos, como segue.

O primeiro capítulo traz resultados básicos de topologia, algumas demonstrações do Teorema Fundamental da Álgebra e, ainda, a prova da existência de uma raiz $n$-ésima primitiva da unidade. Finalizamos este capítulo demonstrando versões polinomiais de resultados da Análise Complexa que serão obtidos com uma técnica semelhante a utilizada nas provas do TFA dadas por O. R. B. de Oliveira ([16]).

O segundo capítulo tem como objetivo introduzir uma ferramenta que será imprescindível para o desenvolvimento do capítulo 3. A teoria de somas não ordenadas dificilmente é encontrada com uma exposição detalhada em textos de Análise Complexa, sendo necessário então discorrer sobre este tópico e demonstrar alguns resultados básicos, porém trabalhando apenas com o essencial a este texto, o corpo dos complexos.

No terceiro capítulo obtemos diversas propriedades das séries de potências - e consequentemente, das funções analíticas - através da teoria de somas não ordenadas de famílias em $\mathbb{C}$. Reunimos neste capítulo uma grande variedade de boas propriedades das funções analíticas. Entre os assuntos tratados estão: Princípios do Módulo Máximo e do Módulo Mínimo, Teorema da Aplicação Aberta, Teorema da Função Inversa, Lema de Schwarz, Representação Local, Teorema da Série Dupla de Weierstrass (Teorema da Convergência de Weierstrass) e o famoso Teorema de Montel. A Desigualdade de Cauchy para as derivadas, usualmente obtida a partir da Fórmula Integral de Cauchy, será também demonstrada numa versão para funções analíticas.

Iniciamos o quarto capítulo com um breve estudo da função exponencial complexa, da sua 
inversa local e de homotopias de caminhos em $\mathbb{C}$. Em seguida, definimos o índice de um caminho fechado com uma abordagem diferente das usuais da área (Análise Complexa), pois não trabalharemos com caminhos de classe $\mathcal{C}^{1}$. Tendo feito toda a parte preliminar, obtemos provas do Princípio do Argumento e do Teorema de Rouché. Ainda mais, enunciamos e provamos uma versão do Teorema de Rouché onde apenas uma das funções é analítica, sendo a outra apenas contínua. Nosso enunciado do Princípio do Argumento é dado a seguir.

Teorema 0.1 (Princípio do Argumento). Sejam $\Omega$ um aberto conexo, $\gamma:[a, b] \rightarrow \Omega$ um caminho fechado homotópico a um ponto de $\Omega$, uma função $f \in \mathcal{A}(\Omega)$ não constante e sem zeros na $\operatorname{Imagem}(\gamma)$. Nestas condições, vale o seguinte

$$
\operatorname{Ind}(f \circ \gamma, 0)=\sum_{a \in Z(f)} \nu(f ; a) \operatorname{Ind}(\gamma ; a)
$$

O último capítulo é sem dúvida um dos pontos centrais deste trabalho, nele apresentamos uma prova do Teorema da Aplicação de Riemann.

Teorema 0.2 (Teorema da Aplicação de Riemann). Se $\Omega$ é um aberto simplesmente conexo não vazio e tal que $\Omega \neq \mathbb{C}$, então $\Omega$ é conformemente equivalente a $\mathbb{D}$.

Este teorema é considerado por muitos como um dos resultados mais importantes da Análise Complexa em uma variável e, como veremos, pode ser provado apenas fazendo uso da teoria das funções analíticas.

No Apêndice A apresentamos uma prova do Teorema da Fatoração de Weierstrass e do Pequeno Teorema de Picard utilizando apenas as ferramentas obtidas ao longo deste trabalho. Como alguns resultados possuem várias demonstrações igualmente importantes, deixamos as variantes para o Apêndice B. 


\section{Capítulo 1}

\section{Resultados iniciais}

Neste capítulo introduzimos alguns conceitos básicos que serão utilizados ao longo deste trabalho e apresentamos demonstrações do Teorema Fundamental da Álgebra (TFA) que não utilizam resultados não elementares da Análise Complexa e uma demonstração elementar da existência de uma raiz $n$-ésima primitiva da unidade sem o uso de teoria dos grupos ou fórmula de Euler. $\mathrm{Na}$ última seção obteremos versões polinomiais de alguns teoremas clássicos.

\subsection{A topologia do plano complexo}

Esta seção estabelece algumas noções básicas da topologia do plano complexo e alguns resultados que serão úteis para o entendimento deste trabalho. Omitiremos as demonstrações, uma vez que são facilmente encontradas (ver [10]).

Como usual, identificamos $\mathbb{R}^{2}$ e $\mathbb{C}$ como espaços vetoriais reais e como espaços topológicos.

Definição 1.1. Dado um número complexo $z=a+$ bi, sua norma é o número real, denotado $|z|$, dado por $|z|=\sqrt{a^{2}+b^{2}}$. Definimos o conjugado de z pelo número complexo $\bar{z}=a-b i$.

Abaixo listamos algumas das propriedades da norma de um número complexo.

Proposição 1.2. Sejam $z, w \in \mathbb{C}$. Valem as seguintes afirmações

(a) $|z w|=|z||w|$.

(b) $|\bar{z}|=|z|$ e $|z|=|-z|$.

(c) $z \bar{z}=|z|^{2}$.

(d) Se $z \neq 0$, então $\left|z^{-1}\right|=|z|^{-1}$.

(d) $\max \{|\operatorname{Re}(z)|,|\operatorname{Im}(z)|\} \leq|z| \leq|\operatorname{Re}(z)|+|\operatorname{Im}(z)|$.

(e) $|z+w| \leq|z|+|w|$.

(f) ||$z|-| w|| \leq|z-w|$.

Decorre do item 1.2 - $(f)$, da proposição anterior, que a função $||:. z \in \mathbb{C} \mapsto|z| \in \mathbb{R}$ é contínua.

Definiremos a seguir alguns conceitos essenciais à compreensão deste texto.

Dados um ponto $z \in \mathbb{C}$ e $r>0$, usaremos a seguinte notação

$$
\begin{aligned}
& B(z ; r)=\{p \in \mathbb{C}:|z-p|<r\} \\
& D(z ; r)=\{p \in \mathbb{C}:|z-p| \leq r\}
\end{aligned}
$$


Definição 1.3. Sejam $U$ um subconjunto de $\mathbb{C} e z \in \mathbb{C}$.

(i) z é ponto interior a $U$ se existe $r>0$ tal que $B(z ; r) \subset U$.

$O$ conjunto dos pontos interiores a $U$ é chamado de interior de $U$.

(ii) z é ponto de aderência de $U$ se para todo $r>0$, temos $B(z ; r) \cap U \neq \emptyset$.

$O$ conjunto dos pontos de aderência de $U$, denotado por $\bar{U}$, é chamado de fecho de $U$.

(iii) z é ponto de acumulação de $U$ se para todo $r>0$, temos $(B(z ; r) \backslash\{z\}) \cap U \neq \emptyset$.

$O$ conjunto dos pontos de acumulação de $U$, denotado por $U^{\prime}$, é chamado de derivado de $U$.

(iv) z é um ponto de fronteira de $U$ se para todo $r>0$, temos $B(z ; r) \cap U \neq \emptyset$ e $B(z ; r) \cap(\mathbb{C} \backslash U) \neq \emptyset$.

$O$ conjunto dos pontos de fronteira de $U$, denotado por $\partial U$, é chamado de fronteira de $U$.

(v) z é um ponto isolado de $U$ se existe $r>0$ tal que $B(z ; r) \cap U=\{z\}$.

(vi) Dizemos que $U$ é aberto em $\mathbb{C}$ se todos os seus pontos são interiores.

(vii) Dizemos que $U$ é fechado em $\mathbb{C}$ se $\mathbb{C} \backslash U$ é aberto.

(viii) Dizemos que U é discreto se todos os seus pontos são isolados.

Proposição 1.4. Seja $U \subset \mathbb{C}$. Valem as seguintes afirmações.

(a) Um conjunto $U$ é fechado se e somente se temos $U=\bar{U}$.

(b) $\bar{U}=U \cup U^{\prime}=U \cup \partial U$.

(c) Um conjunto $U$ é fechado se e somente se contém todos os seus pontos de acumulação.

Demonstração. Ver [10] - p. 12.

Definição 1.5. Sejam $U \subset \mathbb{C} e A \subset U$.

(i) Dizemos que $A$ é aberto em $U$ se existe $V$ aberto de $\mathbb{C}$ tal que $A=V \cap U$. Ainda, A é fechado em $U$ se $U \backslash A$ é aberto em $U$.

(ii) O conjunto $\bar{A} \cap U$ é dito o fecho de $A$ em $U$.

(iii) Um ponto $z \in \mathbb{C}$ é dito ponto de acumulação de $A$ em $U$ se para todo $r>0$ temos $((B(z ; r) \backslash\{z\}) \cap U) \cap$ $A \neq \emptyset$.

Sem risco de confusão, quando dissermos que um conjunto $U$ é aberto (fechado), isto indica que tal conjunto é aberto (fechado) em $\mathbb{C}$.

Uma coleção $\mathcal{A}$ de abertos de $\mathbb{C}$ tal que $U \subset \bigcup_{O \in \mathcal{A}} O$ é dito um recobrimento aberto de $U$. Qualquer subconjunto $\mathcal{A}^{\prime} \subset \mathcal{A}$ para o qual vale $U \subset \bigcup_{O \in \mathcal{A}^{\prime}} O$ é dito um sub-recobrimento de $\mathcal{A}$.

Definição 1.6. Um conjunto $U \subset \mathbb{C}$ é conexo se seus únicos subconjuntos simultaneamente abertos e fechados (em $U$ ) são o vazio e o próprio $U$.

Um conjunto $K \subset \mathbb{C}$ é compacto se todo recobrimento aberto de $K$ admite subrecobrimento finito. 
Dado $A \subset \mathbb{C}$, o conjunto $\mathcal{C} \subset A$ é dito uma componente conexa de $A$, se $\mathcal{C}$ é um conexo maximal contido em $A$. Isto é, $\mathcal{C}$ é conexo e, se $\mathcal{C} \subset \mathcal{C}^{\prime} \subset A$ e $\mathcal{C}^{\prime}$ é conexo, então $\mathcal{C}^{\prime}=\mathcal{C}$. Ainda, se $p \in A$ e

$$
\mathcal{B}=\{U \subset A: p \in U \text { e } U \text { é conexo }\}
$$

definimos a componente conexa de $p(\operatorname{em} A)$ por $\mathcal{C}(p, A)=\bigcup_{U \in \mathcal{B}} U$. Não é difícil ver que para cada $p \in A$, o conjunto $\mathcal{C}(p, A)$ é uma componente conexa de $A$. Ainda mais, se $A$ for aberto então suas componentes conexas são abertas.

A caracterização dos compactos do plano, dada a seguir, será bastante útil.

Teorema 1.7. Seja $K \subset \mathbb{C}$. São equivalentes as afirmações abaixo.

(a) $K$ é compacto.

(b) $K$ é fechado e limitado.

(c) Toda sequência em $K$ admite subsequência convergente à um ponto de $K$.

(d) Todo subconjunto infinito de $K$ tem ponto de acumulação em $K$.

Demonstração. Ver [10] - p. 15.

Teorema 1.8. Sejam $K$ um subconjunto de $\mathbb{C}$ e $f: K \rightarrow \mathbb{C}$ contínua.

(a) Se $K$ for compacto, então $f(K)$ é compacto.

(b) Se $K$ for conexo, então $f(K)$ é conexo.

Demonstração. Ver [10] - p. 19, 25.

Teorema 1.9 (Bolzano - Weierstrass). Sejam $K$ um compacto de $\mathbb{C}$ e $f: K \rightarrow \mathbb{R}$ contínua. Então, $f$ assume máximo e mínimo.

Demonstração. Decorre da equivalência entre $(a)$ e $(b)$ do teorema 1.7 e do item $(a)$ do teorema 1.8 .

Definição 1.10. Sejam $z \in \mathbb{C}$ e dois subconjuntos não vazios, $A$ e $B$, de $\mathbb{C}$.

(i) A distância entre z e A é dada por

$$
d(z, A)=\inf \{|z-a|: a \in A\} .
$$

(ii) A distância entre $A$ e $B$ é dada por

$$
d(A, B)=\inf \{|a-b|: a \in A, b \in B\} .
$$

Observemos que $d(A, B)=\inf \{d(a, B): a \in A\}=\inf \{d(b, A): b \in B\}$.

O item $(d)$ da proposição 1.11 será útil em nossa demonstração do Teorema de Montel. 
Proposição 1.11. Sejam $p, q \in \mathbb{C}$ e $A$ e $B$ subconjuntos não vazios de $\mathbb{C}$. Valem as afirmações a seguir.

(a) $|d(p, A)-d(q, A)| \leq d(p, q)$.

(b) A função $d: z \in \mathbb{C} \mapsto d(z, A)$ é contínua.

(c) $\operatorname{Se} d(p, A)=0$, então $p \in \bar{A}$.

(d) Se $A$ é um compacto, $B$ é fechado e $d(A, B)=0$, então $A \cap B \neq \emptyset$.

\subsection{O Teorema Fundamental da Álgebra (TFA)}

Existem diversas provas do Teorema Fundamental da Álgebra (TFA), a maioria delas utiliza ferramentas que não consideramos elementares. Algumas dessas provas são de fácil compreensão, no entanto, utilizam conceitos nada simples.

Apresentamos duas provas devidas à O. R. B. de Oliveira. A primeira pode ser vista em [16] e está também presente nos livros [25] e [20] e, faz uso da existência de raízes quadradas de números complexos e a segunda é uma variação da apresentada em [17] e faz uso da existência de raízes nésimas de números complexos. A existência de tais raízes será provada e nossas provas do TFA são diretas. Outras demonstrações do TFA surgem a partir da combinação das anteriormente citadas. Utilizando a exponencial complexa é possível demonstrar o TFA de forma não elementar.

No que segue, uma função $p: \mathbb{C} \rightarrow \mathbb{C}$ satisfazendo

$$
p(z)=\sum_{j=0}^{n} a_{j} z^{j}, \text { para todo } z \in \mathbb{C},
$$

onde $a_{j}$ são números complexos fixados, onde $j=0,1, \ldots, n$, com $n \geq 0$ e $a_{n} \neq 0$, é dita um polinômio complexo de grau $n$. Se $p \equiv a_{0}$, com $a_{0} \in \mathbb{C}$, dizemos que $p$ é um polinômio constante.

O TFA garante que todo polinômio complexo e não constante tem uma raiz complexa.

Para provarmos a existência de raízes quadradas de números complexos necessitaremos da existência de raízes quadradas de números reais positivos.

Relembremos que o conjunto dos números reais satisfaz o Axioma do Supremo

Todo subconjunto não vazio de $\mathbb{R}$ e limitado superiormente tem supremo.

Como consequência temos a proposição a seguir.

Proposição 1.12. (a) O conjunto dos números naturais não é limitado superiormente.

(b) Dado $x>0$, existe $n \in \mathbb{N}$ tal que $0<\frac{1}{n}<x$.

Demonstração. O item (a) decorre do Axioma do Supremo. O item (b) é consequência do item $(a)$.

Aplicaremos a proposição anterior para provarmos que todo real positivo tem uma raiz quadrada.

Lema 1.13. Dado $x \geq 0$, existe $y_{0} \geq 0$ tal que $y_{0}^{2}=x$.

Demonstração. Se $x=0$, é claro que $y_{0}=0$. Caso $x>0$, consideremos o conjunto

$$
A=\left\{y \in \mathbb{R}: y>0 \text { e } y^{2} \leq x\right\}
$$


Como $\mathbb{N}$ é ilimitado, existe $a \in \mathbb{N}$ tal que $x<a \leq a^{2}$. Donde segue $y \leq a$, para todo $y \in A$. Ainda, $A$ é não vazio e portanto admite supremo.

Seja $y_{0}=\sup A$ ( $y_{0}$ é positivo).

Afirmação 1. O número real $y_{0}$ satisfaz $y_{0}^{2} \geq x$.

Suponhamos que $y_{0}^{2}<x$. Pelo item $(b)$ da proposição 1.12 , existe $m \in \mathbb{N}$ tal que

$$
0<\frac{1}{m}\left(2 y_{0}+1\right)<x-y_{0}^{2}
$$

Para tal $m$ temos

$$
\left(y_{0}+\frac{1}{m}\right)^{2}=y_{0}^{2}+\frac{2 y_{0}}{m}+\frac{1}{m^{2}}<y_{0}^{2}+\left(x-y_{0}^{2}\right)=x .
$$

Logo, $y_{0}+1 / m \in A$. Mas isto é uma contradição, pois $y_{0}$ é o supremo do conjunto $A$.

Afirmação 2. O número real $y_{0}$ satisfaz $y_{0}^{2} \leq x$.

Suponhamos que $y_{0}^{2}>x$. Nesse caso, existe um natural $m$ tal que

$$
0<\frac{2 y_{0}}{m}<y_{0}^{2}-x
$$

Podemos ainda considerar $1 / m<y_{0}$.

Observemos que se $y_{1}>0$ e $x<y_{1}^{2}$, então $y_{1}$ é cota superior de $A$.

Para finalizarmos, notemos que

$$
\left(y_{0}-\frac{1}{m}\right)^{2}=y_{0}^{2}-\frac{2 y_{0}}{m}+\frac{1}{m^{2}} \geq y_{0}^{2}-\frac{2 y_{0}}{m}>y_{0}^{2}-\left(y_{0}^{2}-x\right)=x .
$$

Segue então que $y_{0}-1 / m$ é cota superior de $A$, o que é uma contradição, pois $y_{0}$ é o supremo do conjunto $A$.

Portanto, $y_{0}^{2}=x$.

Mostraremos a seguir a existência de raízes quadradas de números complexos.

Lema 1.14. Dado $z=a+$ bi complexo não nulo, existe $\zeta \in \mathbb{C}$ tal que $\zeta^{2}=a+b i$.

Demonstração. Seja $\zeta=x+y i$. Logo, $\zeta^{2}=x^{2}-y^{2}+2 x y i$.

Resolvendo o sistema

$$
\left\{\begin{array}{c}
x^{2}-y^{2}=a \\
2 x y=b
\end{array}\right.
$$

encontramos

$$
x^{2}=\frac{a+\sqrt{a^{2}+b^{2}}}{2} \text { e } y^{2}=\frac{-a+\sqrt{a^{2}+b^{2}}}{2} .
$$

Definindo, $\operatorname{sgn}(b)=1$, se $b \geq 0$ e $\operatorname{sgn}(b)=-1$, se $b<0$, obtemos as seguintes soluções para a 
equação $\zeta^{2}=a+b i$

$$
\zeta= \pm\left(\sqrt{\frac{a}{2}+\frac{\sqrt{a^{2}+b^{2}}}{2}}+i \operatorname{sgn}(b) \sqrt{\frac{-a}{2}+\frac{\sqrt{a^{2}+b^{2}}}{2}}\right) .
$$

Dado um polinômio $P$, a função que a cada $z \in \mathbb{C}$ associa o valor $|P(z)|$ será denotada por $|P|$. Ainda mais, assumiremos a continuidade dos polinômios e o algoritmo de Euclides para a divisão de polinômios.

Nossa primeira prova do TFA é dada a seguir.

Utilizaremos a notação $S^{1}=\{\omega \in \mathbb{C}:|\omega|=1\}$.

Teorema 1.15 (TFA). Seja $p(z)=\sum_{j=0}^{n} a_{j} z^{j}$ um polinômio complexo não constante. Então, existe $z_{0} \in \mathbb{C}$ tal que $p\left(z_{0}\right)=0$.

Demonstração 1. Diviremos a prova em duas partes.

Passo 1. Não é difícil verificar que $\lim _{|z| \rightarrow \infty}|p(z)|=\infty$. De fato, como $\lim _{|z| \rightarrow \infty}|z|^{n}=\infty$ e, se $z \neq 0$ temos

$|p(z)| \geq\left|a_{n} z^{n}\right|-\left|a_{n-1} z^{n-1}\right|-\cdots-\left|a_{1} z\right|-\left|a_{0}\right|=\left|a_{n}\right||z|^{n}\left(1-\frac{\left|a_{n-1}\right|}{\left|a_{n}\right||z|}-\cdots-\frac{\left|a_{1}\right|}{\left|a_{n}\right||z|^{n-1}}-\frac{\left|a_{0}\right|}{\left|a_{n}\right||z|^{n}}\right)$.

Seja $R>0$ tal que $|p(z)|>|p(0)|$ se $|z|>R$. Pela continuidade de $|p|$, existe $z_{0} \in \mathbb{C}$ tal que $|p(z)| \geq\left|p\left(z_{0}\right)\right|$ no compacto $D(0 ; R)$. Logo, $\left|p\left(z_{0}\right)\right| \leq|p(0)|$. Consequentemente, $|p(z)| \geq\left|p\left(z_{0}\right)\right|$ para todo $z \in \mathbb{C}$ e $|p|$ tem um mínimo global em $z_{0}$.

Notemos que podemos assumir, sem perda de generalidade, que $z_{0}=0$, uma vez que

$$
\min _{z \in \mathbb{C}}|p(z)|=\min _{z \in \mathbb{C}}\left|p\left(z+z_{0}\right)\right|
$$

$p\left(z+z_{0}\right)$ é um polinômio de grau $n$ e termo independente $p\left(z_{0}\right)$.

Passo 2. Sendo $p$ não constante, existe o menor natural $k \geq 1$ tal que $a_{k} \neq 0$. Obtemos assim que

$$
p(z)=p(0)+z^{k} q(z), \text { com } q \text { polinômio tal que } q(0)=a_{k} \neq 0 \text {. }
$$

Lembremos que $z \bar{z}=|z|^{2}$, se $z \in \mathbb{C}$. Fazendo a substituição $z=r \omega$, com $r>0$ e $\omega \in S^{1}$, obtemos a desigualdade

$$
|p(z)|^{2}-|p(0)|^{2}=2 r^{k} \operatorname{Re}\left[\overline{p(0)} q(r \omega) \omega^{k}\right]+r^{2 k}|q(r \omega)|^{2} \geq 0 .
$$

Fixando $\omega \in S^{1}$ e cancelando $r^{k}$, por continuidade, temos

$$
2 \operatorname{Re}\left[\overline{p(0)} q(0) \omega^{k}\right] \geq 0, \text { para todo } \omega \in S^{1} .
$$


Escrevamos $k=2^{j} m$, com $m$ ímpar e $j \in \mathbb{N}$. Aplicando o lema $1.14 j$ - vezes, escolhemos valores de $\omega \in S^{1}$ tais que $\omega^{2^{j}} \in\{ \pm 1, \pm i\}$. Decorre assim que $\overline{p(0)} q(0)=0$, mas $q(0) \neq 0$ e segue $p(0)=0$.

Na segunda demonstração do TFA, como ferramenta alternativa, utilizamos a norma $|\cdot|_{1}$, dada por

$$
|z|_{1}=|\operatorname{Rez}|+|\operatorname{Imz}|, \text { para todo } z \in \mathbb{C},
$$

e também a existência de raízes $n$-ésimas de números complexos. O lema 1.17 a seguir prova a existência de tais raízes. Uma prova do TFA que não faz uso da existência de raízes $n$-ésimas de números complexos pode ser encontrada em [17].

A proposição a seguir estabelece algumas propriedades da norma $|\cdot|_{1}$ que nos serão úteis.

Proposição 1.16. Dados $z, w \in \mathbb{C}$, valem as desigualdades

$$
\frac{|z|_{1}|w|_{1}}{2} \leq|z w|_{1} \leq|z|_{1}|w|_{1}
$$

Demonstração. Escrevendo $z=a+i b$ e $w=c+i d$, basta notarmos que

$$
\begin{gathered}
(|a|+|b|)^{2}(|c|+|d|)^{2} \leq 2\left(a^{2}+b^{2}\right) 2\left(c^{2}+d^{2}\right)=4\left[(a c-b d)^{2}+(a d+b c)^{2}\right] \leq 4(|a c-b d|+|a d+b c|)^{2} \\
\text { e }|a c-b d|+|a d+b c| \leq|a||c|+|b||d|+|a||d|+|b||c|=(|a|+|b|)(|c|+|d|) .
\end{gathered}
$$

Observemos ainda que, dados $z \in \mathbb{C}$ e $m \in \mathbb{N}$, temos

$$
\begin{gathered}
|z|_{1}^{2} \leq 2 z \bar{z} \mathrm{e} \\
|z|_{1}^{m} \geq\left|z^{m}\right|_{1}=\left|z z^{m-1}\right|_{1} \geq \frac{|z|_{1}\left|z^{m-1}\right|_{1}}{2} \geq \cdots \geq \frac{|z|_{1}^{m}}{2^{m-1}} .
\end{gathered}
$$

Lema 1.17. Dados $n \in \mathbb{N}^{*}$ e $c \in \mathbb{C}^{*}$, existe um número complexo $z_{0}$ tal que $z_{0}^{n}=c$.

Demonstração. Inicialmente observemos que se $P(z)=\sum_{j=0}^{m} a_{j} z^{j}$ é um polinômio não constante e arbitrário, com $a_{m} \neq 0$, então

$$
P(z) \overline{P(z)}=\sum_{j=0}^{m} a_{j} \overline{a_{j}} z^{j} \bar{z}^{j}+2 \sum_{0 \leq j<k \leq m} \operatorname{Re}\left[a_{j} \overline{a_{k}} z^{j} \bar{z}^{k}\right] \geq \frac{\left|a_{m}\right|_{1}^{2}|z|_{1}^{2 m}}{2^{2 m+1}}-\sum_{0 \leq j<k \leq m} 2\left|a_{j}\right|_{1}\left|a_{k}\right|_{1}|z|_{1}^{j+k} .
$$

Portanto, $\lim _{|z|_{1} \rightarrow \infty} P(z) \overline{P(z)}=\infty$.

Seja $R>0$ tal que $P(z) \bar{P}(z)>P(0) \overline{P(0)}$ se $|z|>R$.

Pela continuidade da função $P \bar{P}$, existe $z_{0} \in \mathbb{C}$ tal que

$$
P(z) \bar{P}(z) \geq P\left(z_{0}\right) \overline{P\left(z_{0}\right)}
$$

no compacto $D(0 ; R)$. 
Consequentemente,

$$
P(z) \overline{P(z)} \geq P\left(z_{0}\right) \overline{P\left(z_{0}\right)} \text {, para todo } z \in \mathbb{C} .
$$

A seguir consideremos o polinômio

$$
p(z)=z^{n}-c .
$$

É suficiente provarmos a existência de $z_{0} \in \mathbb{C}$ tal que $p\left(z_{0}\right)=0$. Analisemos dois casos.

Primeiro caso. Suponhamos $n=2$ ou ímpar.

Pelo que foi feito acima, existe $z_{0} \in \mathbb{C}$ tal que $p\left(r \zeta+z_{0}\right) \overline{p\left(r \zeta+z_{0}\right)} \geq p\left(z_{0}\right) \overline{p\left(z_{0}\right)}$, para todos $r>0$ e $\zeta \in \mathbb{C}$.

Afirmamos que $z_{0} \neq 0$. Caso contrário, para todos $r>0$ e $\zeta \in \mathbb{C}$, temos

$$
p(r \zeta \overline{p(r \zeta)} \geq p(0) \overline{p(0)}
$$

Logo,

$$
r^{2 n} \zeta^{n} \bar{\zeta}^{n}-2 r^{n} \operatorname{Re}\left(\zeta^{n} \bar{c}\right) \geq 0 .
$$

Dividindo a expressão anterior por $r^{n}$ e fazendo $r \rightarrow 0^{+}$, tem-se que

$$
2 \operatorname{Re}\left(\zeta^{n} \bar{c}\right) \leq 0, \text { para todo } \zeta \in \mathbb{C}
$$

Se $n=2$, substituindo $\zeta=1, i$ e $\zeta=1 \pm i$, obtemos $c=0$.

Se $n$ é ímpar, substituindo $\zeta= \pm 1$ e $\zeta= \pm i$, também temos $c=0$.

Por hipótese, $c \neq 0$, donde segue que $z_{0} \neq 0$.

Mostraremos a seguir que $p\left(z_{0}\right)=0$.

Utilizando a expressão 1.1, escrevemos $p\left(z+z_{0}\right)=\left(z+z_{0}\right)^{n}-c=p\left(z_{0}\right)+z q(z)$ com $q$ um polinômio complexo e $q(0)=n z_{0}^{n-1} \neq 0$.

Logo,

$$
z \bar{z} q(z) \overline{q(z)}+2 \operatorname{Re}\left[\overline{p\left(z_{0}\right)} z q(z)\right] \geq 0, \text { para todo } z \in \mathbb{C} .
$$

Substituindo $z=r \zeta$ na expressão 1.2 , com $r>0$ e $\zeta \in \mathbb{C}$, por continuidade, obtemos

$$
2 \operatorname{Re}\left[\overline{p\left(z_{0}\right)} q(0) \zeta\right] \geq 0, \text { para todo } \zeta \in \mathbb{C}
$$

Escolhendo $\zeta= \pm 1$ e $\zeta= \pm i$, obtemos que $p\left(z_{0}\right)=0$. Isto é,

$$
z_{0}^{n}=c
$$

Segundo caso. Suponhamos $n$ par e $n \geq 4$.

Escrevendo $n=2^{k} m$, com $k \in \mathbb{N}^{*}$ e $m$ ímpar, segue do primeiro caso que existe $z_{1} \in \mathbb{C}$ tal que $z_{1}^{2}=c$. Ainda, existem $z_{j} \in \mathbb{C}$ tais que $z_{j}^{2}=z_{j-1}$, para $j=2, \ldots, k$, e existe $z_{0} \in \mathbb{C}$ tal que $z_{0}^{m}=z_{k}$. Portanto, $z_{0}^{n}=c$. 
Obteremos a seguir outra demonstração para o TFA.

Teorema 1.18 (TFA). Seja $p(z)=\sum_{j=0}^{n} a_{j} z^{j}$ um polinômio complexo não constante. Então, existe $z_{0} \in \mathbb{C}$ tal que $p\left(z_{0}\right)=0$.

Demonstração 2. Pelo que fizemos anteriormente, existe $z_{0} \in \mathbb{C}$ tal que $p(z) \overline{p(z)} \geq p\left(z_{0}\right) \overline{p\left(z_{0}\right)}$, para todo $z \in \mathbb{C}$. Consideremos a função $q(z)=p\left(z+z_{0}\right)$, então

$$
q(z) \overline{q(z)} \geq q(0) \overline{q(0)} \text {, para todo } z \in \mathbb{C} .
$$

Como $q$ é um polinômio não constante, podemos escrever $q(z)=q(0)+z^{k} h(z)$, com $k \geq 1$ e $h$ um polinômio complexo tal que $h(0) \neq 0$. Segue então que

$$
z^{k} \bar{z}^{k} h(z) \overline{h(z)}+2 \operatorname{Re}\left[\overline{q(0)} z^{k} h(z)\right] \geq 0, \text { para todo } z \in \mathbb{C} .
$$

Substituindo $z=r \zeta$ na expressão 1.3 , com $r>0$ e $\zeta \in \mathbb{C}$, temos

$$
\zeta^{k} r^{k} \bar{\zeta}^{k} r^{k} h(r \zeta) \overline{h(r \zeta)}+2 r^{k} \operatorname{Re}\left[\overline{q(0)} \zeta^{k} h(r \zeta)\right] \geq 0 .
$$

Cancelando $r^{k}$ e fixando $\zeta \in \mathbb{C}$, por argumento de continuidade, obtemos

$$
\operatorname{Re}\left[\overline{q(0)} h(0) \zeta^{k}\right] \geq 0
$$

Pela lema 1.17, podemos escolher $\zeta \in \mathbb{C}$ tais que $\zeta^{k} \in\{ \pm 1, \pm i\}$. Portanto, $\overline{q(0)} h(0)=0$, com $h(0) \neq 0$. Logo, $p\left(z_{0}\right)=q(0)=0$.

Enfatizamos que as provas apresentadas foram simples e diretas. Em [12] vemos a enorme dificuldade em se obter uma demonstração elementar para o TFA. Em 1702, Leibniz tentou provar que o TFA era falso. Posteriormente, Goldbach e Bernoulli cometeram o mesmo erro. A primeira prova considerada correta (mas não era correta) deste teorema é devida a Gauss em 1799, porém era uma versão para polinômios com coeficientes reais. Uma das provas mais simples, além da aqui apresentada, se deve a Argand e foi dada em 1814, porém, não era uma prova direta (enunciada para polinômios complexos).

\subsection{Uma primitiva para as raízes $n$-ésimas da unidade}

O material utilizado para o desenvolvimento desta seção encontra-se em [15].

Dado $n \in \mathbb{N}^{*}$, segue do TFA que a equação $z^{n}=1$ tem $n$ soluções. Ainda mais, tais soluções são distintas. Com efeito, se $\omega^{n}=1$, temos que

$$
z^{n}-1=z^{n}-\omega^{n}=(z-\omega) \sum_{k=0}^{n-1} z^{n-1-k} \omega^{k} .
$$


Escrevendo $P(z)=\sum_{k=0}^{n-1} z^{n-1-k} \omega^{k}$, segue que $P(\omega)=n \omega^{n-1} \neq 0$. Logo, $\omega$ é raiz simples da equação $z^{n}=1$.

Sejam $n \in \mathbb{N}^{*}$ e $\omega$ uma raiz $n$-ésima da unidade. O número $\omega$ é chamado de raiz primitiva das raízes $n$-ésimas da unidade se

$$
1, \omega, \omega^{2}, \ldots, \omega^{n-1}
$$

são todas as $n$ raízes $n$-ésimas da unidade.

Para provarmos a existência de uma raiz primitiva das raízes $n$-ésimas da unidade faremos antes algumas simplificações. Observemos que se $\omega$ é uma raiz primitiva $2 n$-ésima da unidade, então $\omega^{2}, \omega^{4}, \omega^{6}, \ldots, \omega^{2 n}$ são todas as raízes $n$-ésimas da unidade. Logo, podemos supor que $n$ é par. Ainda, como os casos $n=2$ e $n=4$ são triviais, podemos supor $n \geq 6$.

Observemos também que se $\omega$ é uma solução de $z^{n}=1$ então $\pm \omega$ e $\pm \bar{\omega}$ são soluções de $z^{n}=1$. Logo, existe uma solução com partes real e imaginária estritamente positivas. Com efeito, alguns dos números $\pm \omega$ e $\pm \bar{\omega}$ tem partes real e imaginária estritamente positivas.

Consideremos então $\zeta=a+i b$, com $0<a, b<1$, e satisfazendo

$$
0<|\zeta-1|=r=\min \left\{|\omega-1|: \omega^{n}=1 \text { e } \operatorname{Im}(\omega)>0\right\}
$$

No que segue, manteremos as simplificações e notações acima. Ainda, dado $x \in[-1,1]$, denotaremos $z_{x}=x+i \sqrt{1-x^{2}}$.

Provaremos no teorema 1.20 que $\zeta=a+i b$ é uma raiz primitiva das raízes $n$-ésimas da unidade, para isto, determinaremos pontos $x_{0}, x_{1}, \ldots, x_{p} \in[-1,1]$ distintos tais que $z_{x_{k}}=\zeta^{k}$, com $k=0,1, \ldots, p$. O lema 1.19 - (a) será utilizado para obtermos esses pontos distintos.

Lema 1.19. Seja $x \in[-1,1]$. Valem as seguintes afirmações.

(a) A função $\varphi:[-a, 1] \rightarrow[-1, a]$ dada por $\varphi(x)=\operatorname{Re}\left(\zeta z_{x}\right)=a x-b \sqrt{1-x^{2}}$, para todo $x \in[-a, 1]$, é bijetora e estritamente crescente. Sua inversa é $\phi:[-1, a] \rightarrow[-a, 1]$, onde $\phi(y)=\operatorname{Re}\left(\zeta^{-1} z_{y}\right)=$ $a y+b \sqrt{1-y^{2}}$, para todo $y \in[-1, a]$.

(b) Se $x \in[-1,-a] \cup[a, 1]$, então $z_{x}^{n}=1$ se e somente se $x \in\{ \pm 1, \pm a\}$.

Demonstração. Inicialmente vejamos que as funções $\varphi$ e $\phi$ tem suas imagens nos conjuntos $[-1, a]$ e $[-a, 1]$, respectivamente.

Seja $x \in[-a, 1]$. Dado que $\left|\zeta z_{x}\right|=1$, temos $-1 \leq \operatorname{Re}\left(\zeta z_{x}\right)$. Ainda, vale $a x-b \sqrt{1-x^{2}} \leq a$. Com efeito, como $b>0$ temos que $-b \sqrt{1-x^{2}} \leq 0$ e, como $a>0$ e $x \leq 1$, temos $a x \leq a$.

Seja $y \in[-1, a]$. Então, $\operatorname{Re}\left(\zeta^{-1} z_{y}\right) \leq 1$. Ainda, vale $-a \leq a y+b \sqrt{1-x^{2}}$. De fato, como $-1 \leq y$, segue que $-a \leq a y$ e, é claro que $a y \leq a y+b \sqrt{1-x^{2}}$.

Logo,

$$
\varphi([-a, 1]) \subset[-1, a] \quad \text { e } \quad \phi([-1, a]) \subset[-a, 1] .
$$

(a) Se $x \in[-a, 1]$, então $\operatorname{Im}\left(\zeta z_{x}\right)=a \sqrt{1-x^{2}}+b x \geq 0$ (se $x \in[0,1]$, então $-b x \leq 0 \leq a \sqrt{1-x^{2}}$, temos também que a função $h(x)=a \sqrt{1-x^{2}}+b x$ é crescente em $\left.[-a, 0]\right)$. 
Logo, se $y=\varphi(x)$, temos $z_{y}=\zeta z_{x}$ e

$$
\phi(\varphi(x))=\operatorname{Re}\left(\zeta^{-1} z_{y}\right)=\operatorname{Re}\left(\zeta^{-1} \zeta z_{x}\right)=x .
$$

Portanto, $\phi$ é a inversa à esquerda de $\varphi$.

Se $y \in[-1, a]$, temos que $\operatorname{Im}\left(\zeta^{-1} z_{y}\right)=a \sqrt{1-y^{2}}-b y \geq 0$ (a função $g(x)=a \sqrt{1-y^{2}}-b y$ é decrescente em $[0, a]$ e se $y \in[-1,0]$, então $\left.0 \leq-b y \leq a \sqrt{1-y^{2}}-b y\right)$.

Logo, $x=\phi(y)$ satisfaz $z_{x}=\zeta^{-1} z_{y} \mathrm{e}$

$$
\varphi(\phi(y))=\operatorname{Re}\left(\zeta z_{x}\right)=\operatorname{Re}\left(\zeta \zeta^{-1} z_{y}\right)=y
$$

Portanto, $\phi$ é a inversa à direita de $\varphi$. Logo, a função $\varphi$ é bijetora e sua inversa é a função $\phi$.

Observemos que $\varphi$ restrita a $[0,1]$ e $\phi$ restrita a $[-1,0]$ são estritamente crescentes. Ainda, $\phi([-1,0])=[-a, b]$. Logo, $\varphi$ restrita a $[-a, b]$ é estritamente crescente. Portanto $\varphi$ é estritamente crescente em $[-a, 1]$.

(b) Se $x \in\{ \pm a, \pm 1\}$ então $z_{x}^{n}=1$.

Se $x \in(a, 1)$, temos que $\left|z_{x}-1\right|^{2}=2-2 x<2-2 a=r^{2}$. Pela definição de $r$, segue que $z_{x}^{n} \neq 1$.

Dado $x \in(-1,-a)$, pelo caso anterior obtemos

$$
\left(z_{-x}\right)^{n}=\left(-\overline{z_{x}}\right)^{n} \neq 1
$$

Portanto, como $n$ é par segue que $z_{x}^{n} \neq 1$.

O conteúdo do teorema 1.20 é o resultado central desta seção e será obtido sem o uso de ferramentas como a exponencial e teoria dos grupos.

Teorema 1.20. O número $\zeta$ é uma raiz primitiva das raízes n-ésimas da unidade.

Demonstração. Definamos por iteração a sequência $x_{k}=\varphi\left(x_{k-1}\right)$, com $x_{0}=1$ e $k \geq 1$ tal que $x_{k-1} \in[-a, 1]=\operatorname{Dom} \varphi$. Pelo lema 1.19, a função $\varphi$ é estritamente crescente. Logo, $x_{2}=\varphi\left(x_{1}\right)<$ $x_{1}=\varphi\left(x_{0}\right)=a<x_{0}$. Então, por iteração segue

$$
x_{k}<x_{k-1}<\cdots<x_{1}<x_{0} .
$$

Lembremos da demonstração do lema 1.19 que se $y=\varphi(x)$, então $z_{y}=\zeta z_{x}$. Em particular, para $x_{k}=\varphi\left(x_{k-1}\right)$, vale que $z_{x_{k}}=\zeta z_{x_{k-1}}$. Donde por iteração, temos

$$
z_{x_{k}}=\zeta^{k} \text {, se } x_{k-1} \in \operatorname{Dom} \varphi \text { e } z_{x_{0}}=\zeta^{0}=1 .
$$

Uma vez que existem $n$ raízes $n$-ésimas da unidade e os números $z_{x_{j}}$ são raízes $n$-ésimas da unidade, existe o maior $p \in \mathbb{N}$ tal que $x_{p-1} \in \operatorname{Dom} \varphi=[-a, 1]$.

Dado $k=2,3, \ldots, p$, a função $\varphi$ é uma bijeção de $\left[x_{k-1}, x_{k-2}\right]$ sobre $\left[x_{k}, x_{k-1}\right]$. Pelo lema 1.19 - (b), se $x \in\left[x_{1}, x_{0}\right]=[a, 1]$ temos $z_{x}^{n}=1$ se e somente se $x=x_{0}$ ou $x=x_{1}$. Se $y \in\left[x_{2}, x_{1}\right]$ é tal que $z_{y}^{n}=1$, temos que $x=\phi(y)=\operatorname{Re}\left(\zeta^{-1} z_{y}\right) \in\left[x_{1}, x_{0}\right]$ e $z_{x}=\zeta^{-1} z_{y}$. Logo, $z_{x}^{n}=1$, donde segue 
$y=x_{1}$ ou $y=x_{2}$. Utilizando argumento análogo, concluímos que existem somente dois valores de $x$ em $\left[x_{k}, x_{k-1}\right]$ tais que $z_{x}^{n}=1$. A saber,

$$
x=x_{k} \quad \text { e } x=x_{k-1} .
$$

Mostraremos a seguir que $x_{p}=-1$. Pela definição de $p$ temos que $x_{p}=\varphi\left(x_{p-1}\right) \in[-1, a]$, mas $x_{p} \notin \operatorname{Dom} \varphi$. Logo, $x_{p} \in[-1,-a)$. Pelo lema $1.19-(b)$, como $\left(z_{x_{p}}\right)^{n}=1$, obtemos que $x_{p}=-1$ e $\zeta^{p}=-1$.

Os subintervalos $\left[x_{k}, x_{k-1}\right)$, com $k=1,2, \ldots, p$, formam uma partição de $[-1,1)$ e a cada subintervalo corresponde só uma raiz $n$-ésima da unidade no hemisfério

$$
A=\{z \in \mathbb{C}:|z|=1 \text { e } \operatorname{Im}(z) \geq 0\} .
$$

Logo, $\zeta^{0}, \zeta^{1}, \ldots, \zeta^{p}$ são todas as raízes $n$-ésimas da unidade em $A$.

Além disso, se $w$ for uma raiz $n$-ésima da unidade com $\operatorname{Im}(w)<0$, então $\bar{w} \in A$. Logo, existe $k \in\{1,2, \ldots, p-1\}$ tal que $\bar{w}=\zeta^{k}$. Donde segue que $\zeta^{0}, \zeta^{1}, \ldots, \zeta^{p}, \bar{\zeta}, \ldots, \overline{\zeta^{p-1}}$ são todas as $n$ raízes $n$-ésimas da unidade. Logo, $n=2 p$.

Notemos ainda que dado $k=1,2, \ldots, p-1$, temos

$$
\zeta^{p+k} \zeta^{p-k}=\zeta^{2 p}=1 \text { e } \zeta^{p+k}=\left(\zeta^{p-k}\right)^{-1}=\overline{\zeta^{p-k}}
$$

Portanto, as $n$ raízes distintas $n$-ésimas da unidade são

$$
\zeta^{0}=1, \zeta, \ldots, \zeta^{p}=-1, \zeta^{p+1}, \ldots, \zeta^{n-1}
$$

\subsection{Versões polinomiais de teoremas clássicos}

Nesta seção apresentaremos versões polinomiais dos seguintes teoremas: Desigualdade de Gutzmer - Parseval, Princípio do Módulo Máximo, Princípio do Módulo Mínimo e o Teorema da Aplicação Aberta. Nossa principal fonte bibliográfica para o desenvolvimento desta seção é [18]. Não menos importantes são os textos encontrados em [26], [9] e [6].

As demonstrações aqui apresentadas tem suas origens no "método" utilizado na demonstração do TFA. Como interpretado por Caratheodory (p. 109 - [24]), as séries de potências se comportam como polinômios. Poderemos então provar alguns dos resultados a seguir, para funções analíticas, utilizando raciocínio análogo ao empregado na prova do TFA.

A desigualdade a seguir é geralmente demonstrada para funções analíticas complexas como consequência da Identidade de Gutzmer-Parseval e esta por sua vez, obtida a partir da Fórmula Integral de Cauchy.

O nome que damos à desigualdade abaixo segue uma sugestão do professor R. Burckel.

Teorema 1.21 (Desigualdade de Gutzmer-Parseval). Sejam $P(z)=\sum_{j=0}^{n} a_{j} z^{j}$ um polinômio 
complexo e $r>0$. Definamos $M(r)=\max _{|z|=r}|P(z)|$. Então, vale

$$
\sum_{j=0}^{n}\left|a_{j}\right|^{2} r^{2 j} \leq M(r)^{2}
$$

Demonstração. Se $P$ é constante, o resultado é trivial. Suponhamos que $P$ é não constante e para cada $k=0,1, \ldots, 2 n-1$ consideremos os polinômios $P_{k}(z)=P\left(z \omega^{k}\right)$, onde $\omega$ é uma primitiva das raízes $2 n$-ésimas da unidade.

Observando que para um número complexo $z$ vale que $2 \operatorname{Re}(z)=z+\bar{z}$, temos

$$
\left|P_{k}(z)\right|^{2}=\sum_{j=0}^{n}\left|a_{j}\right|^{2}|z|^{2 j}+2 \operatorname{Re} \sum_{0 \leq l<s \leq n}\left[a_{s} \bar{a}_{l} z^{s} \bar{z}^{l} \bar{\omega}^{k l} \omega^{k s}\right] .
$$

Então, como $\bar{\omega}^{k l} \omega^{k s}=\omega^{k(s-l)}$ e $(s-l) \in\{1,2, \ldots, n\}$, se $0 \leq l<s \leq n$, segue que

$$
\sum_{k=0}^{2 n-1} \omega^{k(s-l)}=\frac{1-\omega^{2 n(s-l)}}{1-\omega^{s-l}}=0
$$

Consequentemente, obtemos

$$
\sum_{k=0}^{2 n-1}\left|P_{k}(z)\right|^{2}=2 n \sum_{j=0}^{n}\left|a_{j}\right|^{2}|z|^{2 j}
$$

Finalmente, dado que $\max _{|z|=r}\left|P_{k}(z)\right|=M(r)$, para todo $z \in \mathbb{C}$ com $|z|=r$, temos

$$
\sum_{k=0}^{2 n-1}\left|P_{k}(z)\right|^{2} \leq 2 n M(r)^{2}
$$

Como consequência da Desigualdade de Gutzmer-Parseval, temos uma versão para polinômios da Desigualdade de Cauchy.

Corolário 1.22 (Desigualdade de Cauchy). Sejam $P(z)=\sum_{j=0}^{n} a_{j} z^{j}$ um polinômio complexo e $r>0$. Definamos $M(r)=\max _{|z|=r}|P(z)|$. Então temos

$$
\left|a_{j}\right| \leq \frac{M(r)}{r^{j}}, \text { para todo } j=0,1, \ldots, n .
$$

Mostraremos a seguir o Princípio do Módulo Máximo para polinômios.

Teorema 1.23 (Princípio do Módulo Máximo). Seja $P(z)=\sum_{j=0}^{n} a_{j} z^{j}$ um polinômio complexo não constante. Então, $|P|$ não tem máximo local.

Demonstração. Suponhamos, sem perda de generalidade, que $z_{0}=0$ é ponto de máximo local de $|P|$. 
Seja $R>0$ tal que $|P(z)| \leq|P(0)|$, se $|z|<R$. Logo, a expressão $|P(z)|^{2}-|P(0)|^{2}$ tem sinal constante em $B(0 ; R)$.

Dado que $P$ é não constante, existe $k \geq 1$ tal que

$$
P(z)=P(0)+z^{k} Q(z) \text {, com } Q \text { um polinômio complexo satisfazendo } Q(0) \neq 0 \text {. }
$$

Para $z=r \omega$, com $0<r<R$ e $\omega \in S^{1}$, temos

$$
|P(r \omega)|^{2}-|P(0)|^{2}=2 r^{k} \operatorname{Re}\left[\omega^{k} Q(r \omega) \overline{P(0)}\right]+r^{2 k}|Q(r \omega)|^{2}
$$

Como na prova do TFA, qualquer que seja $\omega \in S^{1}$, temos a expressão de sinal constante

$$
2 \operatorname{Re}\left[\overline{P(0)} Q(0) \omega^{k}\right]
$$

Pela TFA podemos escolher valores de $\omega \in S^{1}$ tais que $\omega^{k} \in\{ \pm 1, \pm i\}$. Resulta então que $\overline{P(0)} Q(0)=0$. Logo, $P \equiv 0$ em $B(0 ; R)$. Dado que $P$ tem no máximo $n$ raízes, temos uma contradição.

A proposição a seguir decorre trivialmente da demonstração do Princípio do Módulo Máximo e então omitiremos a prova.

Teorema 1.24 (Princípio do Módulo Mínimo). Sejam P polinômio complexo não constante e $z_{0}$ um ponto de mínimo local de $|P|$. Então, $P\left(z_{0}\right)=0$.

Dizemos que uma função $f: \Omega \subset \mathbb{C} \rightarrow \mathbb{C}$ é aberta, se para cada aberto $O$ de $\Omega$, o conjunto $f(O)$ é aberto em $\mathbb{C}$.

Provaremos a seguir que um polinômio complexo não constante é uma aplicação aberta.

Teorema 1.25 (Aplicação Aberta). Se P é um polinômio complexo não constante, então $P$ é uma aplicação aberta.

Demonstração. Sejam $O$ subconjunto aberto de $\mathbb{C}$ e $z_{0} \in O$. Como $P$ é não constante, os zeros de $P(z)-P\left(z_{0}\right)$ são isolados. Portanto, existe $r>0$ tal que

$$
D\left(z_{0} ; r\right) \subset O \text { e } P(z)-P\left(z_{0}\right) \neq 0 \text {, se } z \in D\left(z_{0} ; r\right) \backslash\left\{z_{0}\right\} .
$$

Consideremos

$$
R=\min _{z \in \partial D\left(z_{0} ; r\right)} \frac{\left|P(z)-P\left(z_{0}\right)\right|}{2}>0,
$$

um ponto $w \in B\left(P\left(z_{0}\right) ; R\right)$ e a função $P(z)-w$.

Mostraremos que $w \in P(O)$, isto é

$$
B\left(P\left(z_{0}\right) ; R\right) \subset P(O)
$$

Notemos que $\left|P\left(z_{0}\right)-w\right|<R$. Se $z \in \partial D\left(z_{0} ; r\right)$, então

$$
|P(z)-w| \geq\left|P(z)-P\left(z_{0}\right)\right|-\left|P\left(z_{0}\right)-w\right|>2 R-R=R .
$$


Do parágrafo anterior tem-se que o ponto $w_{0}$ de mínimo da função $|P(z)-w|$ no disco fechado $D\left(z_{0} ; r\right)$ está em $B\left(z_{0} ; r\right)$. Como $P(z)-w$ não é constante em $B\left(z_{0} ; r\right)$, pelo Princípio do Módulo Mínimo segue que $P\left(w_{0}\right)-w=0$. Portanto, $P\left(w_{0}\right)=w \operatorname{com} w_{0} \in O$. 


\section{Capítulo 2}

\section{Somabilidade em $\mathbb{C}$}

O objetivo deste capítulo é introduzir a teoria das somas não ordenadas que será utilizada no capítulo 3 para simplificar a demonstração de resultados sobre séries de potências. Iniciaremos com o estudo das séries numéricas, para depois tratarmos de somas não ordenadas de famílias em $\mathbb{C}$. Com esta ferramenta demonstraremos muitas propriedades das séries de potências e, consequentemente, obteremos diversas informações sobre as funções analíticas mesmo sem o conhecimento de que no caso complexo holomorfia e analiticidade são equivalentes.

\subsection{Séries numéricas}

Nesta seção definiremos algumas noções básicas e enunciaremos alguns resultados que podem ser encontrados em [3] e [2].

Definição 2.1. Dada uma sequência $\left(z_{n}\right)$ de números complexos, a série de termo geral $z_{n}$ é a sequência $\left(s_{n}\right)$ definida por

$$
s_{n}=\sum_{j=0}^{n} z_{j}, \text { para todo } n \in \mathbb{N} .
$$

Dizemos que a série é convergente se $\left(s_{n}\right)$ converge. Neste caso, escrevemos $\lim _{n \rightarrow \infty} s_{n}=\sum_{n=0}^{\infty} z_{n}$.

Se a sequência $\left(s_{n}\right)$ não converge, dizemos que a série diverge.

Se $\sum_{n=0}^{\infty}\left|z_{n}\right|<\infty$, a série é dita absolutamente convergente.

É comum denotarmos a série de termo geral $z_{n}$ por $\sum_{n=0}^{\infty} z_{n}$.

Com as conhecidas propriedades operatórias de limites é possível demonstrar o resultado a seguir.

Proposição 2.2. Sejam $\sum_{n=0}^{\infty} z_{n}$ e $\sum_{n=0}^{\infty} w_{n}$ séries convergentes e $\lambda \in \mathbb{C}$.

Então, a série $\sum_{n=0}^{\infty}\left(z_{n}+\lambda w_{n}\right)$ é convergente e satisfaz

$$
\sum_{n=0}^{\infty}\left(z_{n}+\lambda w_{n}\right)=\sum_{n=0}^{\infty} z_{n}+\lambda \sum_{n=0}^{\infty} w_{n}
$$


O seguinte critério permite analisar a convergência (divergência) de uma dada série a partir da convergência (divergência) de outra.

Proposição 2.3 (Critério da comparação para séries). Sejam $\sum_{n=0}^{\infty} z_{n} e \sum_{n=0}^{\infty} w_{n}$ séries complexas. Suponha que existem $c>0$ e $n_{0} \in \mathbb{N}$ tais que $\left|z_{n}\right| \leq c\left|w_{n}\right|$, para todo $n \geq n_{0}$. Se $\sum_{n=0}^{\infty}\left|w_{n}\right|$ converge, então $\sum_{n=0}^{\infty}\left|z_{n}\right|$ também converge.

Observação 2.4. Dado $z \in \mathbb{C}$, temos

$$
\max \{|\operatorname{Re}(z)|,|\operatorname{Im}(z)|\} \leq|z| \leq|\operatorname{Re}(z)|+|\operatorname{Im}(z)|
$$

Observação 2.5. É conhecido que se $\sum_{n=0}^{\infty}\left|z_{n}\right|$ converge, então $\sum_{n=0}^{\infty} z_{n}$ também converge. Ainda, vale a desigualdade

$$
\left|\sum_{n=0}^{\infty} z_{n}\right| \leq \sum_{n=0}^{\infty}\left|z_{n}\right|
$$

Definiremos a seguir noção de valor de aderência e de limite superior de uma sequência.

Definição 2.6. Seja $\left(a_{n}\right)$ uma sequência de números reais. Dizemos que $a \in[-\infty,+\infty]$ é um valor de aderência de $\left(a_{n}\right)$ se existe uma subsequência $\left(a_{n_{k}}\right)_{k \geq 1}$ convergindo ao valor a.

O limite superior da sequência é o valor

$$
\lim \sup a_{n}=\sup \left\{a \in[-\infty,+\infty]: \text { a é valor de aderência de }\left(a_{n}\right)\right\}
$$

Toda sequência em $\mathbb{R}$ e limitada tem uma subsequência convergente e então admite um valor de aderência em $\mathbb{R}$. Se a sequência for ilimitada, então $-\infty$ ou $+\infty$ é um valor de aderência (ou ambos).

Proposição 2.7. Seja $\left(a_{n}\right)$ sequência em $\mathbb{R}$ limitada. Dado $\varepsilon>0$, existe $n_{0} \in \mathbb{N}$ tal que

$$
a_{n} \leq \lim \sup a_{n}+\varepsilon, \text { para todo } n \geq n_{0} .
$$

Para finalizarmos esta seção, enunciamos dois critérios para a convergência (divergência) de uma série em $\mathbb{C}$ que serão utilizados posteriormente.

Teorema 2.8 (Critério da Raiz). Sejam $\sum_{n=0}^{\infty} z_{n}$ uma série complexa e $L=\limsup \sqrt[n]{\left|z_{n}\right|}$. Vale o que segue.

(a) Se $L<1$, a série converge absolutamente.

(b) Se $L>1$, a série diverge.

Teorema 2.9 (Critério da Razão). Seja $\sum_{n=0}^{\infty} z_{n}$ uma série complexa com $z_{n} \neq 0$, para todo $n \in \mathbb{N}$. Suponhamos que existe $L=\lim _{n \rightarrow \infty} \frac{\left|z_{n+1}\right|}{\left|z_{n}\right|}$. Então, vale o seguinte.

(a) Se $L<1$, a série converge absolutamente.

(b) Se $L>1$, a série diverge. 


\subsection{Somas não ordenadas e comutatividade}

Iniciaremos esta seção provando um resultado bastante conhecido para séries de termos positivos e nos basearemos nele para definirmos somabilidade de familias de números complexos. A referência utilizada para esta seção encontra-se em [19].

Teorema 2.10. Se $\left(p_{n}\right)$ é uma sequência em $[0,+\infty]$, então

$$
\sum_{n=0}^{\infty} p_{n}=\rho, \quad \text { onde } \rho=\sup \left\{\sum_{n \in F} p_{n}: F \subset \mathbb{N} \text { e F finito }\right\} .
$$

Demonstração. Sejam $\left(s_{n}\right)$ a sequência das somas parciais da série dada e $F \subset \mathbb{N}, F$ finito e arbitrário. Então, valem as desigualdades

$$
\sum_{j \in F} p_{j} \leq s_{\operatorname{máx}(F)} \leq \sum_{n=0}^{\infty} p_{n} \quad \text { e } \quad s_{n}=\sum_{j=0}^{n} p_{j} \leq \rho .
$$

Decorre assim que

$$
\rho=\sup \left\{\sum_{j \in F} p_{j}: F \subset \mathbb{N} e F \text { finito }\right\} \leq \sum_{n=0}^{\infty} p_{n}=\lim _{n \rightarrow \infty} s_{n} \leq \rho .
$$

Lembremos que uma sequência num conjunto $A$ é uma função $x: \mathbb{N} \rightarrow A$. Denotamos tal função por $\left(x_{n}\right)$ e $x_{n}=x(n)$ é dito o $n$-ésimo termo da sequência.

A definição a seguir generaliza tal conceito.

Definição 2.11. Seja $J$ um conjunto enumerável. Uma familia em $\mathbb{C}$, indexada em $J$, é uma função $x: J \rightarrow \mathbb{C}$, que denotamos por $\left(x_{j}\right)_{J}$ e $x_{j}=x(j)$ é dito o j-ésimo termo da família.

As operações de soma (entre duas famílias) e multiplicação por escalar são as usuais entre funções.

Notemos que toda sequência é uma família, com $J=\mathbb{N}$.

No que segue $J$ e $L$ denotam conjuntos enumeráveis de índices.

Definição 2.12. Seja $\left(a_{j}\right)_{J}$ uma família de números reais. Definimos as partes positiva e negativa de $a_{j}$, indicadas $p_{j}$ e $q_{j}$, respectivamente, por

$$
p_{j}=\left\{\begin{array}{ll}
a_{j}, & \text { se } a_{j} \geq 0 \\
0, & \text { se } a_{j} \leq 0,
\end{array} \quad q_{j}= \begin{cases}0, & \text { se } a_{j} \geq 0 \\
-a_{j}, & \text { se } a_{j} \leq 0\end{cases}\right.
$$

As famílias $\left(p_{j}\right)_{J}$ e $\left(q_{j}\right)_{J}$ são ditas as familias das partes positiva e negativa de $\left(a_{j}\right)_{J}$.

A observação 2.13 decorre da definição acima.

Observação 2.13. Dada $\left(a_{j}\right)_{J}$ família de números reais, não é difícil ver que para todo $j \in J$, temos

$$
\left\{\begin{array} { c } 
{ a _ { j } = p _ { j } - q _ { j } } \\
{ | a _ { j } | = p _ { j } + q _ { j } , }
\end{array} \quad e \quad \left\{\begin{array}{l}
0 \leq p_{j} \leq\left|a_{j}\right| \\
0 \leq q_{j} \leq\left|a_{j}\right|
\end{array}\right.\right.
$$


Definiremos abaixo a noção de somabilidade para famílias de números complexos.

Definição 2.14. (a) Uma família $\left(p_{j}\right)_{J}$ de termos positivos é dita somável se

$$
\sup \left\{\sum_{j \in F} p_{j}: F \subset J \text { e F finito }\right\}<\infty .
$$

Definimos sua soma por

$$
\sum_{J} p_{j}=\sup \left\{\sum_{j \in F} p_{j}: F \subset J \text { e F finito }\right\} .
$$

(b) Uma família de números reais $\left(a_{j}\right)_{J}$ é dita somável se as famílias $\left(p_{j}\right)_{J}$ e $\left(q_{j}\right)_{J}$ são somáveis. Definimos sua soma por

$$
\sum_{J} a_{j}=\sum_{J} p_{j}-\sum_{J} q_{j}
$$

(c) Uma família de números complexos $\left(z_{j}\right)_{J}$ é dita somável se as famílias $\left(\operatorname{Re}\left(z_{j}\right)\right)_{J}$ e $\left(\operatorname{Im}\left(z_{j}\right)\right)_{J}$ são somáveis. Definimos sua soma por

$$
\sum_{J} z_{j}=\sum_{J} \operatorname{Re}\left(z_{j}\right)+i \sum_{J} \operatorname{Im}\left(z_{j}\right)
$$

Uma pergunta natural é: se para verificarmos a somabilidade de uma família de termos positivos analisamos apenas o supremo do conjunto das somas finitas, será que a ordenação do conjunto de índices é importante ? A resposta é não, como mostra o lema abaixo.

Lema 2.15. Se $\sigma: L \rightarrow J$ é uma bijeção e $\left(p_{j}\right)_{j \in J}$ é uma família somável de termos positivos, então

$$
\sum_{j \in J} p_{j}=\sum_{l \in L} p_{\sigma(l)}
$$

Demonstração. É suficiente mostrarmos que

$$
\left\{\sum_{j \in F} p_{j}: F \subset J \text { e F finito }\right\}=\left\{\sum_{l \in \bar{F}} p_{\sigma(l)}: \bar{F} \subset L \text { e } \bar{F} \text { finito }\right\} .
$$

Basta notarmos que dados $F \subset J$ e $\bar{F} \subset L$ finitos, temos

$$
\begin{aligned}
& \sum_{j \in F} p_{j}=\sum_{l \in \sigma^{-1}(F)} p_{\sigma(l)}, \\
& \sum_{l \in \bar{F}} p_{\sigma(l)}=\sum_{j \in \sigma(\bar{F})} p_{j} .
\end{aligned}
$$

O teorema abaixo facilitará nosso trabalho, além de relacionar os conceitos de série e famílias de números complexos. 
Teorema 2.16. Sejam $\left(z_{j}\right)_{J}$ uma família de números complexos e $\sigma: \mathbb{N} \rightarrow J$ bijetora. São equivalentes as afirmações abaixo.

(a) $\left(z_{j}\right)_{J}$ é somável.

(b) $\left(z_{j}\right)_{J}$ é absolutamente somável (isto é, $\left(\left|z_{j}\right|\right)_{J}$ é somável).

(c) Existe $M>0$ tal que

$$
\sum_{F}\left|z_{j}\right| \leq M, \text { para todo } F \subset J, \text { com } F \text { finito. }
$$

(d) $\sum_{n=0}^{\infty}\left|z_{\sigma(n)}\right|$ é convergente.

Valendo uma das afirmações acima, temos a igualdade

$$
\sum_{J} z_{j}=\sum_{n=0}^{\infty} z_{\sigma(n)} .
$$

Demonstração. Consideremos as famílias $\left(\operatorname{Re}\left(z_{j}\right)\right)_{J}$ e $\left(\operatorname{Im}\left(z_{j}\right)\right)_{J}$ e as famílias de suas partes positivas, $\left(p_{j}\right)_{J},\left(P_{j}\right)_{J}$, e as negativas $\left(q_{j}\right)_{J}$ e $\left(Q_{j}\right)_{J}$, respectivamente.

$(a \Leftrightarrow b)$ Utilizando as relações das observações 2.4 e 2.13 temos, para todo $j \in J$

$$
0 \leq \operatorname{máx}\left\{p_{j}, q_{j}, P_{j}, Q_{j}\right\} \leq\left|z_{j}\right| \leq p_{j}+q_{j}+P_{j}+Q_{j} .
$$

Segue que $\sum\left|z_{j}\right|<\infty$ se e somente se $\sum p_{j}, \sum q_{j}, \sum P_{j}$ e $\sum Q_{j}$ são finitas.

Portanto, $\left(\left|z_{j}\right|\right)_{J}$ é somável se e somente se $\left(z_{j}\right)_{J}$ é somável.

$(b \Leftrightarrow c)$ Decorre da definição de somabilidade 2.14.

$(c \Leftrightarrow d)$ Trivial.

Ainda, se vale $(a)$ ou $(b)$ ou $(c)$, pela definição 2.14 , lema 2.15, teorema 2.10 e a proposição 2.2 , segue que

$$
\sum_{J} z_{j}=\sum_{n=0}^{\infty} z_{\sigma(n)} .
$$

Corolário 2.17. Seja $\left(z_{j}\right)_{J}$ uma família somável e $L \subset J$. Então, a família $\left(z_{l}\right)_{L}$ é somável.

Demonstração. Segue do teorema anterior e da desigualdade

$$
\sum_{l \in L}\left|z_{l}\right| \leq \sum_{j \in J}\left|z_{j}\right|
$$

As propriedades provadas no corolário 2.18 serão muito úteis. 
Corolário 2.18. Sejam $\left(z_{j}\right)_{J}$ e $\left(w_{j}\right)_{J}$ famílias somáveis em $\mathbb{C}$ e $\lambda \in \mathbb{C}$. Então as familias $\left(z_{j}+w_{j}\right)_{J}$ $e\left(\lambda z_{j}\right)_{J}$ são somáveis e valem as seguintes propriedades

(a) $\sum_{j \in J}\left(z_{j}+w_{j}\right)=\sum_{j \in J} z_{j}+\sum_{j \in J} w_{j}$.

(b) $\sum_{j \in J}\left(\lambda z_{j}\right)=\lambda \sum_{j \in J} z_{j}$.

(c) $\left|\sum_{j \in J} z_{j}\right| \leq \sum_{j \in J}\left|z_{j}\right|$.

Demonstração. Seja $\sigma: \mathbb{N} \rightarrow J$ uma bijeção.

(a) Para todo $n \in \mathbb{N}$, temos

$$
\left|z_{\sigma(n)}+w_{\sigma(n)}\right| \leq\left|z_{\sigma(n)}\right|+\left|w_{\sigma(n)}\right|
$$

Logo, pelo Critério da Comparação e o teorema 2.16, temos que $\left(z_{j}+w_{j}\right)_{J}$ é somável.

E mais, temos que

$$
\sum_{J}\left(z_{j}+w_{j}\right)=\sum_{n=0}^{\infty}\left(z_{\sigma(n)}+w_{\sigma(n)}\right)=\sum_{n=0}^{\infty} z_{\sigma(n)}+\sum_{n=0}^{\infty} w_{\sigma(n)}=\sum_{J} z_{j}+\sum_{J} w_{j} .
$$

(b) Segue os passos do item $(a)$.

(c) Pela observação 2.5 e o teorema 2.16, temos as desigualdades abaixo

$$
\left|\sum_{j \in J} z_{j}\right|=\left|\sum_{n=0}^{\infty} z_{\sigma(n)}\right| \leq \sum_{n=0}^{\infty}\left|z_{\sigma(n)}\right|=\sum_{j \in J}\left|z_{j}\right|
$$

Corolário 2.19 (Critério da comparação para somas não ordenadas). Sejam $\left(z_{n}\right)_{\mathbb{N}} e\left(w_{n}\right)_{\mathbb{N}}$ sequências em $\mathbb{C}$ tais que $\left|z_{n}\right| \leq\left|w_{n}\right|$, para todo $n \in \mathbb{N}$. Se $\left(w_{n}\right)_{\mathbb{N}}$ é somável, então $\left(z_{n}\right)_{\mathbb{N}}$ é somável.

\subsection{Somas não ordenadas e associatividade}

Os resultados desta seção serão essenciais nas demonstrações de resultados válidos para séries de potências. A bibliografia utilizada é [19].

Definição 2.20. Seja $J$ conjunto enumerável (infinito). Uma partição de $J$ é uma coleção de subconjuntos $\left\{L_{l}: L_{l} \subset J\right.$ e $\left.l \in L\right\}$, onde $L$ é um conjunto de indices, satisfazendo $L_{l} \cap L_{k}=\emptyset$ para quaisquer $l, k \in L$ distintos e $J=\bigcup_{l \in L} L_{l}$.

Denotamos a partição por $\bigcup_{l \in L} L_{l}$.

O teorema 2.21 e o corolário 2.24 são os principais resultados desta seção.

Teorema 2.21. Sejam $\left(p_{j}\right)_{J}$ uma família em $[0,+\infty]$ e $\bigcup_{l \in L} L_{l}$ uma partição arbitrária de J. Então,

$$
\sum_{J} p_{j}=\sum_{l \in L} \sum_{j \in L_{l}} p_{j}
$$


Demonstração. Mostraremos primeiramente que $\sum_{J} p_{j} \leq \sum_{l \in L} \sum_{j \in L_{l}} p_{j}$.

Dado $F \subset J$ finito com $F$ finito, existe um subconjunto finito de $L,\left\{l_{1}, l_{2}, \ldots, l_{m}\right\}\left(l_{j} \neq l_{k}\right.$, se $j \neq k)$ tal que

$$
F \subset L_{l_{1}} \cup L_{l_{2}} \cup \ldots \cup L_{l_{m}}
$$

Logo,

$$
\sum_{j \in F} p_{j} \leq \sum_{j \in L_{l_{1}}} p_{j}+\sum_{j \in L_{l_{2}}} p_{j}+\cdots+\sum_{j \in L_{l_{m}}} p_{j} \leq \sum_{l \in L} \sum_{j \in L_{l}} p_{j} .
$$

Uma vez que $F$ é arbitrário, segue da definição de família somável que

$$
\sum_{J} p_{j} \leq \sum_{l \in L} \sum_{j \in L_{l}} p_{j}
$$

A seguir obteremos a outra desigualdade.

Consideremos $\left\{l_{1}, l_{2}, \ldots, l_{m}\right\} \subset L$ qualquer $\left(l_{j} \neq l_{k}\right.$, se $\left.j \neq k\right)$. Para cada $k \in\{1,2, \ldots, m\}$ seja $F_{k} \subset L_{l_{k}}$, com $F_{k}$ finito e arbitrário. Notemos que os conjuntos $F_{1}, F_{2}, \ldots, F_{m}$ são dois a dois disjuntos.

Portanto,

$$
\sum_{\cup_{k=1}^{m} F_{k}} p_{j}=\sum_{j \in F_{1}} p_{j}+\sum_{j \in F_{2}} p_{j}+\cdots+\sum_{j \in F_{m}} p_{j} \leq \sum_{J} p_{j}
$$

Fixando $F_{2}, \ldots, F_{m}$ e, tomando o supremo sobre a família de todos os conjuntos finitos de $L_{l_{1}}$, temos

$$
\sum_{j \in L_{1}} p_{j}+\sum_{j \in F_{2}} p_{j}+\cdots+\sum_{j \in F_{m}} p_{j} \leq \sum_{J} p_{j}
$$

Procedendo como no páragrafo anterior, obtemos indutivamente

$$
\sum_{j \in L_{l_{1}}} p_{j}+\sum_{j \in L_{j_{2}}} p_{j}+\cdots+\sum_{j \in L_{l_{m}}} p_{j} \leq \sum_{J} p_{j}
$$

Como $\left\{l_{1}, l_{2}, \ldots, l_{m}\right\} \subset L$ é um subconjunto arbitrário, decorre que

$$
\sum_{l \in L} \sum_{j \in L_{l}} p_{j} \leq \sum p_{j}
$$

Para exemplificar o uso das propriedades obtidas até o momento, faremos com excesso de detalhes o seguinte exemplo.

Exemplo 2.22. Consideremos $0<a, b<1$ e a familia $\left(a^{n} b^{m}\right)_{\mathbb{N} \times \mathbb{N}}$. Vamos verificar que tal família é somável e determinar sua soma.

A família $\left(a^{n} b^{m}\right)_{\mathbb{N} \times \mathbb{N}}$ é de termos positivos. Então, pelo teorema 2.21 segue

$$
\sum a^{n} b^{m}=\sum_{n \in \mathbb{N}} \sum_{(n, m), m \in \mathbb{N}} a^{n} b^{m}
$$

Para cada $n \in \mathbb{N}, \sigma: m \in \mathbb{N} \mapsto(n, m) \in \mathbb{N} \times \mathbb{N}$ é uma bijeção, assim, pelo lema 2.15, segue-se 
que

$$
\sum_{(n, m), m \in \mathbb{N}} a^{n} b^{m}=\sum_{m \in \mathbb{N}} a^{n} b^{m}
$$

Mas, utilizando a convergência da série geométrica, pelo corolário 2.18 - (b) e teorema 2.16, respectivamente, temos

$$
\sum_{m \in \mathbb{N}} a^{n} b^{m}=a^{n} \sum_{m \in \mathbb{N}} b^{m}=\frac{a^{n}}{1-b} .
$$

Finalmente, pelos mesmos argumentos do parágrafo anterior, obtemos a igualdade

$$
\sum a^{n} b^{m}=\sum_{n \in \mathbb{N}} \frac{a^{n}}{1-b}=\frac{1}{(1-a)(1-b)} .
$$

Vejamos ainda um outro exemplo.

Exemplo 2.23. Se $k \in \mathbb{N}$, afirmamos: a família em $\mathbb{N}^{*} \times \mathbb{N}^{*}$ dada por $\left(\frac{1}{\left(m^{2}+n^{2}\right)^{k}}\right)$ é somável se e somente se $k \geq 2$.

Suponha $k=1$. Observemos que

$$
m^{2}+n^{2} \leq(m+n)^{2}, \text { para todos } m, n \in \mathbb{N}
$$

Logo, é suficiente mostrarmos que a familia $\left(\frac{1}{(m+n)^{2}}\right)$ não é somável.

Aplicando o teorema 2.21 temos que

$$
\sum \frac{1}{(m+n)^{2}}=\sum_{p \geq 2} \sum_{m+n=p} \frac{1}{(m+n)^{2}}=\sum_{p \geq 2} \frac{p-1}{p^{2}}=\sum_{p \geq 2}\left(\frac{1}{p}-\frac{1}{p^{2}}\right)=\infty .
$$

Suponha $k \geq 2$. Observemos que

$$
\left(m^{2}+n^{2}\right)^{k} \geq m^{2} n^{2} \text { para todos } m, n \in \mathbb{N} .
$$

Mas,

$$
\sum \frac{1}{m^{2} n^{2}}=\sum_{m} \sum_{n} \frac{1}{m^{2} n^{2}}=\left(\sum_{m=1}^{\infty} \frac{1}{m^{2}}\right)\left(\sum_{n=1}^{\infty} \frac{1}{n^{2}}\right)<\infty .
$$

Corolário 2.24 (Lei associativa). Sejam $\left(z_{j}\right)_{J}$ uma sequência somável de números complexos e $\bigcup_{l \in L} L_{l}$ uma partição arbitrária de J. Então a familia $\left(z_{j}\right)_{j \in L_{l}}$ é somável, para todo $l \in L$ e vale

$$
\sum z_{j}=\sum_{l \in L} \sum_{j \in L_{l}} z_{j}
$$

Demonstração. Decorre do corolário 2.17 que a família $\left(z_{j}\right)_{j \in L_{l}}$ é somável, para todo $l \in L$.

Se $\left(z_{j}\right)_{J}$ é uma família de números reais o resultado segue da definição 2.14 - $(a)$, do teorema 2.21 e do corolário $2.18-(a)$.

Se $\left(z_{j}\right)_{J}$ é uma família de números complexos, então o resultado segue da definição 2.14 - $(b)$, do caso anterior e do corolário 2.18 - $(a)$ e $(b)$. 
Encerramos este capítulo com mais alguns exemplos de aplicação dos resultados obtidos, para que os utilizemos com mais facilidade no próximo capítulo.

Definição 2.25. Dadas $\sum_{n=0}^{\infty} z_{n} e \sum_{m=0}^{\infty} w_{m}$ série convergentes, seu produto de Cauchy é a série

$$
\sum_{p=0}^{\infty} c_{p}, \text { onde } c_{p}=\sum_{n+m=p} z_{n} w_{m}
$$

Corolário 2.26. Sejam $\sum_{n=0}^{\infty} z_{n}=z$ e $\sum_{m=0}^{\infty} w_{m}=w$ duas séries absolutamente convergentes. Então, valem as seguintes afirmações.

(a) $\sum\left|z_{n} w_{m}\right|<\infty$

(b) $\sum z_{n} w_{m}=z w$.

(c) Seu produto de Cauchy é uma série absolutamente convergente e

$$
\sum_{p=0}^{\infty}\left(\sum_{n+m=p} z_{n} w_{m}\right)=z w
$$

Demonstração. Do teorema 2.21, corolário 2.18 - (b) e teorema 2.16, deduzimos que

$$
\sum_{(n, m)}\left|z_{n} w_{m}\right|=\sum_{n \in \mathbb{N}} \sum_{m \in \mathbb{N}}\left|z_{n}\right|\left|w_{m}\right|=\left(\sum\left|z_{n}\right|\right)\left(\sum\left|w_{m}\right|\right)<\infty .
$$

(a) Aplicando o corolário 2.24, corolário 2.18 - (b) e teorema 2.16, obtemos as igualdades

$$
\sum z_{n} w_{m}=\sum_{n \in \mathbb{N}} \sum_{m \in \mathbb{N}} z_{n} w_{m}=\left(\sum_{n=0}^{\infty} z_{n}\right)\left(\sum_{m=0}^{\infty} w_{m}\right)=z w .
$$

(b) Pelo item $(a)$, pelo corolário 2.24 com a partição $\bigcup_{p \in \mathbb{N}} L_{p}$ de $\mathbb{N} \times \mathbb{N}$, onde

$$
L_{p}=\{(n, m) \in \mathbb{N} \times \mathbb{N}: n+m=p\},
$$

e pelo teorema 2.16 , temos

$$
z w=\sum z_{n} w_{m}=\sum_{p \in \mathbb{N}}\left(\sum_{n+m=p} z_{n} w_{m}\right)=\sum_{p \in \mathbb{N}} c_{p}=\sum_{p=0}^{\infty} c_{p},
$$

onde $c_{p}=\sum_{n+m=p} z_{n} w_{m}$ e $\sum_{p=0}^{\infty}\left|c_{p}\right|<\infty$. 


\section{Capítulo 3}

\section{Séries de potências e funções analíticas}

Neste capítulo demonstraremos importantes resultados sobre funções analíticas tais como o Teorema da Aplicação Aberta, Princípios do Módulo Máximo e Mínimo, Teorema de Representação Local, Teorema da Função Inversa Local e Global, Desigualdade de Cauchy, Lema de Schwarz, Teorema da Série Dupla de Weierstrass (Teorema da Convergência de Weierstrass) e o Teorema de Montel, utilizando apenas a teoria das séries de potências.

Muitos dos resultados aqui apresentados são geralmente demonstrados a partir da Fórmula Integral de Cauchy ou algum outro teorema sobre integração, nosso objetivo é obtê-los sem o uso de tais ferramentas.

Acreditamos que as técnicas aqui utilizadas sirvam para simplificarmos demonstrações e obtermos resultados que posteriormente possam ser aplicadas para a obtenção de outros resultados relacionados em matemática.

As demonstrações apresentadas neste capítulo foram fortemente baseadas em [19], [18], [8] e [28]. Também utilizamos [7] e [14].

\subsection{Séries de potências e suas propriedades}

Iniciamos com alguns conceitos básicos necessários para o desenvolvimento do capítulo.

Definição 3.1. Dada $\left(f_{n}\right)_{n \geq 0}$ uma sequência de funções definidas em $\Omega$, dizemos que $\left(f_{n}\right)_{n \geq 0}$ converge uniformemente em $\Omega$ à função $f$ se, para todo $\varepsilon>0$, existe $n_{0} \in \mathbb{N}$ tal que

$$
\sup \left\{\left|f_{n}(x)-f(x)\right|: x \in \Omega\right\}<\varepsilon, \text { para todo } n \geq n_{0}
$$

Decorre das definições de continuidade e convergência uniforme a seguinte proposição.

Proposição 3.2. Seja $\left(f_{n}\right)_{n \geq 0}$ uma sequência de funções contínuas definidas em $\Omega$. Se $\left(f_{n}\right)$ converge uniformemente em $\Omega$ à função $f$, então $f$ é contínua.

Definição 3.3. Seja $\left(f_{n}\right)_{n \geq 0}$ uma sequência de funções definidas em $\Omega$. A série (de funções) gerada por $\left(f_{n}\right)_{n \geq 0}$ é a sequência $\left(s_{n}\right)_{n \geq 0}$, dada por $s_{n}=f_{1}+f_{2}+\cdots+f_{n}$, para todo $n \in \mathbb{N}$. 
Dizemos que a série converge (simplesmente) à função $f: \Omega \rightarrow \mathbb{C}$, se $\lim _{n \rightarrow \infty} s_{n}(x)=f(x)$, qualquer que seja $x$ em $\Omega$. Escrevemos $f(x)=\sum_{n=0}^{\infty} f_{n}(x)$, com $x \in \Omega$, para indicar que a série converge à função $f$.

Por simplicidade, denotamos uma série de funções por $\sum_{n=0}^{\infty} f_{n}$ ou $\sum_{n=0}^{\infty} f_{n}(x)$ e chamamos $\left(s_{n}\right)_{n \geq 0}$ de sequência das somas parciais.

Ainda, dizemos que a série $\sum_{n=0}^{\infty} f_{n}$ converge uniformemente à função $f$, se a sua sequência das somas parciais converge uniformemente à $f$. à $f$.

Observemos que se $\sum_{n=0}^{\infty} f_{n}$ converge uniformemente à $f$, então a série converge (simplesmente)

Consideremos $\left(a_{n}\right)_{n \geq 0}$ uma sequência de números complexos e $z_{0} \in \mathbb{C}$ fixado.

Definição 3.4. A série de funções $\sum_{n=0}^{\infty} a_{n}\left(z-z_{0}\right)^{n}$ é chamada de série de potências centrada em $z_{0}$ e coeficientes $\left(a_{n}\right)_{n \geq 0}$.

Dado $w \in \mathbb{C}$, se a série numérica $\sum_{n=0}^{\infty} a_{n}\left(w-z_{0}\right)^{n}$ converge (diverge), dizemos que a série de potências converge (diverge) em $w$.

Se $\sum_{n=0}^{\infty}\left|a_{n}\left(w-z_{0}\right)\right|^{n}$ converge, dizemos que a série de potências converge absolutamente em $w$.

Se $\sum_{n=0}^{\infty} a_{n}\left(z-z_{0}\right)^{n}$ converge (absolutamente) em todos os pontos de um conjunto $\Omega$, dizemos que a série converge (absolutamente) em $\Omega$.

Seja

$$
A=\left\{w \in \mathbb{C}: \sum_{n=0}^{\infty} a_{n}\left(w-z_{0}\right)^{n}<\infty\right\}
$$

Podemos definir uma função $f: A \rightarrow \mathbb{C}$ dada por $f(w)=\lim _{n \rightarrow \infty} \sum_{k=0}^{n} a_{k}\left(w-z_{0}\right)^{k}$, para todo $w \in A$. Neste caso, podemos escrever $f(z)=\sum_{n=0}^{\infty} a_{n}\left(z-z_{0}\right)^{n}$, com $z \in A$.

\section{Proposição 3.5 (Critério de Cauchy para sequência de funções).}

(a) A sequência de funções $\left(f_{n}\right)_{n \geq 0}$, definidas em $\Omega$, converge uniformemente a alguma função $f$ se e somente se para todo $\varepsilon>0$, existe $n_{0} \in \mathbb{N}$ tal que

$$
\sup \left\{\left|f_{n}(x)-f_{m}(x)\right|: x \in \Omega\right\}<\varepsilon, \text { para todos } m, n \geq n_{0}
$$

(b) A série de funçôes $\sum_{n=0}^{\infty} f_{n}$, definida em $\Omega$, é uniformemente convergente à função $f(x)=$ $\sum_{n=0}^{\infty} f_{n}(x)$ se e somente se para todo $\varepsilon>0$, existe $n_{0}$ tal que

$$
\sup \left\{\left|f_{n+1}(x)+f_{n+2}(x)+\cdots+f_{n+p}(x)\right|: x \in \Omega\right\}<\varepsilon, \text { para todos } n \geq n_{0} \text { e } p \in \mathbb{N} \text {. }
$$


O teorema a seguir será útil para determinarmos conjuntos nos quais uma dada série converge uniformemente.

Teorema 3.6 (Teste $\mathbf{M}$ de Weierstrass). Seja $\left(f_{n}\right)_{n \geq 0}$ uma sequência de funções definidas em $\Omega$. Para cada $n \in \mathbb{N}$, seja $M_{n}>0$ tal que

$$
\sup _{x \in \Omega}\left|f_{n}(x)\right| \leq M_{n}
$$

Se $\sum_{n=0}^{\infty} M_{n}$ converge, então $\sum_{n=0}^{\infty} f_{n}(x)$ é uniformemente convergente.

Não é difícil ver que $\sum_{n=0}^{\infty} a_{n}(z-a)^{n}$ converge (absolutamente) em $w \in \mathbb{C}$ se e somente se a série $\sum_{n=0}^{\infty} a_{n} z^{n}$ converge (absolutamente) em $w-a$. Assim, por diversas vezes, consideraremos apenas séries centradas em 0 .

O Teorema de Abel determina o maior subconjunto aberto do plano onde uma série converge.

Teorema 3.7 (Abel). Consideremos a série $\sum_{n=0}^{\infty} a_{n} z^{n} e$

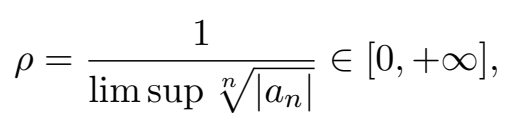

com $\rho=0$ se limsup $\sqrt[n]{\left|a_{n}\right|}=+\infty$ e $\rho=+\infty$ se limsup $\sqrt[n]{\left|a_{n}\right|}=0$. Então, vale o que segue.

(a) Se $|z|<\rho$, a série converge absolutamente.

(b) Se $|z|>\rho$, a série diverge.

(c) Se $0<r<\rho$, a série converge uniformemente em $D(0 ; r)$.

Ainda, a função $f(z)=\sum_{n=0}^{\infty} a_{n} z^{n}$ é contínua em $B(0 ; \rho)$. Ainda mais, $\rho$ é o único número que possui as propriedades $(a)$ e (b).

Demonstração. Os itens $(a)$ e $(b)$ decorrem do critério da raiz.

$\mathrm{O}$ item $(c)$ segue do item $(a)$ e do Teste M de Weierstrass.

A continuidade da função $f$ segue da proposição 3.2 (limite uniforme de funções contínuas) e a unicidade de tal $\rho$ é trivial.

Chamamos $\rho$ como acima de raio de convergência da série e $B(0 ; \rho)$ de disco de convergência da série.

O corolário a seguir decorre do critério da razão e da unicidade do raio de convergência.

Corolário 3.8. Seja $\sum_{n=0}^{\infty} a_{n} z^{n}$ uma série de potências de coeficientes não nulos. Se

$$
\lim _{n \rightarrow \infty} \frac{\left|a_{n+1}\right|}{\left|a_{n}\right|}=R \in[0,+\infty]
$$

então o raio de convergência da série é dado por $\rho=\frac{1}{R} \in[0,+\infty]$. 
As funções analíticas são localmente dadas por séries de potências. Logo, é útil termos informações sobre tais séries. Os resultados a seguir sobre séries de potências serão obtidos através da teoria de somas não ordenadas desenvolvida no capítulo 2.

Teorema 3.9 (Translação). Seja $f(z)=\sum_{n=0}^{\infty} a_{n} z^{n}$ uma série e $\rho$ seu raio de convergência. Se $z_{0} \in B(0 ; \rho)$, existe uma sequência $\left(b_{n}\right)_{n \geq 0}$ tal que, para todo $z \in B\left(z_{0} ; \rho-\left|z_{0}\right|\right)$, tem-se

$$
f(z)=\sum_{n=0}^{\infty} b_{n}\left(z-z_{0}\right)^{n}
$$

Demonstração. Seja $z \in \mathbb{C}$ com $\left|z_{0}\right|+\left|z-z_{0}\right|<\rho$.

Pelo Teorema de Abel a série é absolutamente convergente em $B(0 ; \rho)$ e portanto, pelo teorema 2.16, a série é somável. Logo,

$$
\sum_{\mathbb{N}}\left|a_{n}\right|\left(\left|z_{0}\right|+\left|z-z_{0}\right|\right)^{n}=\sum_{\mathbb{N}} \sum_{0 \leq p \leq n}\left|a_{n}\right|\left(\begin{array}{l}
n \\
p
\end{array}\right)\left|z_{0}\right|^{n-p}\left|z-z_{0}\right|^{p}<\infty
$$

Aplicando a Lei Associativa, segue que

$$
\sum_{\mathbb{N}} \sum_{0 \leq p \leq n} a_{n}\left(\begin{array}{l}
n \\
p
\end{array}\right) z_{0}^{n-p}\left(z-z_{0}\right)^{p}=\left\{\begin{array}{l}
\sum_{\mathbb{N}}\left(z_{0}+z-z_{0}\right)^{n}=\sum_{\mathbb{N}} a_{n} z^{n}=f(z) \\
\sum_{p \geq 0}\left(\sum_{n \geq p} a_{n}\left(\begin{array}{c}
n \\
p
\end{array}\right) z_{0}^{n-p}\right)^{n}\left(z-z_{0}\right)^{p}
\end{array}\right.
$$

Portanto, $f(z)=\sum_{n=0}^{\infty} b_{n}\left(z-z_{0}\right)^{n}$ em $B\left(z_{0} ; \rho-\left|z_{0}\right|\right)$, onde, $b_{n}=\sum_{k=n}^{\infty} a_{k}\left(\begin{array}{l}k \\ n\end{array}\right) z_{0}^{k-n}$, para todo $n \in \mathbb{N}$.

Teorema 3.10 (Produto de Cauchy). Sejam $f(z)=\sum_{n=0}^{\infty} a_{n} z^{n}$ e $g(z)=\sum_{n=0}^{\infty} b_{n} z^{n}$ séries convergentes em $B(0 ; r)$. Então, para todo $z \in B(0 ; r)$ temos

$$
\left(\sum_{n=0}^{\infty} a_{n} z^{n}\right)\left(\sum_{n=0}^{\infty} b_{n} z^{n}\right)=\sum_{n=0}^{\infty} c_{n} z^{n}
$$

onde $c_{n}=\sum_{l+k=n} a_{l} b_{k}$, para todo $n \in \mathbb{N}$.

Demonstração. Pelo Teorema de Abel, as séries são absolutamente convergentes em $B(0 ; r)$. Logo, aplicando o corolário 2.26 (Produto de Cauchy), dado $z \in B(0 ; r)$ temos

$$
\left(\sum_{n=0}^{\infty} a_{n} z^{n}\right)\left(\sum_{n=0}^{\infty} b_{n} z^{n}\right)=\sum_{n=0}^{\infty}\left(\sum_{l+k=n} a_{l} b_{k} z^{l+k}\right)=\sum_{n=0}^{\infty}\left(\sum_{l+k=n} a_{l} b_{k}\right) z^{n} .
$$

No teorema abaixo mostramos que compondo duas funções dadas por uma série de potências, obtemos uma nova série de potências. 
Teorema 3.11 (Composição). Sejam $f(z)=\sum_{n=0}^{\infty} a_{n} z^{n}$ e $g(z)=\sum_{n=0}^{\infty} b_{n} z^{n}$ séries convergentes em $B(0 ; r)$. Se $a_{0} \in B(0 ; r)$, existem $R>0$ e uma sequência $\left(c_{n}\right)_{n \geq 0}$ tais que

$$
g(f(z))=\sum_{n=0}^{\infty} c_{n} z^{n}, \text { para todo } z \in B(0 ; R)
$$

Demonstração. Consideremos $\rho \in \mathbb{R}$ satisfazendo $\left|a_{0}\right|<\rho<r$. Ainda, observemos que a série $\sum_{m=1}^{\infty}\left|a_{m}\right| \rho^{m}$ é convergente.

Seja $R$ tal que

$$
0<R<\frac{\rho-\left|a_{0}\right|}{1+\sum_{m=1}^{\infty}\left|a_{m}\right| \rho^{m-1}} .
$$

Então, para cada $z \in B(0 ; R)$, obtemos

$$
\sum_{m=1}^{\infty}\left|a_{m}\right||z|^{m} \leq R \sum_{m=1}^{\infty}\left|a_{m}\right| \rho^{m-1}<\rho-\left|a_{0}\right| .
$$

Portanto, $\sum_{m=0}^{\infty}\left|a_{m}\right||z|^{m}<\rho$.

Sendo $\sum_{n=0}^{\infty} b_{n} z^{n}$ somável em $B(0 ; r)$, se $|z|<R$, a seguinte soma é finita

$$
\sum_{\mathbb{N}}\left|b_{n}\right|\left(\sum_{m}\left|a_{m}\right||z|^{m}\right)^{n}=\left.\sum_{\mathbb{N}}\left|b_{n}\right| \sum_{m_{1}, m_{2}, \ldots, m_{n} \in \mathbb{N}}\left|a_{m_{1}}\right||z|^{m_{1}}\left|a_{m_{2}}\right| z\right|^{m_{2}} \cdots\left|a_{m_{n}}\right||z|^{m_{n}} .
$$

Para cada $n \in \mathbb{N}$, temos que

$$
\left|b_{n}\left(\sum_{m} a_{m} z^{m}\right)^{n}\right| \leq\left|b_{n}\right|\left(\sum_{m}\left|a_{m}\right||z|^{m}\right)^{n} .
$$

Pelo critério da comparação para somas não ordenadas, temos que

$$
\sum_{\mathbb{N}} b_{n}\left(\sum_{m} a_{m} z^{m}\right)^{n}<\infty
$$

Portanto,

$$
\begin{gathered}
g(f(z))=\sum_{n} b_{n}\left(\sum_{m} a_{m} z^{m}\right)^{n}=\sum_{n} \sum_{m_{1}, m_{2}, \ldots, m_{n}} b_{n} a_{m_{1}} z^{m_{1}} a_{m_{2}} z^{m_{2}} \cdots a_{m_{n}} z^{m_{n}}= \\
=\sum_{p}\left(\sum_{n} \sum_{m_{1}+m_{2}+\cdots+m_{n}=p} b_{n} a_{m_{1}} a_{m_{2}} \cdots a_{m_{n}}\right) z^{p} .
\end{gathered}
$$

Teorema 3.12 (Inverso algébrico). Seja $f(z)=\sum_{n=0}^{\infty} a_{n} z^{n}$ uma série convergente em $B(0 ; r)$. Se 
$a_{0} \neq 0$, existem $R>0$ e uma sequência $\left(b_{n}\right)_{n \geq 0}$ tais que

$$
\frac{1}{f(z)}=\sum_{n=0}^{\infty} b_{n} z^{n}, \text { para todo } z \in B(0 ; R) \text {. }
$$

Demonstração. Sem perda de generalidade, podemos supor $a_{0}=1$.

Consideremos as séries

$$
h(z)=1-f(z) \text { e } g(z)=\frac{1}{1-z}=\sum_{n=0}^{\infty} z^{n}
$$

definidas em alguma vizinhança da origem.

Pelo Teorema de Composição, existem $R>0$ e uma sequência $\left(b_{n}\right)_{n \geq 0}$ tais que

$$
g(1-f(z))=\frac{1}{f(z)}=\sum_{n=0}^{\infty} b_{n} z^{n}, \text { para todo } z \in B(0 ; R) .
$$

Definição 3.13. Sejam $\Omega$ aberto de $\mathbb{C}$, uma função $f: \Omega \rightarrow \mathbb{C} e z_{0} \in \Omega$. Dizemos que $f$ é derivável em $z_{0}$ se existe o limite

$$
\lim _{z \rightarrow z_{0}} \frac{f(z)-f\left(z_{0}\right)}{z-z_{0}} .
$$

Caso exista, tal limite é denotado $f^{\prime}\left(z_{0}\right)$ e é dito a derivada de $f$ em $z_{0}$.

Se $f$ for derivável em todos os pontos de $\Omega$, então $f$ é dita holomorfa (em $\Omega$ ).

Proposição 3.14. Sejam $\Omega$ aberto de $\mathbb{C}, f, g: \Omega \rightarrow \mathbb{C}$ funções, $z_{0} \in \Omega$ e $\lambda \in \mathbb{C}$. Se $f$ e $g$ são deriváveis em $z_{0}$, valem as regras abaixo.

(a) $(f+g)^{\prime}\left(z_{0}\right)=f^{\prime}\left(z_{0}\right)+g^{\prime}\left(z_{0}\right)$.

(b) $(\lambda f)^{\prime}\left(z_{0}\right)=\lambda f^{\prime}\left(z_{0}\right)$.

(c) $(f g)^{\prime}\left(z_{0}\right)=f^{\prime}\left(z_{0}\right) g\left(z_{0}\right)+f\left(z_{0}\right) g^{\prime}\left(z_{0}\right)$.

(d) Se $g\left(z_{0}\right) \neq 0$, então

$$
\left(\frac{f}{g}\right)^{\prime}\left(z_{0}\right)=\frac{f^{\prime}\left(z_{0}\right) g\left(z_{0}\right)-f\left(z_{0}\right) g^{\prime}\left(z_{0}\right)}{g\left(z_{0}\right)^{2}} .
$$

Proposição 3.15 (Regra da Cadeia). Sejam $f: \Omega_{1} \rightarrow \mathbb{C}$ e $g: \Omega_{2} \rightarrow \mathbb{C}$, com $f\left(\Omega_{1}\right) \subset \Omega_{2}$, tais que $f$ é derivável em $z_{0}$ e $g$ derivável em $f\left(z_{0}\right)$. Então, $g \circ f$ é derivável em $z_{0}$ e vale

$$
(g \circ f)^{\prime}\left(z_{0}\right)=g^{\prime}\left(f\left(z_{0}\right)\right) f^{\prime}\left(z_{0}\right) .
$$

Já vimos que uma série de potências é contínua, mostraremos a seguir que toda série de potências é também derivável no seu disco de convergência.

Teorema 3.16 (Derivação). As séries $f(z)=\sum_{n=0}^{\infty} a_{n} z^{n}$ e $g(z)=\sum_{n=1}^{\infty} n a_{n} z^{n-1}$ tem mesmo raio de convergência $\rho$ e satisfazem $f^{\prime}(z)=g(z)$, para todo $z \in B(0 ; \rho)$.

Demonstração. Pelo critério da raiz, as séries $\sum_{n=0}^{\infty} a_{n} z^{n}$ e $\sum_{n=1}^{\infty} n a_{n} z^{n}$ tem mesmo raio de convergência. 
Ainda, as séries $\sum_{n=1}^{\infty} n a_{n} z^{n}$ e $\sum_{n=1}^{\infty} n a_{n} z^{n-1}$ convergem no mesmo subconjunto de $\mathbb{C}$. Logo, $f$ e $g$ tem mesmo raio de convergência.

Dado $z_{0} \in B(0 ; \rho)$, pelo Teorema de Translação existem $r>0$ e coeficientes $\left(b_{n}\right)_{n \geq 0}$ tais que

$$
f(z)=\sum_{n=0}^{\infty} b_{n}\left(z-z_{0}\right)^{n} \text {, para todo } z \in B\left(z_{0} ; r\right) .
$$

Dado $z \in B\left(z_{0} ; r\right) \backslash\left\{z_{0}\right\}$, temos

$$
\frac{f(z)-f\left(z_{0}\right)}{z-z_{0}}=\sum_{n=1}^{\infty} b_{n}\left(z-z_{0}\right)^{n-1} .
$$

Pela continuidade das séries de potências, temos

$$
\lim _{z \rightarrow z_{0}} \frac{f(z)-f\left(z_{0}\right)}{z-z_{0}}=b_{1}
$$

Para finalizarmos, basta notarmos que pelo Teorema de Translação, temos $b_{1}=\sum_{n=1}^{\infty} n a_{n} z_{0}^{n-1}$.

Corolário 3.17 (Série de Taylor). Seja $f(z)=\sum_{n=0}^{\infty} a_{n} z^{n}$ com raio de convergência $\rho$ não nulo. Então, $f$ é infinitamente derivável em seu disco de convergência, seus coeficientes são unicamente determinados e vale

$$
f(z)=\sum_{n=0}^{\infty} \frac{f^{(n)}(0)}{n !} z^{n}, \quad \text { para todo } z \in B(0 ; \rho) .
$$

Demonstração. Pelo teorema anterior temos que $f$ é infinitamente derivável em $B(0 ; \rho)$ e, dado $k \in \mathbb{N}$, sua $k$-ésima derivada é a série

$$
f^{(k)}(z)=\sum_{n=k}^{\infty} n(n-1)(n-2) \cdots(n-(k+1)) a_{n} z^{n-k} .
$$

Logo, $a_{k}=\frac{f^{(k)}(0)}{k !}$.

No que segue, dado $\alpha \in \mathbb{R} \backslash \mathbb{N}$, usaremos a notação

$$
\left(\begin{array}{l}
\alpha \\
n
\end{array}\right)=\frac{\alpha(\alpha-1) \cdots(\alpha-(n-1))}{n !}
$$

Lema 3.18. Dado $\alpha \in \mathbb{R} \backslash \mathbb{N}$, a série de potências $B(z)=\sum_{n=0}^{\infty}\left(\begin{array}{l}\alpha \\ n\end{array}\right) z^{n}$ converge absolutamente em $B(0 ; 1)$.

Demonstração. Observemos que

$$
\left|\frac{\alpha(\alpha-1) \cdots(\alpha-n)}{(n+1) !} z^{n+1} \frac{n !}{\alpha(\alpha-1) \cdots(\alpha-(n-1)) z^{n}}\right|=\left|\frac{(\alpha-n) z}{n+1}\right| \stackrel{n \rightarrow \infty}{\rightarrow}|z| .
$$


Aplicando o critério da razão temos a convergência absoluta da série em $B(0 ; 1)$.

A proposição abaixo será utilizada na prova do Teorema de Inversão para séries de potências.

Proposição 3.19 (Expansão Binomial). Se $\alpha \in \mathbb{R} \backslash \mathbb{N}$, para todo $x \in(-1,1)$ vale a igualdade

$$
(1+x)^{\alpha}=\sum_{n=0}^{\infty}\left(\begin{array}{l}
\alpha \\
n
\end{array}\right) x^{n} .
$$

Ainda, dado $p \in \mathbb{N}, B(z)=\sum_{n=0}^{\infty}\left(\begin{array}{c}p^{-1} \\ n\end{array}\right) z^{n}$ satisfaz $B(z)^{p}=1+z$, se $|z|<1$, com $B(0)=1$.

Demonstração. Consideremos a função $f(x)=\sum_{n=0}^{\infty}\left(\begin{array}{l}\alpha \\ n\end{array}\right) x^{n}$, com $x \in(-1,1)$. Observemos que é suficiente mostrarmos que $(1+x)^{-\alpha} f(x)$ é constante e igual a 1 neste intervalo.

Pelo Teorema de Derivação, temos

$$
\begin{gathered}
(1+x) f^{\prime}(x)=\sum_{n=1}^{\infty} n\left(\begin{array}{c}
\alpha \\
n
\end{array}\right) x^{n-1}+\sum_{n=1}^{\infty} n\left(\begin{array}{l}
\alpha \\
n
\end{array}\right) x^{n}=\sum_{n=0}^{\infty}\left[(n+1)\left(\begin{array}{c}
\alpha \\
n+1
\end{array}\right)+n\left(\begin{array}{l}
\alpha \\
n
\end{array}\right)\right] x^{n}= \\
=\sum_{n=0}^{\infty}\left[(\alpha-n)\left(\begin{array}{l}
\alpha \\
n
\end{array}\right)+n\left(\begin{array}{l}
\alpha \\
n
\end{array}\right)\right] x^{n}=\alpha f(x) .
\end{gathered}
$$

Logo, temos $\left[(1+x)^{-\alpha} f(x)\right]^{\prime}=0$ com $f(0)=1$. Donde segue $f(x)=(1+x)^{\alpha}$ para todo $x \in(-1,1)$.

Provaremos a seguir a segunda afirmação.

Denotemos $b_{n}=\left(\begin{array}{c}p^{-1} \\ n\end{array}\right)$. Segue do Teorema do Produto que existem coeficientes $\left(c_{n}\right)$ tais que

$$
\left(\sum_{n=0}^{\infty} b_{n} z^{n}\right)^{p}=\sum_{n=0}^{\infty} c_{n} z^{n}, \text { se }|z|<1
$$

Por outro lado, se $x \in(-1,1)$, então temos

$$
\left(\sum_{n=0}^{\infty} b_{n} x^{n}\right)^{p}=\left[(1+x)^{\frac{1}{p}}\right]^{p}=1+x .
$$

Como $c_{n} \in \mathbb{R}$, para todo $n \in \mathbb{N}$, pela unicidade dos coeficientes (caso real), segue que $c_{0}=1$, $c_{1}=1$ e $c_{n}=0$, se $n \geq 2$.

Portanto, $B(z)^{p}=1+z$, se $|z|<1$.

No Teorema de Inversão mostraremos que uma série de potências tem uma inversa que também é uma série de potências. Tal resultado será útil para obtermos o Teorema da Função Inversa para funções analíticas. 
Teorema 3.20 (Inversão). Seja $f(z)=a_{1} z-\sum_{n=2}^{\infty} a_{n} z^{n}$, com $a_{1} \neq 0$, convergente em alguma bola aberta centrada na origem. Então, existem uma série de potências $g(z)=\sum_{n=1}^{\infty} b_{n} z^{n}$ e $r>0$, satisfazendo

$$
f(g(z))=z \text { e } g(f(z))=z \text {, para todo } z \in B(0 ; r) .
$$

Demonstração. Primeiramente vamos determinar $g$ tal que $f(g(z))=z$. Caso exista tal $g$, ela satisfaz

$$
a_{1} g(z)-a_{2} g(z)^{2}-\cdots-a_{n} g(z)^{n}-\cdots=z+0 z^{2}+0 z^{3}+\cdots .
$$

Pelo Teorema de Composição, se $\left(b_{n}\right)$ for a sequência dos coeficientes de $g$, estes devem satisfazer

$$
\left\{\begin{array}{l}
a_{1} b_{1}=1 \\
a_{1} b_{2}-a_{2} b_{1}^{2}=0, \\
a_{1} b_{3}-2 a_{2} b_{1} b_{2}-a_{3} b_{1}^{3}=0, \\
\cdot \\
\cdot \\
a_{1} b_{n}-P_{n}\left(a_{2}, \ldots, a_{n}, b_{1}, \ldots, b_{n-1}\right)=0, \\
\cdot \\
\cdot
\end{array}\right.
$$

onde $P_{n}$ é um polinômio de coeficientes em $\mathbb{N}$.

Como $a_{1} \neq 0$, os coeficientes $b_{1}, b_{2}, \ldots, b_{n}, \ldots$ estão determinados como segue

$$
b_{n}=\frac{P_{n}\left(a_{2}, \ldots, a_{n}, b_{1}, \ldots, b_{n-1}\right)}{a_{1}} .
$$

É suficiente mostrarmos que $g(z)=\sum_{n=1}^{\infty} b_{n} z^{n}$ tem raio de convergência não nulo.

Podemos assumir sem perda de generalidade que $a_{1}=1$. Nesse caso, segue que $b_{1}=1$.

Dado que limsup $\sqrt[n]{\left|a_{n}\right|}<\infty$, existe $c>0$ tal que $\left|a_{n}\right| \leq c^{n}$.

Consideremos a série, convergente em uma vizinhança da origem, dada por

$$
\psi(z)=z-\sum_{n=2}^{\infty} c^{n} z^{n}=z-\frac{c^{2} z^{2}}{1-c z}
$$

Sejam $B(z)=\sum_{n=0}^{\infty}\left(\begin{array}{c}2^{-1} \\ n\end{array}\right) z^{n}$ e $h(z)=c^{2} z^{2}-\left(4 c^{2}+2 c\right) z, \operatorname{com}|z|<1$.

Como $h(0)=0$, pelo Teorema de Composição a função $H(z)=B(h(z))$ converge em alguma bola $B(0 ; R)$. Assim, nessa bola temos $H(z)^{2}=1+h(z)$.

Temos também a convergência, em $B(0 ; R)$, da função dada por

$$
\varphi(z)=\frac{1+c z-H(z)}{2\left(c^{2}+c\right)} .
$$


Das expressões de $\psi$ e $\varphi$ segue $\psi(\varphi(z))=z$ em uma vizinhança da origem.

Sejam $d_{n}$, com $n \geq 1$, os coeficientes de $\varphi$ e denotemos $c_{n}=c^{n}$, para todo $n \geq 2$.

Logo, temos $d_{1}=1 \mathrm{e}$

$$
d_{n}=P_{n}\left(c_{2}, \ldots, c_{n}, d_{1}, \ldots, d_{n-1}\right), \text { se } n \geq 2 .
$$

Portanto, $d_{n} \geq 0$, para todo $n \in \mathbb{N}$.

Ainda, $\left|b_{1}\right|=1 \leq d_{1}=1$. Logo, considerando a expressão do polinômio $P_{n}$, obtemos indutivamente, para todo $n \in \mathbb{N}$, a desigualdade

$$
\left|b_{n}\right|=\left|P_{n}\left(a_{2}, \ldots, a_{n}, b_{1}, \ldots, b_{n-1}\right)\right| \leq P_{n}\left(c_{2}, \ldots, c_{n}, d_{1}, \ldots, d_{n-1}\right)=d_{n} .
$$

Pelo critério da comparação, a série $g(z)=\sum_{n=1}^{\infty} b_{n} z^{n}$ tem raio de convergência não nulo.

Temos $f(g(z))=z$ em alguma bola centrada na origem. Pela mesma construção, existe uma série de potências $h$ tal que $g(h(z))=z$.

Portanto, em uma vizinhança da origem, temos

$$
g(f(z))=g[f(g(h(z)))]=g[f \circ g(h(z))]=g(h(z))=z .
$$

Concluímos o desejado.

\subsection{Funções analíticas}

Alguns autores chamam de função analítica o que entendemos por função holomorfa. Estamos interessados em funções analíticas segundo Weierstrass e nesta seção estudaremos suas propriedades.

Definição 3.21. Dado um aberto $\Omega$ de $\mathbb{C}$, uma função $f: \Omega \rightarrow \mathbb{C}$ é dita analítica se puder ser escrita como uma série de potências em torno de cada ponto do seu domínio. Isto é, se para cada $z_{0} \in \Omega$, existirem $r>0$ e uma sequência $\left(a_{n}\right)_{n \geq 0}$ de números complexos tais que

$$
f(z)=\sum_{n=0}^{\infty} a_{n}\left(z-z_{0}\right)^{n}, \text { para todo } z \in B\left(z_{0} ; r\right) .
$$

Definição 3.22. Uma função $f$ é dita inteira se satizfaz $f(z)=\sum_{n=0}^{\infty} a_{n} z^{n}$, para todo $z \in \mathbb{C}$.

Pelo Teorema de Translação, temos que toda série de potências é analítica no seu disco de convergência. Segue do corolário 3.17 que se $f(z)=\sum_{n=0}^{\infty} a_{n}\left(z-z_{0}\right)^{n}$ em $B\left(z_{0} ; r\right)$, então temos $a_{k}=\frac{f^{(k)}\left(z_{0}\right)}{k !}$, para todo natural $k$.

As duas proposições a seguir decorrem das propriedades das séries para potências provadas na seção anterior. 
Proposição 3.23. Sejam $f$ e $g$ analíticas num aberto $\Omega$ e $\lambda \in \mathbb{C}$. Então, são analíticas em $\Omega$ as seguintes funções

(a) $f+g, \lambda f$ e $f g$;

(b) $\frac{1}{f}$, se $f$ não se anula.

Proposição 3.24. Sejam $g \in \mathcal{A}\left(\Omega_{1}\right)$ e $f \in \mathcal{A}\left(\Omega_{2}\right)$, com $g\left(\Omega_{1}\right) \subset \Omega_{2}$. Então, $f \circ g \in \mathcal{A}\left(\Omega_{1}\right)$.

Demonstração. Dado $z_{0} \in \Omega_{1}$, existe $r>0$ tal que $f(z)=\sum_{n=0}^{\infty} a_{n}\left(z-g\left(z_{0}\right)\right)^{n}$ em $B\left(g\left(z_{0}\right) ; r\right)$ e $g(z)=\sum_{n=0}^{\infty} b_{n}\left(z-z_{0}\right)^{n}$ em $B\left(z_{0} ; r\right)$

Também existe $\delta>0$ tal que $\left|g(z)-g\left(z_{0}\right)\right|<r$, se $\left|z-z_{0}\right|<\delta$.

Consideremos as funções definidas em $B(0 ; r)$ dadas por

$$
G(z)=g\left(z+z_{0}\right)-g\left(z_{0}\right) \text { e } F(z)=f\left(z+g\left(z_{0}\right)\right)
$$

Observemos que $G(0)=0$. Logo, pelo Teorema de Translação, existe $R \in(0, r]$ tal que

$$
F(G(z))=\sum_{n=0}^{\infty} c_{n} z^{n}, \text { para todo } z \in B(0 ; R) .
$$

Consequentemente, supondo $R<\delta$, se $\left|z-z_{0}\right|<R$ então vale a igualdade

$$
\sum_{n=0}^{\infty} c_{n}\left(z-z_{0}\right)^{n}=F\left(G\left(z-z_{0}\right)\right)=F\left(g(z)-g\left(z_{0}\right)\right)=f(g(z)) .
$$

Os dois teoremas a seguir terão grande importância no desenvolvimento deste trabalho.

Teorema 3.25 (Princípio do Prolongamento Analítico). Sejam $\Omega$ aberto conexo não vazio e $f \in \mathcal{A}(\Omega)$. As seguintes afirmações são equivalentes.

(a) $f \equiv 0$.

(b) Existe um ponto $\zeta \in \Omega$ tal que $f^{(n)}(\zeta)=0$, para todo $n \geq 0$.

(c) Existe um aberto $W \subset \Omega$ não vazio tal que $\left.f\right|_{W} \equiv 0$.

Demonstração. $(a \Rightarrow b)$ Trivial.

$(b \Rightarrow c)$ Decorre da representação em série de Taylor de uma série de potências.

$(c \Rightarrow a)$ Consideremos o conjunto

$$
A=\left\{a \in \Omega: f^{(n)}(a)=0, \text { para todo } n \in \mathbb{N}\right\}=\bigcap_{n=0}^{\infty}\left(f^{(n)}\right)^{-1}(0),
$$

que contém $W$.

Pela analiticidade de $f$ e a representação em série de Taylor, $A$ é um aberto de $\Omega$. Pela continuidade das derivadas de $f$, segue que $A$ é fechado em $\Omega$. Da conexidade de $\Omega$, segue $A=\Omega$ e $f \equiv 0$. 
A proposição abaixo garante a existência de primitivas para funções analíticas em bolas abertas.

Proposição 3.26. Valem as seguintes propriedades

(a) Se $f(z)=\sum_{n=0}^{\infty} a_{n}\left(z-z_{0}\right)^{n}$ em $B\left(z_{0} ; r\right)$, então

$$
g(z)=\sum_{n=0}^{\infty} \frac{a_{n}}{n+1}\left(z-z_{0}\right)^{n+1}
$$

é uma primitiva para $f$ na bola.

(b) Se $\Omega$ é um aberto conexo, $f \in \mathcal{A}(\Omega)$ e $f^{\prime} \equiv 0$ em $\Omega$, então $f$ é constante.

Demonstração. Dado $z \in B\left(z_{0} ; r\right)$, temos $\lim _{n \rightarrow \infty} \frac{\left|z-z_{0}\right|}{n+1}=0$. Logo, existe $M>0$ tal que $\frac{\left|z-z_{0}\right|}{n+1} \leq M$, para todo $n \in \mathbb{N}$.

Assim, temos

$$
\frac{\left|a_{n}\left(z-z_{0}\right)^{n+1}\right|}{n+1}=\frac{\left|z-z_{0}\right|}{n+1}\left|a_{n}\left(z-z_{0}\right)^{n}\right| \leq M\left|a_{n}\left(z-z_{0}\right)^{n}\right|, \text { para todo } n \in \mathbb{N} .
$$

A convergência de $\sum_{n=0}^{\infty} \frac{a_{n}}{n+1}\left(z-z_{0}\right)^{n+1}$ em $B\left(z_{0} ; r\right)$ segue do critério da comparação. A conclusão do item $(a)$ segue do Teorema de Derivação.

Para o item (b), notemos que dado $a \in \Omega$, temos $f^{(n)}(a)=0$, para todo $n \geq 1$. Logo, em uma vizinhança de $a$, pela representação em série de Taylor de $f$, temos que $f(z)=f(a)$.

Segue do Princípio do Prolongamento Analítico que $f$ é constante em $\Omega$.

Teorema 3.27 (Princípio dos Zeros Isolados). Sejam $\Omega$ aberto conexo e $f \in \mathcal{A}(\Omega)$ não nula. Então, o conjunto $Z(f)=\{w \in \Omega: f(w)=0\}$ é discreto e fechado em $\Omega$. Se a $\in Z(f)$, existem um natural $k \geq 1$ e uma função $g \in \mathcal{A}(\Omega)$ tais que

$$
f(z)=(z-a)^{k} g(z), \text { para todo } z \in \Omega, \text { com } g(a) \neq 0 .
$$

Demonstração. Sendo $f$ analítica, existe $r>0$ tal que

$$
f(z)=\sum_{n=0}^{\infty} \frac{f^{(n)}(a)}{n !}(z-a)^{n}, \text { para todo } z \in B(a ; r) .
$$

Como $f$ é não nula, pelo Princípio do Prolongamento Analítico, existe o menor natural $k \geq 1$ tal que $f^{(k)}(a) \neq 0$.

Denotando $\frac{f^{(n)}(a)}{n !}=b_{n}$, para todo $n \in \mathbb{N}$, segue que $b_{k} \neq 0$.

Ainda, dado $z \in B(a ; r)$, temos que

$$
f(z)=(z-a)^{k}\left[b_{k}+b_{k+1}(z-a)+\cdots+b_{k+n}(z-a)^{n}+\cdots\right] .
$$

Definamos a seguinte função

$$
g(z)=\left\{\begin{array}{l}
b_{k}+b_{k+1}(z-a)+\cdots+b_{k+n}(z-a)^{n}+\cdots, \text { se } z \in B(a ; r), \\
\frac{f(z)}{(z-a)^{k}}, \text { se } z \in \Omega \backslash\{a\} .
\end{array} .\right.
$$


Temos $g$ bem definida, analítica em $\Omega$, com $g(a) \neq 0$ e $f(z)=(z-a)^{k} g(z)$, para todo $z \in \Omega$.

Pela continuidade de $g$ segue que $Z(f)$ é discreto em $\Omega$. A continuidade de $f$ implica que $Z(f)$ é fechado em $\Omega$.

Corolário 3.28 (Princípio da Identidade). Sejam $\Omega$ aberto conexo e as funções $f, g \in \mathcal{A}(\Omega)$. Se $A$ é um conjunto com ponto de acumulação em $\Omega$ e $\left.f\right|_{A}=\left.g\right|_{A}$, então, $f=g$.

Demonstração. Consequência do Princípio dos Zeros Isolados.

Pelo Princípio dos Zeros Isolados segue que funções analíticas, assim como polinômios, tem zeros isolados. Logo, podemos dar uma prova para os Princípios do Módulo Máximo e Mínimo para funções analíticas, análoga a que fizemos para polinômios.

Se $f: \Omega \rightarrow \mathbb{C}$, denotamos $|f|: z \in \Omega \mapsto|f(z)|$.

Teorema 3.29 (Princípios do Módulo Máximo e Mínimo). Sejam $\Omega$ aberto conexo e $f \in$ $\mathcal{A}(\Omega)$ não constante. Nessas condições, vale o que segue.

(a) $|f|$ não tem máximo local

(b) Se $|f|$ tem um mínimo local em $z_{0}$, então, $f\left(z_{0}\right)=0$.

Demonstração 1. Sem perda de generalidade, podemos supor $z_{0}=0$ um ponto de máximo/mínimo local de $|f|$.

Portanto, em alguma bola centrada na origem, $B(0 ; \varepsilon)$, a seguinte expressão tem sinal constante

$$
|f(z)|^{2}-|f(0)|^{2}
$$

Pelo Princípio dos zeros isolados, como $f$ é não constante, existem um natural $k \geq 1$ e uma função $g \in \mathcal{A}(\Omega)$, com $g(0) \neq 0$, tais que $f(z)=f(0)+z^{k} g(z)$, para todo $z \in \Omega$.

Substituindo $z=r \omega$, com $0<r<\varepsilon$ e $\omega \in S^{1}$, obtemos

$$
|f(r \omega)|^{2}-|f(0)|^{2}=2 r^{k} \operatorname{Re}\left[\overline{f(0)} \omega^{k} g(r \omega)\right]+r^{2 k}|g(r \omega)|^{2} .
$$

Dividindo o segundo membro da expressão em 3.2 por $r^{k}$ e fixando $\omega \in S^{1}$, por continuidade temos

$$
2 \operatorname{Re}\left[\overline{f(0)} \omega^{k} g(0)\right]
$$

com o mesmo sinal de 3.1, qualquer que seja $\omega \in S^{1}$.

Consequentemente, obtemos que $\overline{f(0)} g(0)=0$. Logo, como $g(0) \neq 0$, temos $f(0)=0$.

Se $z_{0}$ é ponto de máximo local de $|f|$, pelo Princípio do Prolongamento Analítico, $f$ é nula, contradizendo nossa hipótese. Caso $z_{0}$ seja ponto de mínimo local de $|f|$, mostramos que $f\left(z_{0}\right)=0$.

Lema 3.30. Seja $F: \mathbb{C} \rightarrow[0,+\infty)$ uma função contínua tal que

$$
\lim _{|z| \rightarrow+\infty} F(z)=0
$$

Então, F assume um valor máximo global. 
Demonstração. Se $F$ é a função nula, é claro que $F$ tem valor máximo global.

Consideremos a seguir que $F$ é não nula.

Fixemos $z_{0} \in \mathbb{C}$ tal que $F\left(z_{0}\right) \neq 0$.

Devido às hipóteses, existe $R>\left|z_{0}\right|$ tal que

$$
F(z)<F\left(z_{0}\right), \text { se }|z| \geq R \text {. }
$$

Pelo Teorema do Máximo de Weierstrass aplicado à função contínua $F$ restrita ao compacto $D(0 ; R)$, existe um ponto $z_{1} \in D(0 ; R)$ tal que

$$
F(z) \leq F\left(z_{1}\right), \text { para todo } z \in D(0 ; R)
$$

Notemos que

$$
F\left(z_{0}\right) \leq F\left(z_{1}\right)
$$

Logo, se $|z| \leq R$, então $F(z) \leq F\left(z_{1}\right)$.

Caso $|z| \geq R$, temos $F(z)<F\left(z_{0}\right) \leq F\left(z_{1}\right)$.

Portanto, $z_{1}$ é ponto de máximo da função $F$ em $\mathbb{C}$.

Como consequência temos o conhecido Teorema de Liouville.

Teorema 3.31 (Liouville). Se $f(z)=\sum_{n=0}^{+\infty} a_{n} z^{n}$, para $z \in \mathbb{C}$, é uma função limitada, então $f$ é constante.

Demonstração. Por hipótese, a função $f$ é limitada. Logo, a função dada por $f(z)-a_{0}=z\left(a_{1}+\right.$ $\left.a_{2} z+a_{3} z^{2}+\cdots\right)$, onde $z \in \mathbb{C}$, é também limitada.

Seja $M>0$ tal que $\left|f(z)-a_{0}\right| \leq M$, para todo $z \in \mathbb{C}$.

Consideremos a função contínua $h(z)=\left(a_{1}+a_{2} z+a_{3} z^{2}+\cdots\right)$, com $z \in \mathbb{C}$.

Logo, se $z \neq 0$, temos

$$
|h(z)|=\frac{\left|f(z)-a_{0}\right|}{|z|} \leq \frac{M}{|z|}
$$

Portanto,

$$
\lim _{|z| \rightarrow \infty}|h(z)|=0 .
$$

Consequentemente, pelo lema anterior, segue que a função $|h|$ tem máximo global.

Pelo Princípio do Módulo Máximo, segue $h$ é constante e igual a zero e portanto, $f$ é constante.

Consequentemente, se $f$ for uma função inteira não constante então $f(\mathbb{C})$ é denso em $\mathbb{C}$. Com efeito, se existirem $c \in \mathbb{C}$ e $r>0$ tais que $B(c ; r) \cap f(\mathbb{C})=\emptyset$, então a função $g: z \in \mathbb{C} \mapsto \frac{1}{f(z)-c}$ é inteira, limitada e não constante, o que não pode ocorrer. Mais adiante provaremos um resultado mais forte que esse, o Pequeno Teorema de Picard (ver teorema A.20). 


\subsubsection{O Teorema da Aplicação Aberta e isomorfismos analíticos}

A prova do Teorema da Aplicação Aberta para funções analíticas é análoga a dada para polinômios no capítulo 1, mas preferimos discorrer novamente sobre ela.

Teorema 3.32 (Teorema da Aplicação Aberta). Sejam $\Omega$ aberto conexo e $f \in \mathcal{A}(\Omega)$ não constante. Então, $f(\Omega)$ é aberto.

Demonstração. Seja $z_{0} \in \Omega$. Pelo Princípio dos zeros isolados, existe $r>0$ tal que

$$
D\left(z_{0} ; r\right) \subset \Omega \text { e } f(z)-f\left(z_{0}\right) \neq 0 \text {, se } z \in D\left(z_{0} ; r\right) \backslash\left\{z_{0}\right\} .
$$

Consideremos o real positivo

$$
R=\min _{z \in \partial D\left(z_{0} ; r\right)} \frac{\left|f(z)-f\left(z_{0}\right)\right|}{2}
$$

e um ponto $w \in B\left(f\left(z_{0}\right) ; R\right)$.

Provaremos que $w \in f(\Omega)$. Logo, é suficiente mostrarmos que a função não constante

$$
h: z \in D\left(z_{0} ; r\right) \mapsto f(z)-w
$$

se anula.

Notemos que $\left|f\left(z_{0}\right)-w\right|<R$. Se $z \in \partial B\left(z_{0} ; r\right)$, então

$$
|f(z)-w| \geq\left|f(z)-f\left(z_{0}\right)\right|-\left|f\left(z_{0}\right)-w\right|>2 R-R=R .
$$

Assim, o ponto $w_{0}$ de mínimo da função $|h|$ na bola fechada $D\left(z_{0} ; r\right)$ está em $B\left(z_{0} ; r\right)$. Como $h$ não é constante em $B\left(z_{0} ; r\right)$, pelo Princípio do Módulo Mínimo segue $h\left(w_{0}\right)=0$. Portanto, $f\left(w_{0}\right)=w$.

Veremos uma versão do Teorema da Função Inversa para funções analíticas. A demonstração é bastante simples e decorre do Teorema de Inversão. Sem o Teorema de Inversão, apenas concluiríamos que nossa função tem uma inversa holomorfa.

A seguir faremos uso do termo holomorfa pois, optamos por enunciar a definição usualmente dada na área, porém em nosso texto, uma função holomorfa não é necessariamente analítica, uma vez que a prova de tal equivalência é feita via a Fórmula Integral de Cauchy.

Definição 3.33. Sejam $\Omega_{1}$ e $\Omega_{2}$ abertos e $f: \Omega_{1} \rightarrow \Omega_{2}$. Dizemos que $f$ é um isomorfismo analítico se $f$ é bijetora e $f$ e $f^{-1}$ são holomorfas.

Provaremos mais adiante, no Teorema da função Inversa Global (p. 40), que se $\Omega_{1}$ for conexo, $f$ analítica e bijetora, então, $f$ é um isomorfismo analítico e sua inversa é analítica.

Primeiramente estudaremos a inversão local de uma função analítica.

Teorema 3.34 (Função Inversa Local). Sejam $f \in \mathcal{A}(\Omega)$ e $a \in \Omega$ tal que $f^{\prime}(a) \neq 0$. Então, existe $r>0$ tal que

(a) $\left.f\right|_{B(a ; r)}$ é injetora e $V=f(B(a ; r))$ é aberto.

(b) $\left.f\right|_{B(a ; r)}: B(a ; r) \rightarrow V$ é um isomorfismo analítico com inversa analítica. 
Demonstração. Considerando $s>0$ tal que $B(a ; s) \subset \Omega$ e a função $g: z \in B(0 ; s) \mapsto f(z+a)-f(a)$, podemos supor, sem perda de generalidade, que $a=0$ e $f(0)=0$.

Pelo Teorema de Inversão, existem $r>0$ e $h(z)=\sum_{n=1}^{\infty} b_{n} z^{n}$ tais que $f(h(z))=z$ e $h(f(z))=z$, para todo $z \in B(0 ; r)$.

Decorre do Teorema da Aplicação Aberta (p. 39) que o conjunto $f(B(0 ; r))=V$ é aberto.

Para finalizarmos, notemos que $\left.f\right|_{B(0 ; r)}: B(0 ; r) \rightarrow V$ é bijetora e sua inversa $h$ é analítica.

Apresentaremos duas provas distintas para o teorema a seguir. A primeira pode ser vista em [19] e, utiliza que uma função analítica é localmente uma série de potências. A segunda, provada apenas no Apêndice B, é uma versão da geralmente encontrada em livros de Análise complexa, porém, diferentemente da prova usual, em nossa demonstração não faremos uso da existência de primitivas de funções holomorfas em determinados abertos apenas de séries de potências.

Teorema 3.35 (Representação Local). Sejam $f \in \mathcal{A}(\Omega)$ não constante, $z_{0} \in \Omega$ e m a ordem de $z_{0}$ como zero da função $h: z \in \Omega \mapsto f(z)-f\left(z_{0}\right)$. Então, existem um aberto $V$ contendo a origem, uma bola $B(0 ; r)$ e um isomorfismo analítico $\varphi: V \rightarrow B(0 ; r)$, com $\varphi(0)=0$ e $\varphi^{\prime}(0) \neq 0$, satisfazendo

$$
f(z)=f\left(z_{0}\right)+\left[\varphi\left(z-z_{0}\right)\right]^{m}, \text { para todo } z \in z_{0}+V .
$$

Ainda mais, dado $w \in B\left(f\left(z_{0}\right) ; r^{m}\right) \backslash\left\{f\left(z_{0}\right)\right\}$, o conjunto $f^{-1}(w) \cap\left(z_{0}+V\right)$ tem $m$ elementos.

Demonstração 1. O Princípio dos zeros isolados nos garante que podemos escrever

$$
f(z)-f\left(z_{0}\right)=a_{m}\left(z-z_{0}\right)^{m}\left[1+g\left(z-z_{0}\right)\right]
$$

para todo $z \in \Omega$, com $g \in \mathcal{A}(\Omega)$ e $g(0)=0$.

Seja $a \in \mathbb{C}$ uma raiz m-ésima de $a_{m}$. Isto é, $a^{m}=a_{m}$.

Pelo Teorema da Composição e o Teorema Binomial, dada $B(z)=\sum_{n=0}^{\infty}\left(\begin{array}{c}m^{-1} \\ n\end{array}\right) z^{n} \operatorname{com}|z|<$ 1, temos que a $B(g(z))$, em uma vizinhança da origem, é uma série de potências com raio de convergência não nulo. Nesta vizinhança temos

$$
B[g(z)]^{m}=1+g(z) .
$$

Observemos que $B(g(0))=1$. Logo, existe uma série de potências $G$ definida em alguma vizinhança $W$ da origem, com $G(0)=0$ e

$$
1+g(z)=[1+G(z)]^{m} \text {, para todo } z \in W
$$

Logo, para todo $z \in W+z_{0}$, segue

$$
f(z)=f\left(z_{0}\right)+\left[a\left(z-z_{0}\right)\left(1+G\left(z-z_{0}\right)\right)\right]^{m} .
$$

Definindo $\phi(z)=a z(1+G(z))$, com $z \in W$, temos $\phi(0)=0$ e $\phi^{\prime}(0)=a \neq 0$. Aplicando o 
Teorema da Função Inversa Local (p. 39) à $\phi$, encontramos uma bola $B(0 ; s)$ tal que

$$
\left.\phi\right|_{B(0 ; s)}: B(0 ; s) \rightarrow \phi(B(0 ; s))
$$

é um isomorfismo analítico com inversa analítica.

Como $\phi(B(0 ; s))$ é aberto, existe $r>0$ tal que

$$
B(0 ; r) \subset \phi(B(0 ; s))
$$

Definindo $V=\phi^{-1}(B(0 ; r))$ e $\varphi=\left.\phi\right|_{V}$, temos a função desejada.

Dado $w \in B\left(f\left(z_{0}\right) ; r^{m}\right) \backslash\left\{f\left(z_{0}\right)\right\}$, notemos que a equação

$$
\zeta^{m}=w-f\left(z_{0}\right)
$$

tem $m$ soluções distintas, todas na bola $B(0 ; r)$.

Com efeito, é trivial verificarmos que a equação 3.3 tem $m$ soluções distintas. Ainda, se $\omega$ é uma raiz da equação, então

$$
|\omega|^{m}=\left|w-f\left(z_{0}\right)\right|<r^{m} .
$$

Logo, como $\varphi$ é bijetora, existem exatamente $m$ pontos em $V$ tais que

$$
\varphi(z)^{m}=w-f\left(z_{0}\right) .
$$

Portanto, $f^{-1}(w) \cap\left(z_{0}+V\right)$ tem $m$ elementos.

Alguns textos provam o Teorema da Aplicação Aberta após o Teorema de Representação Local (p. 40). Mas, como provado aqui, ele pode ser obtido com ferramentas mais elementares.

Observemos que a hipótese de conexidade no teorema a seguir não será utilizada para concluir que $f^{\prime}$ não se anula, mas sim para provar que $f(\Omega)$ é aberto.

Teorema 3.36 (Função Inversa Global). Sejam $\Omega$ um aberto conexo ef $\in \mathcal{A}(\Omega)$, injetora. Então, $f^{\prime}$ não se anula, $f(\Omega)$ é aberto e $f: \Omega \rightarrow f(\Omega)$ é um isomorfismo analítico com inversa analítica.

Demonstração. Segue do Teorema da Aplicação Aberta (p. 39) que $f(\Omega)$ é aberto.

Dado $z_{0} \in \Omega$, pelo Teorema de Representação Local (p. 40) e a injetividade de $f$, temos

$$
f(z)=f\left(z_{0}\right)+\varphi\left(z-z_{0}\right)
$$

em uma vizinhança de $z_{0}$, para algum isomorfismo $\varphi$. Logo, $f^{\prime}\left(z_{0}\right)=\varphi^{\prime}(0) \neq 0$.

Dado que a analiticidade é de caráter local, pelo Teorema da Função Inversa Local (p. 39), temos que $f^{-1}$ é analítica. 


\subsubsection{A Desigualdade de Cauchy}

Para provarmos a Desigualdade de Cauchy faremos uso da Desigualdade de Gutzmer-Parseval. Geralmente, a Desigualdade de Gutzmer-Parseval só é demonstrada após a Fórmula Integral de Cauchy como consequência da Identidade de Gutzmer-Parseval. Aqui, apenas usaremos a versão da desigualdade para polinômios.

O nome Desigualdade de Gutzmer - Parseval foi sugerido pelo professor R. B. Burckel em contato eletrônico.

Teorema 3.37 (Desigualdade de Gutzmer - Parseval). Seja $f(z)=\sum_{n=0}^{\infty} a_{n}\left(z-z_{0}\right)^{n}$ convergente em $B\left(z_{0} ; \rho\right)$, com $\rho>0$. Dado $r$ tal que $0 \leq r<\rho$, definamos

$$
M(r)=\max _{\left|z-z_{0}\right|=r}|f(z)| .
$$

Então, vale

$$
\sum_{n=0}^{\infty}\left|a_{n}\right|^{2} r^{2 n} \leq M(r)^{2}
$$

Demonstração. Seja $z \in \mathbb{C}$ tal que $|z|=r$.

Dado $N \in \mathbb{N}$, pela desigualdade triangular, temos que

$$
\left|\sum_{n=0}^{N} a_{n}\left(z-z_{0}\right)^{n}\right| \leq M(r)+\left|\sum_{n=N+1}^{\infty} a_{n}\left(z-z_{0}\right)^{n}\right| \leq M(r)+\sum_{n=N+1}^{\infty}\left|a_{n}\right| r^{n}
$$

Pela desigualdade de Gutzmer-Parseval para polinômios (p. 12), para todo $N \in \mathbb{N}$, temos

$$
\sum_{n=0}^{N}\left|a_{n}\right|^{2} r^{2 n} \leq\left(M(r)+\sum_{n=N+1}^{\infty}\left|a_{n}\right| r^{n}\right)^{2}
$$

Observemos que $\lim _{N \rightarrow \infty} \sum_{n=N+1}^{\infty}\left|a_{n}\right| r^{n}=0$

Sugerimos ao leitor comparar nossa fácil prova do teorema a seguir com a apresentada em [7]. Para simplificar, usaremos com frequência a notação $\|f\|_{A}=\max \{|f(z)|: z \in A\}$.

Teorema 3.38 (Desigualdades de Cauchy para séries). Seja $f(z)=\sum_{n=0}^{\infty} a_{n}\left(z-z_{0}\right)^{n}$ convergente em $B\left(z_{0} ; \rho\right)$, com $\rho>0$. Considerando $0 \leq r<R<\rho$, temos que para todo $n \in \mathbb{N}$, valem as desigualdades

$$
\left\|f^{(n)}\right\|_{D\left(z_{0} ; r\right)} \leq \frac{n !}{(R-r)^{n}}\|f\|_{\partial D\left(z_{0} ; R\right)} \quad e \quad\left|a_{n}\right| \leq \frac{\|f\|_{\partial D\left(z_{0} ; R\right)}}{R^{n}} .
$$

Demonstração. Basta analisarmos o caso em que $f$ é não constante.

Seja $a \in D\left(z_{0} ; r\right)$. A série de Taylor de $f$ em torno de $a$ é dada por

$$
f(z)=\sum_{n=0}^{\infty} \frac{f^{(n)}(a)}{n !}(z-a)^{n}, \text { se }|z-a| \leq R-r .
$$


Por Gutzmer-Parseval (p. 41) aplicado a série em 3.4, pelo Princípio do Módulo Máximo (p. 37) e observando que se $|z-a| \leq R-r$ então $\left|z-z_{0}\right| \leq R$, temos

$$
\frac{\left|f^{(n)}(a)\right|}{n !}|z-a| \leq\|f\|_{\partial D(a ; R-r)} \leq\|f\|_{\partial D(0 ; R)}, \text { se }|z-a| \leq R-r .
$$

Computando o limite para $|z-a| \rightarrow(R-r)^{-}$, obtemos

$$
\left|f^{(n)}(a)\right| \leq \frac{n !}{(R-r)^{n}}\|f\|_{\partial D\left(z_{0} ; R\right)} .
$$

Em [28], Whyburn demonstra que o resultado a seguir é válido para funções holomorfas. Sabendo que funções analíticas são também holomorfas, pudemos adaptar sua prova.

Convém notar que no teorema 3.39, $f$ é uma função analítica numa bola e, como ainda não demonstramos que $f$ é dada por sua série de Taylor centrada na origem em todo a bola, não podemos aplicar a Desigualdade de Cauchy para séries.

Teorema 3.39 (Whyburn). Sejam $f \in \mathcal{A}(B(0 ; R)), r \in(0, R)$ e $n \in \mathbb{N}$. Então

$$
\frac{\left|f^{(n)}(0)\right|}{n !} \leq \frac{\|f\|_{\partial D(0 ; r)}}{r^{n}}
$$

Demonstração. Se $f^{(n)}(0)=0$ a desigualdade é verdadeira e pelo Princípio do Módulo Máximo (p. $37)$, se $n=0$, também.

Suponhamos que $f^{(n)}(0) \neq 0$ e $n \geq 1$.

Seja $\omega$ uma raiz primitiva das raízes $2 n$-ésimas da unidade e

$$
g(z)=\sum_{k=0}^{2 n-1}(-1)^{k} f\left(z \omega^{k}\right), \text { para todo } z \in B(0 ; R) .
$$

Temos $g \in \mathcal{A}(B(0 ; R))$. Como $\omega^{n}=-1$ e $\omega^{k} \neq-1$, para $0 \leq k<n$, então

$$
g^{(j)}(0)=\sum_{k=0}^{2 n-1}(-1)^{k} \omega^{j k} f^{(j)}(0)=\left\{\begin{array}{l}
f^{(j)}(0) \frac{1-\omega^{j 2 n}}{1+\omega^{j}}=0, \text { se } 0 \leq j<n, \\
2 n f^{(n)}(0), \text { se } j=n .
\end{array}\right.
$$

Pelo Princípio dos Zeros isolados existe $h \in \mathcal{A}(B(0 ; R)) \operatorname{com} h(0)=\frac{g^{(n)}(0)}{n !}=2 n \frac{f^{(n)}(0)}{n !}$ e tal que, para todo $z \in B(0 ; R)$, vale

$$
g(z)=z^{n} h(z)
$$

Pelo Princípio do Módulo Máximo, segue que

$$
2 n \frac{\left|f^{(n)}(0)\right|}{n !} \leq\|h\|_{\partial D(0 ; r)}=\frac{\|g\|_{\partial D(0 ; r)}}{r^{n}} \leq 2 n \frac{\|f\|_{\partial D(0 ; r)}}{r^{n}} .
$$

O Teorema de Hurwitz nos permite trabalhar com bolas maximais contidas no domínio de uma função analítica e segue do teorema anterior. 
Teorema 3.40 (Hurwitz). Sejam $\Omega$ aberto de $\mathbb{C}$, uma função $f \in \mathcal{A}(\Omega)$ e $z_{0} \in \Omega$. Então,

$$
f(z)=\sum_{n=0}^{\infty} \frac{f^{(n)}\left(z_{0}\right)}{n !}\left(z-z_{0}\right)^{n},
$$

para todo $z \in B\left(z_{0} ; r\right)$, onde $B\left(z_{0} ; r\right)$ é a maior bola aberta centrada em $z_{0}$ e contida em $\Omega$.

Demonstração. Por simplicidade, suporemos $z_{0}=0$.

Fixemos $\delta \in \mathbb{R}$ com $0<\delta<r$. Seja $M(\delta)=\|f\|_{\partial D(0 ; \delta)}$.

Aplicando o teorema 3.39, temos, para todo $n \in \mathbb{N}$,

$$
\frac{\left|f^{(n)}(0)\right|}{n !} \delta^{n} \leq M(\delta)
$$

Seja $\rho$ o raio de convergência da série $\sum_{n=0}^{\infty} \frac{f^{(n)}(0)}{n !} z^{n}$.

Caso $\rho=\infty$. Segue do Princípio do Prolongamento Analítico.

Caso $\rho<\infty$. Denotando $a_{n}=\frac{f^{(n)}(0)}{n !}$, onde $n \in \mathbb{N}$, pelo Teorema de Abel temos

$$
\frac{1}{\rho}=\limsup \sqrt[n]{\left|a_{n}\right|} \leq \limsup \frac{\sqrt[n]{M(\delta)}}{\delta}=\frac{1}{\delta}
$$

Logo, dado $0<\delta<r$, obtivemos que $\delta \leq \rho$, portanto, $\rho \geq r$.

Pelo Princípio do Prolongamento Analítico, segue que

$$
f(z)=\sum_{n=0}^{\infty} \frac{f^{(n)}(0)}{n !} z^{n} \text {, para todo } z \in B\left(z_{0} ; r\right) .
$$

Segue do Teorema de Hurwitz e do Teorema de Translação (p. 28) que uma função $f$ é analítica em $\mathbb{C}$ se e somente se é inteira. Portanto, se $f$ é analítica em $\mathbb{C}$, então

$$
f(z)=\sum_{n=0}^{\infty} \frac{f^{(n)}(0)}{n !} z^{n}, \text { para todo } z \in \mathbb{C}
$$

Corolário 3.41 (Desigualdade de Cauchy para funções analíticas). Sejam $\Omega$ aberto de $\mathbb{C}$, uma função $f \in \mathcal{A}(\Omega)$ e $z_{0} \in \Omega$. Se $D\left(z_{0} ; r\right) \subset \Omega$, então

$$
\frac{\left|f^{(n)}\left(z_{0}\right)\right|}{n !} \leq \frac{\|f\|_{\partial D\left(z_{0} ; r\right)}}{r^{n}}
$$

Corolário 3.42 (Estimativas de Cauchy sobre os compactos). Seja $f \in \mathcal{A}(\Omega), K \subset \Omega$ um compacto e $L$ uma vizinhança compacta de $K$ contida em $\Omega$. Então, dado $n \in \mathbb{N}$, existe $M(n) \geq 0$ tal que

$$
\left\|f^{(n)}\right\|_{K} \leq M(n)\|f\|_{L} .
$$

Demonstração. Fixemos $n \in \mathbb{N}$ e seja $2 r=d(K, \partial L)>0$. Então, para todo $z_{0} \in K$, temos que $D\left(z_{0} ; r\right) \subset L$. 
Pela Desigualdade de Cauchy (p. 41), para cada $z_{0} \in K$, temos a desigualdade

$$
\left|f^{(n)}\left(z_{0}\right)\right| \leq n ! \frac{\|f\|_{\partial D\left(z_{0} ; r\right)}}{r^{n}} \leq \frac{n !}{r^{n}}\|f\|_{L}
$$

\subsubsection{O Lema de Schwarz}

A versão do Lema de Schwarz que apresentamos é mais geral que a encontrada na literatura de Análise Complexa. Para concluírmos que $\left|f^{\prime}(0)\right| \leq 1$ não utilizaremos o Princípio do Módulo Máximo, mas, a Desigualdade de Gutzmer-Parseval (p. 42).

Lema 3.43 (Schwarz). Seja $f \in \mathcal{A}(B(0 ; 1))$ satisfazendo $f(0)=0$ e $|f(z)| \leq 1$, para todo $z \in B(0 ; 1)$. Nessas condições, vale o seguinte

$$
\sum_{n=0}^{\infty}\left|\frac{f^{(n)}(0)}{n !}\right|^{2} \leq 1 \quad e \quad|f(z)| \leq|z|, \text { para todo } z \in B(0 ; 1)
$$

Ainda mais, ocorre

(a) $\left|\frac{f^{(n)}(0)}{n !}\right|=1$ para algum $n \in \mathbb{N}$ se e somente se existe $\omega \in S^{1}$ tal que $f(z)=\omega z^{n}$, se $|z|<1$.

(b) $|f(z)|=|z|$, para algum $z \neq 0$ se e somente se existe $\omega \in S^{1}$ tal que $f(z)=\omega z$, se $|z|<1$.

Demonstração. Pelo Teorema de Hurwitz (p. 44), em $B(0 ; 1)$, temos $f(z)=\sum_{n=1}^{\infty} a_{n} z^{n}$, onde $a_{n}=$ $\frac{f^{(n)}(0)}{n !}$, para todo $n \geq 0$.

Segue da Desigualdade de Gutzmer-Parseval (p. 42) que

$$
\sum_{n=1}^{\infty}\left|a_{n}\right|^{2} r^{2 n} \leq 1, \text { se } 0 \leq r<1
$$

Fazendo $r \rightarrow 1^{-}$, temos que $\sum_{n=1}^{\infty}\left|a_{n}\right|^{2} \leq 1\left(\mathrm{e}\left|f^{\prime}(0)\right| \leq 1\right)$.

Se $\left|a_{n}\right|=1$, para algum $n \in \mathbb{N}$, então temos $a_{k}=0$ para todo $k \neq n$. Donde segue $f(z)=a_{n} z^{n}$, para todo $z \in B(0 ; 1)$.

Consideremos $z_{0} \in S(0 ; r)$ fixado, com $0<r<1$. Então,

$$
\left|\sum_{n=1}^{\infty} a_{n} z_{0}^{n-1}\right|=r^{-1}\left|\sum_{n=1}^{\infty} a_{n} z_{0}^{n}\right| \leq r^{-1}
$$

Pelo Princípio do Módulo Máximo (p. 37) temos $\left|\sum_{n=1}^{\infty} a_{n} z^{n-1}\right| \leq r^{-1}$, se $|z| \leq r$.

Dada a arbitrariedade de $r$, segue

$$
\left|\sum_{n=1}^{\infty} a_{n} z^{n-1}\right| \leq 1 \text {, para todo } z \in B(0 ; 1) .
$$

Logo, temos $|f(z)| \leq|z|$ para todo $|z|<1$. 
Caso $|f(z)|=|z|$ para algum $z \neq 0$, temos

$$
\left|\sum_{n=1}^{\infty} a_{n} z^{n-1}\right|=1 .
$$

Mas por 3.5 e o Princípio do Módulo Máximo, temos que existe $\omega \in S^{1}$ tal que $\sum_{n=1}^{\infty} a_{n} z^{n-1}=\omega$. Logo, $f(z)=\omega z$, se $|z|<1$.

Como consequência do Lema de Schwarz, temos a Desigualdade de Borel-Carathéodory.

Corolário 3.44 (Desigualdade de Borel-Carathéodory). Seja $f$ uma função analítica e não constante num aberto contendo $D(0 ; R)$. Se $0<r<R$, então

$$
\|f\|_{\partial D(0 ; r)} \leq \frac{2 r}{R-r} \max _{|z| \leq R} \operatorname{Ref}(z)+\frac{R+r}{R-r}|f(0)| .
$$

Demonstração. Seja $M=\max _{|z| \leq R} \operatorname{Re} f(z)$. Suponhamos que $f(0)=0$. Como $f$ é aberta, temos $M>0$.

Ainda, como $\operatorname{Re}(2 M-f(z)) \geq M>0$, se $|z| \leq R$, temos que a função

$$
g(z)=\frac{f(z)}{2 M-f(z)}
$$

é analítica em $B(0 ; R)$ e contínua em $D(0 ; R)$.

Observemos que se $|z| \leq R$, então $|2 M-f(z)| \geq|f(z)|$. Logo, $\|g\|_{D(0 ; R)} \leq 1$.

Pelo Lema de Schwarz, temos que

$$
g(z) \mid \leq \frac{|z|}{R}, \quad \text { se }|z|<R .
$$

Portanto, se $|w|=r$, segue que

$$
\frac{|f(w)|}{|2 M-f(w)|} \leq \frac{r}{R}
$$

Logo, $|f(w)| \leq \frac{r}{R}(2 M+|f(w)|)$ e

$$
\|f\|_{\partial D(0 ; r)} \leq \frac{2 r}{R-r} M
$$

Se $f(0)$ assume um valor qualquer, aplicamos o caso anterior à função $g(z)=f(z)-f(0)$.

Como $\max _{|z| \leq R} \operatorname{Re} g(z) \leq \max _{|z| \leq R} \operatorname{Re} f(z)+|f(0)|$, se $|z|=r$, tem-se que

$$
|f(z)-f(0)| \leq \frac{2 r}{R-r}(M+|f(0)|) .
$$

Por fim,

$$
\|f\|_{\partial D(0 ; r)} \leq \frac{2 r}{R-r}(M+|f(0)|)+|f(0)|=\frac{2 r}{R-r} \max _{|z| \leq R} \operatorname{Re} f(z)+\frac{R+r}{R-r}|f(0)| .
$$




\subsubsection{Convergência compacta}

Uma sequência de funções converge compactamente em $\Omega$ se converge uniformemente sobre cada compacto $K \subset \Omega$. Dizemos também que $\left(f_{n}\right)$ converge compactamente à $f$ em $\Omega$ se para cada compacto $K \subset \Omega$ a sequência $\left(\left.f_{n}\right|_{K}\right)$ converge uniformemente à $\left.f\right|_{K}$.

Proposição 3.45. Sejam $\left(f_{n}\right)$ sequência de funções analíticas num aberto conexo $\Omega$ e $u_{n}=R e f_{n}$, para todo $n \in \mathbb{N}$. Suponhamos que $u_{n}$ converge compactamente em $\Omega$ e existe $\alpha \in \Omega$ tal que $\left(f_{n}(\alpha)\right)$ converge. Nessas condições, $\left(f_{n}\right)$ converge compactamente em $\Omega$ e a função $f=\lim _{n \rightarrow \infty} f_{n}$ é contínua.

Demonstração. Dividiremos a prova em duas partes.

Seja $U$ o conjunto dos pontos $z \in \Omega$ tais que $\left(f_{n}\right)$ converge uniformemente em alguma vizinhança de $z$ contida em $\Omega$.

Afirmação 1. O conjunto $U$ é não vazio.

Sem perda de generalidade, podemos supor $\alpha=0$. A seguir mostraremos que $0 \in U$.

Dado $D(0 ; r) \subset \Omega$, consideremos $R>r$ tal que $D(0 ; R) \subset \Omega$.

Seja $\varepsilon>0$. Como $\left(u_{n}\right)$ converge uniformemente em $D(0 ; R)$ e a sequência numérica $\left(f_{n}(0)\right)$ converge, existe $n_{0} \in \mathbb{N}$ tal que

$$
\begin{gathered}
\left|u_{n}(z)-u_{m}(z)\right|<\varepsilon, \text { se } n, m \geq n_{0} \text { e } z \in D(0 ; R), \\
\left|f_{n}(0)-f_{m}(0)\right|<\varepsilon, \text { se } n, m \geq n_{0} .
\end{gathered}
$$

Pela Desigualdade de Borel-Carathéodory, o Princípio do Módulo Máximo (p. 37) e as conclusões anteriores, se $n, m \geq n_{0}$ e $z \in D(0 ; r)$, temos

$$
\begin{gathered}
\left|f_{n}(z)-f_{m}(z)\right| \leq\left\|f_{n}-f_{m}\right\|_{\partial B(0 ; r)} \leq \frac{2 r}{R-r} \max _{|z| \leq R}\left[u_{n}(z)-u_{m}(z)\right]+\frac{R+r}{R-r}\left|f_{n}(0)-f_{m}(0)\right|< \\
<\frac{2 r}{R-r} \varepsilon+\frac{R+r}{R-r} \varepsilon=\frac{3 r+R}{R-r} \varepsilon .
\end{gathered}
$$

Portanto, $\left(f_{n}\right)$ converge uniformemente em $D(0 ; r)$. Pela definição do conjunto $U$ temos $0 \in U$.

Antes de prosseguirmos lembremos que convergência uniforme implica convergência simples. Logo, se $z_{0} \in U$ então $\left(f_{n}\left(z_{0}\right)\right)$ converge. Portanto, seguindo raciocínio análogo ao que fizemos para o ponto $\alpha=0$, é possível provar que $\left(f_{n}\right)$ converge uniformemente em qualquer bola fechada centrada em $z_{0}$ e contida em $\Omega$.

Afirmação 2. $O$ conjunto $U$ é aberto e fechado em $\Omega$.

Sejam $w_{0} \in U$ e $B\left(w_{0} ; r\right) \subset \Omega$ tal que $\left(f_{n}\right)$ converge uniformemente em $B\left(w_{0} ; r\right)$. Então, $B\left(w_{0} ; r\right) \subset U$ e, portanto, $U$ é aberto.

Consideremos $w_{0} \in \Omega \backslash U$ e $r>0$ tal que $D\left(w_{0} ; 3 r\right) \subset \Omega$. É claro que $B\left(w_{0} ; r\right) \subset \Omega \backslash U$, caso contrário, existe $z_{0} \in B\left(w_{0} ; r\right) \cap U$. Como $D\left(z_{0} ; r\right) \subset \Omega$ e $z_{0} \in U$, segue que $\left(f_{n}\right)$ converge uniformemente em $D\left(z_{0} ; r\right)$. Mas, $w_{0} \in B\left(z_{0} ; r\right)$ e, portanto, $w_{0} \in U$. Isto contraria nossa hipótese. Logo, $U$ é fechado em $\Omega$. 
Portanto, como $\Omega$ é conexo e $U$ é não vazio, temos que $U=\Omega$. Consequentemente, a sequência $\left(f_{n}\right)$ converge compactamente em $\Omega$. Ainda, pela proposição 3.2 temos que a função $f$ definida por $f(z)=\lim _{n \rightarrow \infty} f_{n}(z)$ é contínua em $\Omega$.

Na proposição anterior, apesar de considerarmos uma sequência de funções analiticas, apenas concluímos que a função $f$ é contínua. Após provarmos o Teorema da Série Dupla de Weierstrass, acrescentaremos que $f$ é analítica em $\Omega$. Ainda mais, que $\left(f_{n}^{(k)}\right)$ converge compactamente à $f^{(k)}$, para todo $k \in \mathbb{N}$.

Se $F(z)=\sum_{m=0}^{\infty} f_{m}(z)$ e cada $f_{m}$ é analítica, é razoável esperar que $F$ seja analítica e satisfaça

$$
F^{(k)}(z)=\sum_{m=0}^{\infty} f_{m}^{(k)}(z)
$$

No teorema abaixo analisamos esta situação.

Teorema 3.46 (Série Dupla de Weierstrass). Seja $\sum_{m=0}^{\infty} f_{m}(z)$ uma série de funções, onde cada função é dada por uma série $f_{m}(z)=\sum_{n=0}^{\infty} a_{n}(m) z^{n}$ convergente em $B(0 ; R)$. Suponhamos que $\sum_{m=0}^{\infty} f_{m}(z)$ converge uniformemente em cada $D(0 ; \rho)$, com $0<\rho<R$. Seja $F(z)=\sum_{m=0}^{\infty} f_{m}(z)$ a função limite definida em $B(0 ; R)$. Então, $F$ é analítica em $B(0 ; R)$ e se $|z|<R$, temos as igualdades

$$
F(z)=\sum_{n=0}^{\infty}\left[\sum_{m=0}^{\infty} a_{n}(m)\right] z^{n} \quad \text { e } F^{(k)}(z)=\sum_{m=0}^{\infty} f_{m}^{(k)}(z), k \in \mathbb{N} .
$$

Ainda mais, para cada $k \in \mathbb{N}$, a série $\sum_{m=0}^{\infty} f_{m}^{(k)}(z)$ converge uniformemente à função $F^{(k)}$ em $D(0 ; \rho)$, onde $0<\rho<R$.

Demonstração. Seja $0<\rho<r<R$. Por hipótese, a sequência $s_{m}=\sum_{k=0}^{m} f_{k}$ converge uniformemente à $F$ em $D(0 ; r)$. Pelo critério de Cauchy, dado $\varepsilon>0$, existe $m_{0} \in \mathbb{N}$, tal que se $m \geq m_{0}, p \in \mathbb{N}$ e $|z| \leq r$, então

$$
\left|f_{m+1}(z)+f_{m+2}(z)+\cdots+f_{m+p}(z)\right|<\varepsilon
$$

Portanto, $\left|\sum_{n=0}^{\infty}\left(a_{n}(m+1)+\cdots+a_{n}(m+p)\right) z^{n}\right| \leq \varepsilon$, se $|z| \leq r$.

Pela Desigualdade de Gutzmer-Parseval (p. 42), para $m \geq m_{0}, p \in \mathbb{N}$ e $|z| \leq r$, obtemos

$$
\sum_{n=0}^{\infty}\left|a_{n}(m+1)+\cdots+a_{n}(m+p)\right|^{2}|z|^{2 n} \leq \varepsilon^{2} .
$$

Assim, se $|z| \leq \rho, n, p \in \mathbb{N}$ e $m \geq m_{0}$, então

$$
\left|a_{n}(m+1)+\cdots+a_{n}(m+p)\right||z|^{n}=\sqrt{\left|a_{n}(m+1)+\cdots+a_{n}(m+p)\right|^{2} r^{2 n}}\left(\frac{|z|}{r}\right)^{n} \leq \varepsilon\left(\frac{\rho}{r}\right)^{n} .
$$


Logo, pelo Critério de Cauchy para séries numéricas

$$
\sum_{m=0}^{\infty} a_{n}(m)<\infty, \text { para todo } n \in \mathbb{N} \text {. }
$$

Mostraremos que a função $F$ é analítica em $B(0 ; R)$ e sua série tem coeficientes dados por $\sum_{m=0}^{\infty} a_{n}(m)$, para todo $n \in \mathbb{N}$.

Pelo que fizemos, se $m \geq m_{0}, z \in D(0 ; \rho)$ e $n \in \mathbb{N}$

$$
\left|\sum_{\mu=m+1}^{\infty} a_{n}(\mu)\right||z|^{n} \leq \varepsilon\left(\frac{\rho}{r}\right)^{n}
$$

Fixando $z \in D(0 ; \rho)$ e $m \geq m_{0}$, segue da desigualdade anterior que

$$
\sum_{n=0}^{\infty}\left|\sum_{\mu=0}^{\infty} a_{n}(\mu)-\sum_{\mu=0}^{m} a_{n}(\mu)\right||z|^{n} \leq \varepsilon\left(1-\frac{\rho}{r}\right)^{-1} .
$$

Logo, se $m \geq m_{0}$ e $z \in D(0 ; \rho)$, segue

$$
\begin{gathered}
\left|\sum_{n=0}^{\infty}\left[\sum_{\mu=0}^{\infty} a_{n}(\mu)-\sum_{\mu=0}^{m} a_{n}(\mu)\right] z^{n}\right| \leq \varepsilon\left(1-\frac{\rho}{r}\right)^{-1}, \\
\left|\sum_{n=0}^{\infty}\left[\sum_{\mu=0}^{\infty} a_{n}(\mu)\right] z^{n}-\sum_{n=0}^{\infty} \sum_{\mu=0}^{m} a_{n}(\mu) z^{n}\right| \leq \varepsilon\left(1-\frac{\rho}{r}\right)^{-1} .
\end{gathered}
$$

Pela definição das funções $f_{\mu}$, se $m \geq m_{0}$ e $z \in D(0 ; \rho)$ temos

$$
\left|\sum_{n=0}^{\infty}\left[\sum_{\mu=0}^{\infty} a_{n}(\mu)\right] z^{n}-\sum_{\mu=0}^{m} f_{\mu}(z)\right| \leq \varepsilon\left(1-\frac{\rho}{r}\right)^{-1} .
$$

Portanto, computando o limite para $m \rightarrow+\infty$ na desigualdade 3.6, temos

$$
\left|\sum_{n=0}^{\infty}\left[\sum_{\mu=0}^{\infty} a_{n}(\mu)\right] z^{n}-F(z)\right|<\varepsilon\left(1-\frac{\rho}{r}\right)^{-1}, \text { para todo } z \in B(0 ; R) .
$$

Logo, $F$ é analítica em $B(0 ; R)$.

Para finalizarmos, consideremos $r, \rho \in \mathbb{R}$ tais que $0<\rho<r<R$. Como $\left(s_{m}-F\right)$ converge uniformemente à função nula em $D(0 ; r)$, fixado $k \in \mathbb{N}$, segue da desigualdade de Cauchy (p. 41) que

$$
\left\|s_{m}^{(k)}-F^{(k)}\right\|_{D(0 ; \rho)} \leq \frac{k !}{(r-\rho)^{k}}\left\|s_{m}(z)-F(z)\right\|_{\partial D(0 ; r)} .
$$

Portanto, a sequência $\left(s_{m}^{(k)}\right)_{m}$ converge uniformemente à $F^{(k)}$ em $D(0 ; \rho)$, qualquer que seja $k \in \mathbb{N}$. 
Consequentemente,

$$
F^{(k)}(z)=\sum_{m=0}^{\infty} f_{m}^{(k)}(z), \text { para todo } z \in B(0 ; R) .
$$

Observemos que provamos acima que podemos alterar a ordem do somatório. Isto é,

$$
F(z)=\sum_{m=0}^{\infty} \sum_{n=0}^{\infty} a_{n}(m) z^{n}=\sum_{n=0}^{\infty} \sum_{m=0}^{\infty} a_{n}(m) z^{n} .
$$

Aplicando o Teorema da Série Dupla de Weierstrass obtemos uma demonstração do corolário abaixo sem utilizar integração complexa.

Corolário 3.47. Sejam $\Omega$ aberto limitado em $\mathbb{C}$ e $\left(f_{n}\right)$ uma sequência de funções em $\mathcal{A}(\Omega) \cap \mathcal{C}(\bar{\Omega})$ tal que $\left(\left.f_{n}\right|_{\partial \Omega}\right)$ converge uniformemente em $\partial \Omega$. Então,

(a) $\left(f_{n}\right)$ converge compactamente em $\bar{\Omega}$.

(b) Se $f=\lim _{n \rightarrow \infty} f_{n}$, então $f \in \mathcal{A}(\Omega)$.

(c) $\left(f_{n}^{(k)}\right)$ converge compactamente em $\Omega \grave{a} f^{(k)}$, para todo $k \in \mathbb{N}$.

Demonstração. Pelo Princípio do Módulo Máximo (p. 37), temos que

$$
\left\|f_{m}-f_{n}\right\|_{\bar{\Omega}} \leq\left\|f_{m}-f_{n}\right\|_{\partial \Omega} \text {, para todos } m, n \in \mathbb{N} \text {. }
$$

Logo, $\left(f_{n}\right)$ converge compactamente em $\bar{\Omega}$.

Seja $f$ a função dada por $f(z)=\lim _{n \rightarrow \infty} f_{n}(z)$, para todo $z \in \Omega$.

Por hipótese, para cada $n \in \mathbb{N}, f_{n}$ é dada por uma série de potências em torno de $a$.

Ainda mais, dado $D(a ; r) \subset \Omega$, a série $\sum_{n=1}^{\infty}\left(f_{n+1}-f_{n}\right)+f_{1}$ tem como sequência das somas parciais

$$
s_{n}=f_{1}+\left(f_{2}-f_{1}\right)+\cdots+\left(f_{n+1}-f_{n}\right)=f_{n+1} .
$$

Observemos que $\left(s_{n}\right)$ converge uniformemente à $f$ em $D(a ; r)$.

Pelo Teorema da Série Dupla de Weierstrass temos que $f \in \mathcal{A}(B(a ; r))$ e $\left(f_{n}^{(k)}\right)$ converge compactamente à $f^{(k)}$ em $B(a ; r)$. Dado que $B(a ; r)$ é qualquer, segue que $f \in \mathcal{A}(\Omega)$ e $\left(f_{n}^{(k)}\right)$ converge compactamente à $f^{(k)}$ em $\Omega$.

O corolário a seguir será uma das ferramentas utilizadas para obtermos o Teorema da Fatoração de Weierstrass, mais precisamente, será utilizado na prova do corolário A.7. Sua prova é simples e decorre do corolário 3.47 .

Corolário 3.48. Sejam $\Omega$ aberto em $\mathbb{C}$ (não necessariamente limitado) e $\left(f_{n}\right)$ uma sequência de funções em $\mathcal{A}(\Omega)$ convergindo compactamente em $\Omega$ para $f$. Então, $f \in \mathcal{A}(\Omega)$ e $\left(f_{n}^{(k)}\right)$ converge compactamente à $f^{(k)}$ em $\Omega$. 


\subsubsection{O Teorema de Montel}

O Teorema de Montel será essencial para a demonstração do Teorema da Aplicação de Riemann.

Antes de provarmos o Teorema de Montel, façamos algumas observações. Vimos no corolário 3.48 que se uma sequência de funções analíticas converge compactamente à uma função $f$, então $f$ é analítica. Dada uma sequência de funções em $\mathcal{A}(\Omega)$, vamos analisar quais condições são suficientes para concluirmos que tal sequência converge compactamente ou que ao menos tenha uma subsequência que converge compactamente.

Observemos que se $\mathcal{F}$ é uma família em $\mathcal{A}(\Omega)$ com a propriedade de que toda sequência de elementos de $\mathcal{F}$ tem uma subsequência que converge compactamente em $\Omega$, então, fixado um compacto $K \subset \Omega$, temos

$$
\sup \left\{\|f\|_{K}: f \in \mathcal{F}\right\}<\infty .
$$

Caso contrário, existe uma sequência $\left(f_{n}\right)$ de elementos de $\mathcal{F}$ tal que $\lim _{n \rightarrow \infty}\left\|f_{n}\right\|_{K}=\infty$. Uma contradição, pois esta sequência não tem subsequência convergindo compactamente.

Consequentemente, para cada $a \in \Omega$, existem $r>0$ e uma constante $M>0$ tais que $B(a ; r) \subset \Omega$ e

$$
|f(z)| \leq M, \text { para todos } z \in B(a ; r) \text { e } f \in \mathcal{F} .
$$

Obtivemos assim uma condição necessária para que uma família $\mathcal{F}$ de funções analíticas em $\Omega$ tenha a propriedade acima mencionada. No Teorema de Montel mostraremos que esta condição, indicada em 3.7, é também suficiente.

A definição a seguir tornará o enunciado do Teorema de Montel mais simples.

Definição 3.49. Sejam $\Omega$ aberto e $\mathcal{F}$ uma família em $\mathcal{C}(\Omega)$.

(i) A família $\mathcal{F}$ é normal se toda sequência de elementos de $\mathcal{F}$ tem uma subsequência que converge compactamente em $\Omega$.

(ii) A família $\mathcal{F}$ é dita equicontínua sobre $A \subset \Omega$ se para todo $\varepsilon>0$ existe $\delta>0$ tal que para toda $f \in \mathcal{F}$ vale

$$
|f(z)-f(w)|<\varepsilon, \text { se }|z-w|<\delta \quad e \quad z, w \in A .
$$

(iii) A família $\mathcal{F}$ é localmente limitada se para todo $a \in \Omega$ existem $r>0$ e $M>0$ tais que para toda $f \in \mathcal{F}$ vale

$$
|f(z)| \leq M, \text { para todo } z \in B(a ; r) .
$$

Observemos que uma família $\mathcal{F}$ em $\mathcal{C}(\Omega)$ localmente limitada é uniformemente limitada sobre os compactos de $\Omega$. Isto é, dado $K \subset \Omega$ compacto, existe $M>0$ tal que $|f(z)| \leq M$, para todo $z \in K$ e $f \in \mathcal{F}$. Ainda mais, se $\mathcal{F}$ é uma família normal em $\mathcal{A}(\Omega)$, então $\mathcal{F}$ é localmente limitada.

Faremos uso do seguinte resultado.

Lema 3.50. Seja $\mathcal{C}=\{B(a ; r): r \in \mathbb{Q}, r>0, a \in \mathbb{Q} \times \mathbb{Q}\}$. Então, todo aberto $\Omega$ de $\mathbb{R}^{2}$ é uma reunião de bolas da coleção $\mathcal{C}$. Ainda, podemos supor o fecho das bolas em $\Omega$.

O Teorema de Montel foi publicado por Montel em 1907 e a expressão familia normal foi introduzida por ele em 1912.

Teorema 3.51 (Montel). Sejam $\Omega$ um aberto de $\mathbb{C}$ e $\mathcal{F}$ uma familia localmente limitada em $\mathcal{A}(\Omega)$. Então, 
(a) a família $\mathcal{F}$ é equicontínua sobre os compactos de $\Omega$.

(b) a família $\mathcal{F}$ é normal.

Demonstração. (a) Sejam $K \subset \Omega$ compacto e $r>0$ tal que $K(r)=\{z \in \mathbb{C}: d(z, K) \leq r\} \subset \Omega$ (é suficiente considerar $2 r=d\left(K, \Omega^{c}\right)$, se $\Omega \neq \mathbb{C}$ e $r>0$ qualquer, caso contrário).

Logo, $K(r)$ é compacto, pois é fechado e limitado. Portanto, existe $M>0$ tal que $|f(z)| \leq M$, para toda $f \in \mathcal{F}$ e todo $z \in K(r)$.

Dados $a \in K$ e $f \in \mathcal{F}$ arbitrários, consideremos a função

$$
f_{a}(z)=\frac{f(a+r z)-f(a)}{2 M}, \text { onde }|z| \leq 1 .
$$

Notando que $f_{a}(D(0 ; 1)) \subset D(0 ; 1)$ [se $z \in D(0 ; 1)$, então $\left.a+r z \in K(r)\right]$ e $f_{a}(0)=0$, segue pelo Lema de Schwarz (p. 45) que $\left|f_{a}(z)\right| \leq|z|$, para todo $z \in B(0 ; 1)$.

Seja $\varepsilon>0$. Sem perda de generalidade, suponhamos $0<\varepsilon<2 M$ e consideremos $\delta=\frac{\varepsilon r}{2 M}$.

Logo, se $z, w \in K \operatorname{com}|z-w|<\delta$, podemos escrever $z=w+r z_{0}$, com $z_{0} \in B(0 ; 1)$. Assim,

$$
\left|f_{w}\left(z_{0}\right)\right|=\frac{|f(z)-f(w)|}{2 M} \leq\left|z_{0}\right|=\frac{|z-w|}{r}<\frac{\varepsilon}{2 M} \text {, para toda } f \in \mathcal{F} .
$$

Portanto, $\mathcal{F}$ é uma família equicontínua.

(b) Dividiremos a prova em duas partes. Sejam $\left(f_{m}\right)$ sequência arbitrária em $\mathcal{F}$ e $a \in \Omega$.

Afirmação 1. Existe $\left(f_{m m}\right)$ subsequência de $\left(f_{m}\right)$ convergindo uniformemente em alguma bola fechada centrada em a.

Fixemos $r>0$ e $M>0$ tais que $D(a ; r) \subset \Omega$ e $\left|f_{m}(z)\right| \leq M$, para todos $m \in \mathbb{N}$ e $z \in D(a ; r)$. Vamos extrair de $\left(f_{m}\right)$ uma subsequência que converge uniformemente em $D\left(a ; \frac{r}{2}\right)$.

Pela Desigualdade de Cauchy (p. 41),

$$
\frac{\left|f_{m}^{(n)}(a)\right|}{n !} \leq \frac{M}{r^{n}}, \text { para todos } n, m \in \mathbb{N} .
$$

Definamos

$$
C_{n}\left[f_{m}\right]=\frac{f_{m}^{(n)}(a)}{n !}, \operatorname{com} n, m \in \mathbb{N} .
$$

Aplicaremos a seguir o Método da Diagonalização de Cantor.

A sequência numérica $\left(C_{0}\left[f_{m}\right]\right)_{m}$ é limitada. Logo, existem uma subsequência $\left(f_{0, \mu}\right)_{\mu}$ de $\left(f_{m}\right)$ e $C_{0} \in \mathbb{C}$ tais que

$$
\left(C_{0}\left[f_{0, \mu}\right]\right)_{\mu} \rightarrow C_{0}
$$

Também existe subsequência $\left(f_{1, \mu}\right)_{\mu}$ de $\left(f_{0, \mu}\right)_{\mu}$ com $\left(C_{1}\left[f_{1, \mu}\right]\right)_{\mu}$ convergindo a $C_{1} \in \mathbb{C}$, se $\mu \rightarrow$ $+\infty$. Indutivamente, para cada $m \in \mathbb{N}$, existe uma subsequência $\left(f_{m+1, \mu}\right)_{\mu}$ de $\left(f_{m, \mu}\right)_{\mu}$ com

$$
\left(C_{m+1}\left[f_{m+1, \mu}\right]\right)_{\mu}
$$

convergindo a $C_{m+1} \in \mathbb{C}$, se $\mu \rightarrow+\infty$.

Consideremos $\left(g_{n}\right)=\left(f_{n n}\right)$ subsequência de $\left(f_{m}\right)$. 
Se $N \in \mathbb{N}$ e $z \in D\left(a ; \frac{r}{2}\right)$, utilizando a expansão em série de Taylor das funções $f_{n n}$ em torno de $a$ e a desigualdade em 3.8 , dados $k, l \in \mathbb{N}$ temos

$$
\begin{gathered}
\left|g_{k}(z)-g_{l}(z)\right|=\left|\sum_{m=0}^{\infty} \frac{f_{k k}^{(m)}(a)}{m !}(z-a)^{m}-\sum_{m=0}^{\infty} \frac{f_{l l}^{(m)}(a)}{m !}(z-a)^{m}\right| \leq \\
\leq \sum_{m=0}^{N}\left|\left[\frac{f_{k k}^{(m)}(a)}{m !}-\frac{f_{l l}^{(m)}(a)}{m !}\right]\right||z-a|^{m}+\sum_{m=N+1}^{\infty} \frac{2 M}{r^{m}}|z-a|^{m} .
\end{gathered}
$$

Seja $\varepsilon>0$. Para $m_{0}$ suficientemente grande, se $N \geq m_{0}$ e $z \in D\left(a ; \frac{r}{2}\right)$, temos

$$
\sum_{m>N} \frac{2 M}{r^{m}}|z-a|^{m} \leq \sum_{m>N} 2 M \frac{1}{2^{m}}=\frac{M}{2^{N-1}}<\frac{\varepsilon}{2} .
$$

Fixemos $N=m_{0}$. Para cada $m \in\{0,1, \ldots, N\}$, como $\left(C_{m}\left[f_{k k}\right]\right)_{k}$ converge à $C_{m},\left[\left(f_{k k}\right)_{k \geq m}\right.$ é subsequência de $\left.\left(f_{m, k}\right)_{k}\right]$, existe $k(m) \in \mathbb{N}$ tal que se $k, l \geq k(m)$

$$
\left|\left[\frac{f_{k k}^{(m)}(a)}{m !}-\frac{f_{l l}^{(m)}(a)}{m !}\right]\right|\left(\frac{r}{2}\right)^{m}<\frac{\varepsilon}{2(N+1)} .
$$

Definamos $k_{0}=\max \{k(m): m=0,1, \ldots, N\}$.

Logo, se $k, l \geq k_{0}$ tem-se que

$$
\sum_{m \leq N}\left|\left[\frac{f_{k k}^{(m)}(a)}{m !}-\frac{f_{l l}^{(m)}(a)}{m !}\right]\right|\left(\frac{r}{2}\right)^{m}<\frac{\varepsilon}{2} .
$$

Assim, se $k, l \geq \max \left\{k_{0}, m_{0}\right\}$ e $z \in D\left(a ; \frac{r}{2}\right)$, então

$$
\left|g_{k}(z)-g_{l}(z)\right|<\varepsilon
$$

Portanto, $\left(g_{n}\right)$ converge uniformemente em $D\left(a ; \frac{r}{2}\right)$. Donde segue que $\left(f_{m}\right)$ tem subsequência convergindo uniformemente em $D\left(a ; \frac{r}{2}\right)$.

Afirmação 2. Existe uma subsequência $\left(h_{m m}\right)$ de $\left(f_{m}\right)$ convergindo compactamente em $\Omega$.

Pelo lema 3.50, podemos escrever

$$
\Omega=\bigcup_{n \in \mathbb{N}} B\left(a_{n} ; 2 r_{n}\right)=\bigcup_{n \in \mathbb{N}} D\left(a_{n} ; 2 r_{n}\right), \text { onde } a_{n} \in \mathbb{Q} \times \mathbb{Q}, r_{n} \in \mathbb{Q} \text {, e } r_{n}>0 \text {. }
$$

Para finalizarmos, é suficiente determinarnos uma subsequência de $\left(f_{n}\right)$ que convirja uniformemente sobre cada uma das bolas $B\left(a_{m} ; r_{m}\right)$, com $m \in \mathbb{N}$.

Pelo que fizemos anteriormente, existe subsequência $\left(h_{0, m}\right)$ de $\left(f_{m}\right)$ que converge uniformemente em $D\left(a_{0} ; r_{0}\right)$. Indutivamente, para cada $n \in \mathbb{N}$, existe subsequência $\left(h_{n+1, m}\right)_{m}$ de $\left(h_{n, m}\right)_{m}$ que converge uniformemente em $D\left(a_{n+1} ; r_{n+1}\right)$.

Segue disso que $\left(h_{m m}\right)$ subsequência de $\left(f_{m}\right)$ converge uniformemente sobre cada bola fechada 
$D\left(a_{n} ; r_{n}\right)$, pois para cada $n \in \mathbb{N},\left(h_{m m}\right)_{m \geq n}$ é subsequência de $\left(h_{n m}\right)_{m}$

$$
\begin{aligned}
& \begin{array}{llllllll}
\mathbf{h}_{\mathbf{0 0}} & h_{01} & h_{02} & h_{03} & \ldots & h_{0 m} & \ldots
\end{array} \\
& \begin{array}{llllllll}
h_{10} & \mathbf{h}_{11} & h_{12} & h_{13} & \ldots & h_{1 m} & \ldots
\end{array} \\
& \begin{array}{llllllll}
h_{20} & h_{21} & \mathbf{h}_{\mathbf{2 2}} & h_{23} & \ldots & h_{2 m} & \ldots
\end{array} \\
& \begin{array}{lllllllll}
h_{m 0} & h_{m 1} & h_{m 2} & h_{m 3} & \ldots & \mathbf{h}_{\mathbf{m m}} & \ldots
\end{array}
\end{aligned}
$$

Por fim, se $K \subset \Omega$ é um compacto arbitrário, existe $k \in \mathbb{N}$ tal que

$$
K \subset B\left(a_{1} ; r_{1}\right) \cup \ldots \cup B\left(a_{k} ; r_{k}\right) \subset D\left(a_{1} ; r_{1}\right) \cup \ldots \cup D\left(a_{k} ; r_{k}\right) .
$$

Portanto, a subsequência $\left(h_{m m}\right)$ de $\left(f_{m}\right)$ converge uniformemente sobre o compacto $K \subset \Omega$. Dada a arbitrariedade de $K$, temos que a família $\mathcal{F}$ é normal.

Pelo Teorema de Arzelà-Ascoli temos que se uma família em $\mathcal{C}(\Omega)$ for localmente limitada (em $\Omega$ ) e equicontínua sobre os compactos de $\Omega$, então ela é normal. Vimos no Teorema de Montel que se a família é de funções analíticas, é suficiente exigir que ela seja localmente limitada.

No exemplo a seguir, veremos que se $\mathcal{F}$ é uma família em $\mathcal{C}(\Omega)$ localmente limitada, isto não é suficiente para que ela seja normal.

Exemplo 3.52. Consideremos a família de funções contínuas dadas por

$$
f_{n}(z)=\left\{\begin{array}{l}
z^{n}, \text { se }|z|<1 \\
1, \text { se } 1 \leq|z|<2
\end{array}\right.
$$

$\operatorname{com} n \in \mathbb{N}^{*}$

Observemos que $\left(f_{n}\right)$ converge simplesmente à função $f$ dada por

$$
f(z)=\left\{\begin{array}{l}
0, \text { se }|z|<1 \\
1, \text { se } 1 \leq|z|<2 .
\end{array}\right.
$$

Sendo $f$ não contínua e, lembrando que convergência uniforme implica convergência simples, pela proposição 3.2 nenhuma subsequência de $\left(f_{n}\right)$ converge compactamente em $B(0 ; 2)$. 


\section{Capítulo 4}

\section{O índice de um caminho fechado e aplicações}

O principal objetivo deste capítulo é demonstrar o Princípio do Argumento e o Teorema de Rouché, sem utilizar a teoria de integração. Para isto, definiremos o índice de um caminho fechado, apenas assumindo sua continuidade. Antes, faremos um breve estudo da exponencial complexa e algumas de suas aplicações.

\subsection{A exponencial e o logaritmo complexo}

Segue do Teste da Razão que o raio de convergência da série $\sum_{n=0}^{\infty} \frac{z^{n}}{n !}$ é $\rho=+\infty$. Logo, está bem definida e é inteira, a seguinte função

$$
\exp : z \in \mathbb{C} \mapsto \sum_{n=0}^{\infty} \frac{z^{n}}{n !}
$$

A função acima é dita a exponencial complexa. A seguir enunciaremos diversas propriedades dessa função, porém omitiremos as demonstrações por serem facilmente encontradas.

Proposição 4.1. A função exponencial satisfaz as seguintes propriedades.

(a) $\exp (z+w)=\exp (z) \exp (w)$, para todos $z, w \in \mathbb{C}$.

(b) $\exp (0)=1$, $\exp$ não se anula e $\exp (z)^{-1}=\exp (-z)$, para todo $z \in \mathbb{C}$.

(c) $\exp (1)=e$, onde $e=\lim _{n \rightarrow+\infty}\left(1+\frac{1}{n}\right)^{n}$.

$(d) \exp (r)=e^{r}$, para todo $r \in \mathbb{Q}$.

(e) $\overline{\exp (z)}=\exp (\bar{z}) e|\exp (z)|=\exp (\operatorname{Re}(z))$, para todo $z \in \mathbb{C}$.

$(f) \exp ^{\prime}(z)=\exp (z)$, para todo $z \in \mathbb{C}$.

Com base nos itens $(d)$ e $(f)$ (exp é contínua) da proposição 4.1, usaremos a seguinte notação

$$
\exp (z)=e^{z} \text {, para todo } z \in \mathbb{C} \text {. }
$$

Definição 4.2. As funções cosseno e seno complexas são dadas, respectivamente, por

$$
\cos (z)=\sum_{n=0}^{\infty}(-1)^{n} \frac{z^{2 n}}{(2 n) !}, \text { onde } z \in \mathbb{C}
$$




$$
\sin (z)=\sum_{n=0}^{\infty}(-1)^{2 n+1} \frac{z^{2 n+1}}{(2 n+1) !}, \text { onde } z \in \mathbb{C} .
$$

Segue do Teorema de Abel que as funções cos e sin são inteiras. Ainda, vale para todo $z \in \mathbb{C}$

$$
\begin{gathered}
e^{i z}=\cos (z)+i \sin (z) \\
\cos (z)=\frac{e^{i z}+e^{-i z}}{2} \text { e } \sin (z)=\frac{e^{i z}-e^{-i z}}{2 i} .
\end{gathered}
$$

Definimos as funções cosseno e seno reais como a restrição das funções cos e sin complexas. Dado $x \in \mathbb{R}$, temos $\cos ^{\prime}(x)=-\sin (x)$ e $\sin ^{\prime}(x)=\cos (x)$.

Proposição 4.3. Existe o menor número real estritamente positivo c tal que $\cos (c)=0$.

Definimos o número $\pi$, por $\pi=2 c$.

Proposição 4.4. Valem as seguintes propriedades.

(a) $\left|e^{i \theta}\right|=1=\cos ^{2}(\theta)+\sin ^{2}(\theta)$, para todo $\theta \in \mathbb{R}$.

(b) $e^{i \frac{\pi}{2}}=i e e^{i \pi}=-1$.

(c) A função exp é periódica com período $2 \pi i$.

(d) $\operatorname{Se} \theta \in(0,2 \pi)$, então $e^{i \theta} \neq 1$.

(e) $e^{z}=1$ se e somente se $z \in 2 \pi i \mathbb{Z}$.

(f) A função $\xi: \theta \in[0,2 \pi) \mapsto e^{i \theta} \in S^{1}$ é bijetora. Ainda mais, a imagem de $\xi$ percorre $S^{1}$ no sentido anti-horário.

(g) A função exp $: \mathbb{R} \rightarrow(0,+\infty)$ é um difeomorfismo.

(h) A imagem da função exponencial complexa é $\mathbb{C}^{*}$.

(i) As funções $\cos e$ sin são periódicas de período $2 \pi$.

(j) As funções $\cos e$ sin satisfazem

$$
\begin{gathered}
\{z \in \mathbb{C}: \sin (z)=0\}=\pi \mathbb{Z}, \\
\{z \in \mathbb{C}: \cos (z)=0\}=\pi \mathbb{Z}+\frac{\pi}{2} .
\end{gathered}
$$

A inversa da função exponencial real, exp $: \mathbb{R} \rightarrow(0,+\infty)$, é a função logaritmo natural, denotada por $\ln :(0,+\infty) \rightarrow \mathbb{R}$. O número $e$ é o único que satisfaz $\ln (e)=1$.

A seguir estudaremos as inversas locais da função exponencial. O Teorema da Função Inversa Local (p. 39) garante que tais inversas existem, pois $\exp ^{\prime}(z) \neq 0$, para todo $z \in \mathbb{C}$.

Sejam $D_{0}=\mathbb{C} \backslash(-\infty, 0]$ e arctan a inversa da função $\frac{\sin }{\cos }$ restrita ao intervalo $\left(\frac{-\pi}{2}, \frac{\pi}{2}\right)$.

Proposição 4.5. Seja Arg $: D_{0} \rightarrow \mathbb{C}$ a função definida por

$$
\operatorname{Arg}(z)=2 \arctan \left(\frac{\operatorname{Im}(z)}{\operatorname{Re}(z)+|z|}\right), \text { para todo } z \in D_{0} .
$$

A função Arg é contínua em $D_{0}$ e $\operatorname{Arg}\left(D_{0}\right)=(-\pi, \pi)$. Ainda mais,

$$
z=|z| e^{i \operatorname{Arg}(z)}, \text { para todo } z \in D_{0} .
$$

Fixado $k \in \mathbb{Z}$, denotamos $B_{k}=\{z \in \mathbb{C}:(2 k-1) \pi<\operatorname{Im}(z)<(2 k+1) \pi\}$.

Logo, 
(a) $\exp : B_{k} \rightarrow \mathbb{C}$ é injetora

(b) $\exp \left(B_{k}\right)=D_{0}$.

Decorre do comentário acima e do Teorema da Função Inversa (global) que para cada $k \in \mathbb{Z}$, $\left.\exp \right|_{B_{k}}: B_{k} \rightarrow D_{0}$ é um isomorfismo analítico.

Consideremos o aberto $D_{0}$ e seja $f: D_{0} \rightarrow \mathbb{C}$ dada por $f(z)=\ln |z|+i \operatorname{Arg}(z)$, para todo $z \in D_{0}$. Então, $f\left(D_{0}\right) \subset B_{0}$ e $\exp (f(z))=z$, para todo $z \in D_{0}$. Logo, $f=\left(\left.\exp \right|_{B_{0}}\right)^{-1}$ e $f$ é analítica com

$$
f^{\prime}(z)=\frac{1}{z}, \text { para todo } z \in D_{0}
$$

Denotamos a função $f$ por Log e é dita o logaritmo principal (em $\left.D_{0}\right)$ ou ramo principal do logaritmo.

Dado $z \in B(0 ; 1)$, segue da série de Taylor que

$$
\log (1+z)=\sum_{n=1}^{\infty} \frac{(-1)^{n-1}}{n} z^{n} .
$$

Proposição 4.6. Seja $g \in \mathcal{C}\left(D_{0}\right)$. Então, são equivalentes as afirmações a seguir.

(a) $\exp (g(z))=z$, para todo $z \in D_{0}$.

(b) Existe $k \in \mathbb{Z}$ tal que, para todo $z \in D_{0}$

$$
g(z)=\log (z)+2 k i \pi
$$

Para cada $k \in \mathbb{Z}$, denotamos $\log _{k}=\log +2 k i \pi$. Segue então que $\log _{k}=\left(\left.\exp \right|_{B_{k}}\right)^{-1}$.

Proposição 4.7. Sejam $\theta \in[0,2 \pi)$ e $D_{\theta}=e^{i \theta} D_{0}$. Então, para cada $k \in \mathbb{Z}$, a função

$$
\log _{k, \theta}: z \in D_{\theta} \mapsto i \theta+\log _{k}\left(e^{-i \theta} z\right)
$$

satisfaz $\exp \left(\log _{k, \theta}(z)\right)=z$, para todo $z \in D_{\theta}$. E ainda, $\log _{k, \theta}\left(D_{\theta}\right)=B_{k}+i \theta$.

Se $k=0, \log _{k, \theta}$ é dito o logaritmo principal definido em $D_{\theta}$ e o denotamos por $\log _{\theta}$.

Finalizamos esta seção com o seguinte resultado que será útil no capítulo 5 .

Proposição 4.8. Seja $w \in \mathbb{C}$ não nulo. Então, existe uma função contínua $F_{w}: B(w ;|w|) \rightarrow \mathbb{C}$ tal que

$$
\exp \left(F_{w}(z)\right)=z \text {, para todo } z \in B(w ;|w|) .
$$

Demonstração. Seja $\zeta \in \mathbb{C}$ tal que $e^{\zeta}=w$. Basta então considerarmos $F_{w}(z)=\zeta+\log \left(\frac{z}{w}\right)$, onde $z \in B(w ;|w|)$.

\subsection{O índice de um caminho fechado}

Esta seção estabelece algumas noções básicas de topologia, entre elas, os conceitos de homotopia, de espaços conexos por caminhos e espaços simplemente conexos. Nesta seção definiremos também o índice de um caminho fechado (contínuo) e não exigiremos que o caminho seja de classe $\mathcal{C}^{1}$. Seguiremos a abordagem dada em [11]. 
Trataremos apenas do caso particular do plano complexo. Uma abordagem mais geral para espaços topológicos quaisquer pode ser vista em [11].

As definições a seguir são fundamentais para o entendimento deste capítulo.

No que segue, o intervalo $[0,1]$ será denotado por $I, X$ será um subconjunto qualquer de $\mathbb{C}$ e $U$ um subconjunto aberto de $\mathbb{C}$.

Uma função contínua $\gamma:[a, b] \rightarrow X$ será chamada de caminho em $X$. Por simplicidade, quando estiver claro qual o contradomínio da aplicação, nos referimos a $\gamma:[a, b] \rightarrow X$ apenas como caminho. Se $\gamma(a)=\gamma(b)$ o caminho é dito fechado. Os pontos $\gamma(a)$ e $\gamma(b)$ são chamados de ponto inicial e final, respectivamente, ou de extremos de $\gamma$.

Observemos que a imagem de um caminho é um conjunto compacto de $\mathbb{C}$ e, portanto, fechado e limitado.

Definição 4.9. Um conjunto $X$ é dito conexo por caminhos se para quaisquer $p, q \in X$, existe um caminho $\alpha: I \rightarrow X$ satisfazendo $\alpha(0)=p$ e $\alpha(1)=q$. Dizemos que $\alpha$ é um caminho unindo $p$ a $q$.

Proposição 4.10. Um aberto de $\mathbb{C}$ é conexo se e somente se é conexo por caminhos.

Demonstração. Ver [10].

Definiremos a seguir a noção de homotopia.

Definição 4.11. Dois caminhos $\gamma_{1}, \gamma_{2}:[a, b] \rightarrow X$ fechados são $X$-homotópicos como caminhos fechados se existe uma função contínua $H:[a, b] \times I \rightarrow X$ tal que

$$
\left\{\begin{array}{l}
H(x, 0)=\gamma_{1}(x) \\
H(x, 1)=\gamma_{2}(x),
\end{array}\right.
$$

para todo $x$ em $[a, b]$ e, $H(a, t)=H(b, t)$, para todo $t \in I$. Se $\gamma_{2}$ é constante, dizemos que $\gamma_{1} e ́$ homotópico a um ponto de $X$.

A função $H$ é chamada de homotopia entre $\gamma_{1}$ e $\gamma_{2}$ em $X$.

A última igualdade significa que, para todo $t \in I$, o caminho $H_{t}:[a, b] \rightarrow X$ dado por $H_{t}(x)=$ $H(x, t)$, para todo $x \in[a, b]$, é fechado.

Observemos que se $p \in \mathbb{C}$ e $\gamma_{1}, \gamma_{2}:[a, b] \rightarrow \mathbb{C}$ são tais que o segmento $\left[\gamma_{1}(x), \gamma_{2}(x)\right]$ está contido em $\mathbb{C} \backslash\{p\}$ para todo $x \in[a, b]$, então $\gamma_{1}$ e $\gamma_{2}$ são $(\mathbb{C} \backslash\{p\})$-homotópicos como caminhos fechados. De fato, temos que

$$
H(x, t)=(1-t) \gamma_{1}(x)+t \gamma_{2}(x), \text { onde }(x, t) \in[a, b] \times I,
$$

é uma homotopia entre $\gamma_{1}$ e $\gamma_{2}(\mathrm{em}(\mathbb{C} \backslash\{p\}))$.

Uma consequência importante da observação anterior é dada a seguir.

Proposição 4.12 (Poincaré-Bohl). Sejam $\gamma_{1}, \gamma_{2}:[a, b] \rightarrow \mathbb{C}$ dois caminhos fechados satisfazendo

$$
\left|\gamma_{1}(x)-\gamma_{2}(x)\right|<\left|\gamma_{1}(x)-p\right|, \quad \text { para todo } x \in[a, b]
$$

Então, $\gamma_{1}$ e $\gamma_{2}$ são $(\mathbb{C} \backslash\{p\})$-homotópicos como caminhos fechados. 
Demonstração. Observemos que se $p \in\left[\gamma_{1}\left(x_{0}\right), \gamma_{2}\left(x_{0}\right)\right]$ para algum $x_{0} \in[a, b]$, então

$$
\left|\gamma_{1}\left(x_{0}\right)-\gamma_{2}\left(x_{0}\right)\right|=\left|\gamma_{1}\left(x_{0}\right)-p\right|+\left|\gamma_{2}\left(x_{0}\right)-p\right| \geq\left|\gamma_{1}\left(x_{0}\right)-p\right| .
$$

Logo,

$$
H(x, t)=(1-t) \gamma_{1}(x)+t \gamma_{2}(x), \text { onde }(x, t) \in[a, b] \times I,
$$

é uma homotopia entre $\gamma_{1}$ e $\gamma_{2}$.

Definição 4.13. Dizemos que um aberto $\Omega$ é simplesmente conexo se é conexo e todo caminho fechado em $\Omega$ é homotópico a um ponto de $\Omega$.

Decorre da proposição 4.10 que um aberto $\Omega$ simplesmente conexo é também conexo por caminhos.

Seja $\xi: \mathbb{R} \rightarrow S^{1}$ a função dada por $\xi(t)=e^{i t}$, para todo $t \in \mathbb{R}$. Temos que $\xi$ é contínua, ainda mais, lembrando que $\left.\xi\right|_{[0,2 \pi]}:[0,2 \pi] \rightarrow S^{1}$ é sobrejetora, como consequência temos a proposição abaixo.

Proposição 4.14. Valem as seguintes propriedades

(a) $\xi$ é aberta.

(b) A restrição de $\xi$ aos intervalos abertos da forma $(t, t+2 \pi)$ é um homeomorfismo sobre $S^{1} \backslash\{\xi(t)\}$.

Demonstração. (a) Seja $V \subset \mathbb{R}$ aberto. É suficiente mostrarmos que o conjunto $F=S^{1} \backslash \xi(V)$ é fechado em $S^{1}$. Observemos que

$$
\xi^{-1}(\xi(V))=\bigcup_{n \in \mathbb{Z}}(V+2 n \pi)
$$

Com efeito, temos $\xi(x)=1$ se e somente se $x \in 2 \pi \mathbb{Z}$.

Ainda, $\bigcup_{n \in \mathbb{Z}} V+2 n \pi$ é aberto em $\mathbb{R}$, portanto, seu complementar $\xi^{-1}(F)$ é fechado em $\mathbb{R}$.

Temos que $\xi\left(\xi^{-1}(F) \cap[0,2 \pi]\right) \subset F$.

Ainda, como $\left.\xi\right|_{[0,2 \pi]}$ é sobrejetora sobre $S^{1}$, dado $y \in F$, existe $x \in[0,2 \pi]$ tal que $y=\xi(x)$.

Portanto,

$$
F=\xi\left(\xi^{-1}(F) \cap[0,2 \pi]\right) .
$$

Como $\xi^{-1}(F) \cap[0,2 \pi]$ é compacto, pela continuidade de $\xi$, temos $F$ compacto e, portanto, fechado em $S^{1}$.

(b) Fixemos $t \in \mathbb{R}$. É claro que $\left.\xi\right|_{(t, t+2 \pi)}$ é uma bijeção contínua sobre $S^{1} \backslash\{\xi(t)\}$. Pelo item $(a)$, segue que $\left.\xi\right|_{(t, t+2 \pi)}$ é aberta. Logo, sua inversa é contínua.

Dadas as funções $f_{k}:\left[t_{k-1}, t_{k}\right] \rightarrow \mathbb{R}$, onde $k=1,2, \ldots, m$, definimos a justaposição de tais funções, denotada por $\left(f_{1} \vee f_{2} \vee \ldots \vee f_{m}\right)$, como segue

$$
\left(f_{1} \vee f_{2} \vee \ldots \vee f_{m}\right)(t)=f_{k}(t), \text { se } t \in\left[t_{k-1}, t_{k}\right]
$$


Teorema 4.15. Sejam $\gamma:[a, b] \rightarrow S^{1}$ contínua e $\theta_{0}$ um número real tal que $\gamma(a)=e^{i \theta_{0}}$. Então, existe uma única função contínua $\theta:[a, b] \rightarrow \mathbb{R}$ tal que

$$
\gamma(t)=e^{i \theta(t)}, \quad \text { para todo } t \in[a, b] \text { e } \theta(a)=\theta_{0} .
$$

Demonstração. Como $\gamma$ é contínua e $[a, b]$ é compacto, segue que $\gamma$ é uniformemente contínua. Portanto, existe $\delta>0$ tal que

$$
|\gamma(t)-\gamma(s)|<1, \text { para todos } s, t \in[a, b] \operatorname{com}|s-t|<\delta
$$

Seja $m$ um inteiro tal que $m>\frac{|b-a|}{\delta}$ e $t_{k}=a+k \frac{(b-a)}{m}$ para $k=0,1, \ldots, m$.

Fixado $k=1,2, \ldots, m$, temos $\left|\gamma(t)-\gamma\left(t_{k}\right)\right|<1$, para todo $t \in\left[t_{k}, t_{k+1}\right]$. Portanto, segue que

$$
\gamma\left(\left[t_{k-1}, t_{k}\right]\right) \neq S^{1}
$$

Logo, existem $\alpha \in \mathbb{R}$ e $p \in S^{1}$ tais que $p=e^{i \alpha}$ e

$$
\gamma\left(I_{1}\right) \subset S^{1} \backslash\{p\}
$$

Denotando $\psi=\left.\xi\right|_{(\alpha, \alpha+2 \pi)}$, pela proposição 4.14 temos que $\psi:(\alpha, \alpha+2 \pi) \rightarrow S^{1} \backslash\{p\}$ é um homeomorfismo.

Definamos

$$
\theta_{1}(t): t \in\left[t_{0}, t_{1}\right] \mapsto \psi^{-1}(\gamma(t))+\theta_{0}-\psi^{-1}(\gamma(a)) .
$$

A função $\theta_{1}:\left[t_{0}, t_{1}\right] \rightarrow \mathbb{R}$ é contínua, $\theta_{1}(a)=t_{0}$ e $\gamma(t)=e^{i \theta_{1}(t)}$, para todo $t \in\left[t_{0}, t_{1}\right]$.

Procedendo analogamente, obtemos $\theta_{2}:\left[t_{1}, t_{2}\right] \rightarrow \mathbb{R}$ contínua e tal que

$$
\theta_{2}\left(t_{1}\right)=\theta_{1}\left(t_{1}\right) \text { e } \gamma(t)=e^{i \theta_{2}(t)} \text {, para todo } t \in\left[t_{1}, t_{2}\right]
$$

Repetindo tal procedimento, determinamos $m$ funções $\theta_{k}:\left[t_{k}, t_{k+1}\right] \rightarrow \mathbb{R}$ contínuas, onde $k=1,2, \ldots, m$, satisfazendo

$$
\theta_{k+1}\left(t_{k}\right)=\theta_{k}\left(t_{k}\right) \text { e } \gamma(t)=e^{i \theta_{k}(t)}, \text { para todo } t \in\left[t_{k}, t_{k+1}\right]
$$

Para finalizarmos, basta notarmos que a justaposição $\theta=\left(\theta_{1} \vee \theta_{2} \vee \ldots \vee \theta_{m}\right)$ é a função procurada.

Quanto à unicidade de $\theta$, observemos que se $\theta_{1}$ e $\theta_{2}$ são duas funções contínuas tais que $e^{i \theta_{1}(t)}=$ $e^{i \theta_{2}(t)}$, para todo $t \in[a, b]$, então existe um inteiro $k$ tal que $\theta_{2}=\theta_{1}+2 k \pi$ (os conexos de $\mathbb{Z}$ são os conjuntos unitários). Como $\theta_{1}(a)=\theta_{2}(a)$, segue que $\theta_{1}=\theta_{2}$.

Definição 4.16. Seja $f: X \rightarrow \mathbb{C}^{*}$ contínua. Se $\theta: X \rightarrow \mathbb{R}$ é uma função contínua satisfazendo $f(z)=|f(z)| e^{i \theta(z)}$, para todo $z \in X$, dizemos que $\theta$ é um argumento de $f$ em $X$.

Pelo que vimos, todo caminho $\gamma$ tal que $\operatorname{Imagem}(\gamma) \subset \mathbb{C}^{*}$ tem um argumento.

Consideremos a seguir $p \in \mathbb{C}$ e um caminho fechado $\gamma:[a, b] \rightarrow \mathbb{C} \backslash\{p\}$. Então, pelo teorema 4.15 , existe $\theta:[a, b] \rightarrow \mathbb{R}$ contínua tal que $\gamma(t)=p+|\gamma(t)-p| e^{i \theta(t)}$, para todo $t \in[a, b]$. 
Sendo $\gamma$ um caminho fechado, decorre que

$$
\frac{\theta(b)-\theta(a)}{2 \pi} \in \mathbb{Z}
$$

Ainda mais, dois argumentos distintos de uma função contínua definida num conexo diferem por uma constante. Isto nos leva a seguinte definição.

Definição 4.17. O indice de um caminho fechado $\gamma:[a, b] \rightarrow \mathbb{C} \backslash\{p\}$ em relação ao ponto $p$ é o número inteiro

$$
\operatorname{Ind}(\gamma, p)=\frac{\theta(b)-\theta(a)}{2 \pi},
$$

onde $\theta$ é um argumento em $[a, b]$ do caminho $c: t \in[a, b] \mapsto \gamma(t)-p$.

As afirmações da observação a seguir decorrem trivialmente da definição de índice e serão importantes para a demonstração do Princípio do Argumento.

Observação 4.18. Sejam $\gamma$ um caminho fechado e $p$ um ponto em $\mathbb{C} \backslash \operatorname{Imagem}(\gamma)$. Valem as seguintes propriedades.

(a) $\operatorname{Ind}(\gamma-p, 0)=\operatorname{Ind}(\gamma, p)$.

(b) Se $\gamma: t \in[a, b] \mapsto p+\gamma_{1}(t) \gamma_{2}(t) \ldots \gamma_{n}(t)$, onde $\gamma_{k}$ é um caminho fechado definido em $[a, b]$, temos que 0 não pertence a imagem de $\gamma_{k}$, para todo $k=1,2, \ldots, n$. Considere $\theta_{k}$ um argumento de $\gamma_{k}$ em $[a, b], k=1,2, \ldots, n$. Então,

$$
\gamma(t)=p+|\gamma(t)-p| e^{i\left(\theta_{1}(t)+\theta_{2}(t)+\cdots+\theta_{n}(t)\right)}
$$

e segue que

$$
\operatorname{Ind}(\gamma, p)=\sum_{k=0}^{n} \operatorname{Ind}\left(\gamma_{k}, 0\right) .
$$

(c) Se $m \in \mathbb{N}$ e $\alpha: t \in[a, b] \mapsto(\gamma(t)-p)^{m}$ é um caminho fechado, então decorre dos itens anteriores que

$$
\operatorname{Ind}(\alpha, 0)=m \operatorname{Ind}(\gamma-p, 0)=m \operatorname{Ind}(\gamma, p) .
$$

A proposição 4.19 lista mais algumas das propriedades do índice de um caminho fechado.

Proposição 4.19. (a) Sejam $\gamma_{1}, \gamma_{2}:[a, b] \rightarrow \mathbb{C}$ dois caminhos fechados satisfazendo

$$
\left|\gamma_{2}(t)-\gamma_{1}(t)\right|<\left|\gamma_{1}(t)-p\right|, \quad \text { para todo } t \in[a, b]
$$

Então,

$$
\operatorname{Ind}\left(\gamma_{1}, p\right)=\operatorname{Ind}\left(\gamma_{2}, p\right) .
$$

(b) Para todos $k \in \mathbb{Z}, r>0$ e p $\in \mathbb{C}$, o caminho fechado $\gamma:[0,2 \pi] \rightarrow \mathbb{C}$, dado por $\gamma(t)=p+r e^{i k t}$, satisfaz $\operatorname{Ind}(\gamma, p)=k$.

Demonstração. (a) Da desigualdade em $(a)$ segue que $p$ não pertence à união $\operatorname{Imagem}\left(\gamma_{1}\right) \cup$ $\operatorname{Imagem}\left(\gamma_{2}\right)$.

Sejam

$$
\Gamma: t \in[a, b] \mapsto \frac{\gamma_{2}(t)-\gamma_{1}(t)}{\gamma_{1}(t)-p} \text { e } \Omega=\{z \in \mathbb{C}: \operatorname{Re}(z)>0\}
$$


Segue que $|\Gamma(t)|<1$, para todo $t \in[a, b]$.

Logo,

$$
\Gamma(t)+1 \in \Omega, \text { para todo } t \in[a, b] .
$$

Consideremos a função contínua

$$
\theta: z \in \Omega \mapsto \arcsin \left[\operatorname{Im}\left(\frac{z}{|z|}\right)\right] \in\left(\frac{-\pi}{2}, \frac{\pi}{2}\right) .
$$

Logo, escrevendo $\Gamma(t)+1=|\Gamma(t)+1| e^{i \theta(\Gamma(t)+1)}$, para todo $t \in[a, b]$, temos que

$$
2 \pi \operatorname{Ind}(\Gamma+1,0)=\theta(\Gamma(b)+1)-\theta(\Gamma(a)+1)=0 .
$$

Temos também que $\gamma_{2}(t)-p=[\Gamma(t)+1]\left[\gamma_{1}(t)-p\right]$.

Consequentemente

$$
\operatorname{Ind}\left(\gamma_{2}, p\right)=\operatorname{Ind}(\Gamma+1,0)+\operatorname{Ind}\left(\gamma_{1}, p\right)=\operatorname{Ind}\left(\gamma_{1}, p\right)
$$

(b) É trivial verificar.

Proposição 4.20. Sejam $\Omega$ aberto, $\gamma_{1}, \gamma_{2}:[a, b] \rightarrow \Omega$ caminhos fechados e $H$ uma $\Omega$ - homotopia entre $\gamma_{1}$ e $\gamma_{2}$. Para cada $t \in[0,1]$ definamos $H_{t}: x \in[a, b] \mapsto H(x, t)$. Se $\alpha \in \mathbb{C} \backslash \operatorname{Imagem}(H)$, então a função Ind $\left(H_{t}, \alpha\right)$, onde $t \in[0,1]$, é constante.

Demonstração. Seja $t_{0} \in[0,1]$ e $d=d(\alpha, \operatorname{Imagem}(H))$. Como $H$ é uniformemente contínua, existe $\delta>0$ tal que se $\left|t-t_{0}\right|<\delta$ e $t \in[0,1]$, então

$$
\left|H_{t}(x)-H_{t_{0}}(x)\right|<1 \text {, para todo } x \in[a, b] .
$$

Decorre da proposição 4.19 que

$$
\operatorname{Ind}\left(H_{t}, \alpha\right)=\operatorname{Ind}\left(H_{t_{0}}, \alpha\right) \text {, se }\left|t-t_{0}\right|<\delta \text {. }
$$

Portanto, $\operatorname{Ind}\left(H_{t}, \alpha\right)$, onde $t \in[0,1]$, é uma função localmente constante definida num conexo. Logo, constante.

Dado um caminho fechado $\gamma$, definimos $\operatorname{Ind}_{\gamma}: \mathbb{C} \backslash \operatorname{Imagem}(\gamma) \rightarrow \mathbb{Z}$ por $\operatorname{Ind}_{\gamma}(w)=\operatorname{Ind}(\gamma, w)$, para todo $w \in \mathbb{C} \backslash \operatorname{Imagem}(\gamma)$. Na proposição 4.21 veremos algumas propriedades da função $\operatorname{Ind}_{\gamma}$.

Proposição 4.21. Sejam $\gamma:[a, b] \rightarrow \mathbb{C}$ um caminho fechado e $W=\mathbb{C} \backslash$ Imagem $(\gamma)$. Então, valem as afirmações abaixo.

(a) A função Ind é contínua em W.

(b) A função Ind $d_{\gamma}$ é constante em cada componente conexa de $W$.

Demonstração. Sejam $w \in W$ e $r>0$ tal que $|\gamma(t)-w| \geq r$, para todo $t \in[a, b]$.

Mostraremos que $\operatorname{Ind}_{\gamma}$ é constante $B(w ; r)$. 
Consideremos $w_{1} \in B(w ; r)$. Logo, $w_{1} \in W$.

Seja $\gamma_{1}:[a, b] \rightarrow \mathbb{C}$ dada por $\gamma_{1}(t)=\gamma(t)+w-w_{1}$. Então,

$$
\left|\gamma_{1}(t)-\gamma(t)\right|<r \leq|\gamma(t)-w|, \text { para todo } t \in[a, b]
$$

Segue da proposição 4.19 que $\operatorname{Ind}(\gamma, w)=\operatorname{Ind}\left(\gamma_{1}, w\right)$.

Observemos que $\gamma_{1}(t)-w=\gamma(t)-w_{1}$ para todo $t \in[a, b]$. Logo, pela definição 4.17 temos que $\operatorname{Ind}\left(\gamma, w_{1}\right)=\operatorname{Ind}\left(\gamma_{1}, w\right)$.

Portanto,

$$
\operatorname{Ind}_{\gamma}(w)=\operatorname{Ind}_{\gamma}\left(w_{1}\right) \text {, para todo } w_{1} \in B(w ; r) .
$$

Como $w$ é arbitrário, segue que $\operatorname{Ind}_{\gamma}$ é contínua em $W$.

Dado que os conexos de $\mathbb{Z}$ são os conjuntos unitários, concluímos que a função $\operatorname{Ind}_{\gamma}$ é constante em cada componente conexa de $W$.

Antes de prosseguirmos, faremos mais algumas observações.

Observação 4.22. Seja $\gamma:[a, b] \rightarrow \mathbb{C}$ um caminho fechado e $r>0$ tal que $|\gamma(t)| \leq r$, para todo $t \in[a, b]$. Se $w \in \mathbb{C}$ satisfaz $|w|>r$, então $\operatorname{Ind}(\gamma, w)=0$. Com efeito, seja $\gamma_{1}:[a, b] \rightarrow \mathbb{C}$ dada por $\gamma_{1}(t)=0$, para todo $t \in[a, b]$, assim

$$
\left|\gamma(t)-\gamma_{1}(t)\right|=|\gamma(t)| \leq r<|w|=|0-w|=\left|\gamma_{1}(t)-w\right|, \text { para todo } t \in[a, b] .
$$

$\operatorname{Logo}, \operatorname{Ind}(\gamma, w)=\operatorname{Ind}\left(\gamma_{1}, w\right)=0$.

Segue que Ind $d_{\gamma}$ é nula na única componente conexa de $\mathbb{C} \backslash \operatorname{Imagem}(\gamma)$ que contém $\mathbb{C} \backslash D(0 ; r)$. Ainda, as demais componentes conexas de $\mathbb{C} \backslash \operatorname{Imagem}(\gamma)$ estão contidas em $D(0 ; r)$ e são limitadas.

Definição 4.23. Seja $\gamma$ um caminho fechado e $p \in \mathbb{C}$. Dizemos que

(i) $p$ é interior a $\gamma$ se $p \in \mathbb{C} \backslash \operatorname{Imagem}(\gamma)$ e $\operatorname{Ind}(\gamma, p) \neq 0$.

(ii) p está em $\gamma$ se $p \in \operatorname{Imagem}(\gamma)$.

(iii) $p$ é exterior a $\gamma$ se $p \in \mathbb{C} \backslash \operatorname{Imagem}(\gamma)$ e $\operatorname{Ind}(\gamma, p)=0$.

Se $\operatorname{Int}(\gamma)$ e $\operatorname{Ext}(\gamma)$ denotam o interior e o exterior de $\gamma$, respectivamente, temos a seguinte decomposição em conjuntos dois a dois disjuntos

$$
\mathbb{C}=\operatorname{Imagem}(\gamma) \cup \operatorname{Int}(\gamma) \cup \operatorname{Ext}(\gamma)
$$

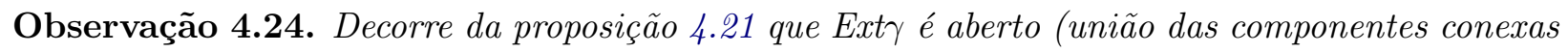
abertas de $\mathbb{C} \backslash \operatorname{Imagem}(\gamma)$ em que o índice é nulo) e, pela observação 4.22, contém o complementar de uma bola fechada. Logo, $\operatorname{Int}(\gamma) \cup \operatorname{Imagem}(\gamma)$ é fechado e limitado e, portanto, compacto.

A seguir, como aplicação do índice de um caminho fechado, demonstraremos o Princípio do Argumento, o Teorema de Rouché e a existência do logaritmo de uma função contínua. 


\subsection{O Princípio do Argumento e o Teorema de Rouché}

Iniciamos esta seção com uma versão do Princípio do Argumento com uma prova simples. Em [11] é dada uma versão desse resultado para polinômios, nós apenas o generalizamos.

Antes, recordemos o Princípio dos zeros isolados. Sejam $\Omega$ um aberto conexo e uma função $f \in \mathcal{A}(\Omega)$ não nula e $a$ um zero de $f$. Então, existem um número natural $m \geq 1$ e $g \in \mathcal{A}(\Omega)$ tais que $f(z)=(z-a)^{m} g(z)$, para todo $z \in \Omega$, com $g(a) \neq 0$. Dizemos que $a$ é um zero de ordem $m$ de $f$ e denotamos $m=\nu(f ; a)$. Caso $w \in \Omega$ e $f(w) \neq 0$, escrevemos $\nu(f ; w)=0$

Se $\Omega$ é um aberto conexo, $f \in \mathcal{A}(\Omega)$ e $z_{1}, z_{2}, \ldots, z_{n}$ são todos os zeros de $f$ em $A \subset \Omega$, como consequência do Princípio do zeros isolados, temos que existe $g \in \mathcal{A}(\Omega)$ e sem zeros em $A$ tal que

$$
f(z)=\left(z-z_{1}\right)^{\nu\left(f ; z_{1}\right)}\left(z-z_{2}\right)^{\nu\left(f ; z_{2}\right)} \cdots\left(z-z_{n}\right)^{\nu\left(f ; z_{n}\right)} g(z) .
$$

Proposição 4.25 (Contagem de Zeros). Sejam $\Omega$ aberto conexo de $\mathbb{C}$, uma função $f \in \mathcal{A}(\Omega)$ não constante e $a \in \Omega$ um zero de $f$. Então, existe $R>0$ tal que para todo $0<r<R$, se $\gamma(t)=a+r e^{i t}$, onde $0 \leq t \leq 2 \pi$, temos $\operatorname{Ind}(f \circ \gamma, 0)=\nu(f ; a)$.

Demonstração. Sendo $f$ analítica não constante e $\Omega$ aberto conexo, existem um natural $k \geq 1 \mathrm{e}$ $g \in \mathcal{A}(\Omega)$ tais que $f(z)=(z-a)^{k} g(z)$, para todo $z \in \Omega$ e $g(a) \neq 0$.

Seja $b=g(a)$. Como $b \neq 0$, podemos escrever $g(z)=b+b G(z)$, com $G$ analítica em $\Omega$ e $G(a)=0$. Pela continuidade de $G$, podemos fixar $R>0$ tal que $|G(z)|<1$, se $|z-a|<R$.

Então, se $0<|z-a|<R$, segue que

$$
f(z)=(z-a)^{k} g(z)=b(z-a)^{k}+b G(z)(z-a)^{k},
$$

com

$$
\left|b G(z)(z-a)^{k}\right|<\left|b(z-a)^{k}\right| .
$$

Fixemos $0<r<R$. Considerando o caminho fechado dado por $\alpha: t \in[0,2 \pi] \mapsto b r^{k} e^{i k t}$ e a função inteira $h: z \in \mathbb{C} \mapsto b(z-a)^{k}$, temos que $\alpha(t)=(h \circ \gamma)(t)$, para todo $t \in[0,2 \pi]$.

Pela desigualdade em 4.1, segue que para todo $t \in[0,2 \pi]$

$$
|f(\gamma(t))-\alpha(t)|<|\alpha(t)|
$$

Pela proposição 4.19, temos que

$$
\operatorname{Ind}(f \circ \gamma, 0)=\operatorname{Ind}(\alpha, 0)=k=\nu(f ; a) .
$$

Dada uma função $f$ definida em $\Omega$, denotamos o conjunto dos seus zeros por $Z(f)$, isto é, $Z(f)=\{a \in \Omega: f(a)=0\}$.

Definição 4.26. Sejam $\Omega$ um aberto conexo, uma função $f \in \mathcal{A}(\Omega)$ não constante e $\gamma:[a, b] \rightarrow \Omega$ um caminho fechado homotópico a um ponto de $\Omega$ tal que $\operatorname{Ind}(\gamma, p)=1$, se $p \in \operatorname{Int}(\gamma)$. O número 
de zeros de $f$ no interior de $\gamma$ e contados com sua multiplicidade é dado por

$$
Z(f ; \gamma)=\sum_{a \in Z(f) \cap \operatorname{Int\gamma }} \nu(f ; a)
$$

Observemos que a soma acima é finita, pois $\operatorname{Int}(\gamma) \cup \operatorname{Imagem}(\gamma)$ é um compacto de $\Omega$.

Analisando a definição anterior e a proposição 4.25, surge a pergunta natural : é possível contar zeros de funções analíticas em certos conjuntos utilizando o índice de um determinado caminho em relação ao zero? A resposta é sim e veremos sua prova no Princípio do Argumento. Com o intuito de justificar o porquê consideraremos que o caminho seja homotópico a um ponto, façamos a seguinte observação. Mantida a notação da proposição anterior, temos que

$$
\operatorname{Ind}(f \circ \gamma, 0)=\operatorname{Ind}\left(b^{-1} \alpha, 0\right)+\operatorname{Ind}(g \circ \gamma, 0)=\operatorname{Ind}(\alpha, 0)+\operatorname{Ind}(g \circ \gamma, 0) .
$$

Mas obtivemos na demonstração da proposição 4.25 que

$$
\operatorname{Ind}(\alpha, 0)=\operatorname{Ind}(f \circ \gamma, 0)
$$

Portanto, $\operatorname{Ind}(g \circ \gamma, 0)=0$, o que nos induz a pensar que tal condição será útil.

A seguir demonstraremos o Princípio do Argumento. Essencialmente nossa prova se reduz a encontrar um compacto $K$ contido no domínio da função analítica $f$, para então obter uma quantidade finita de zeros de $f$ em $K$ e, assim, utilizarmos a fatoração citada no início desta seção. Enfatizamos que nossa versão é livre de integração e não exige que o caminho considerado seja de classe $\mathcal{C}^{1}$ e, portanto, tal exigência feita nas versões mais conhecidas do Princípio do Argumento e, consequentemente do Teorema de Rouché, é desnecessária.

Antes, notemos que se $\gamma$ é um caminho fechado e $H$ é uma $\Omega$-homotopia entre $\gamma$ e um ponto $\alpha$, então, pela proposição 4.20 , segue que para todo $p \in \mathbb{C} \backslash \Omega$

$$
\operatorname{Ind}(\gamma, p)=\operatorname{Ind}\left(H_{0}, p\right)=\operatorname{Ind}\left(H_{1}, p\right)=\operatorname{Ind}(\alpha, p)=0 .
$$

Teorema 4.27 (Princípio do Argumento). Sejam $\Omega$ um aberto conexo, $\gamma:[a, b] \rightarrow \Omega$ um caminho fechado homotópico a um ponto de $\Omega$, uma função $f \in \mathcal{A}(\Omega)$ não constante e sem zeros na $\operatorname{Imagem}(\gamma)$. Nestas condições, vale o seguinte

$$
\operatorname{Ind}(f \circ \gamma, 0)=\sum_{a \in Z(f)} \nu(f ; a) \operatorname{Ind}(\gamma ; a) .
$$

Demonstração. Seja $H$ uma homotopia entre $\gamma$ e um ponto $a$ em $\Omega$. Pelo comentário acima, temos que $\operatorname{Ind}(\gamma, p)=0$, para todo $p \in \mathbb{C} \backslash \Omega$. Logo, $\operatorname{Int}(\gamma) \subset \Omega$.

Decorre da observação 4.24 e da continuidade de $H$ que $K=\operatorname{Int}(\gamma) \cup \operatorname{Imagem}(\gamma) \cup \operatorname{Imagem}(H)$ é um compacto contido em $\Omega$. Pelo Princípio dos zeros isolados, $f$ tem uma quantidade finita $z_{1}, z_{2}, \ldots, z_{n}$ de zeros em $K$.

Ainda, podemos escrever a seguinte fatoração para $f$

$$
f(z)=\left(z-z_{1}\right)^{\nu\left(f ; z_{1}\right)}\left(z-z_{2}\right)^{\nu\left(f ; z_{2}\right)} \cdots\left(z-z_{n}\right)^{\nu\left(f ; z_{n}\right)} h(z),
$$


com $h$ analítica (em $\Omega$ ) e sem zeros em $K$.

Como $K^{c} \subset$ Ext $\gamma$, valem as igualdades

$$
\sum_{a \in Z(f)} \nu(f ; a) \operatorname{Ind}(\gamma ; a)=\sum_{a \in Z(f) \cap K} \nu(f ; a) \operatorname{Ind}(\gamma ; a)+\sum_{a \in Z(f) \cap K^{c}} \nu(f ; a) \operatorname{Ind}(\gamma ; a)=\sum_{k=1}^{n} \nu\left(f ; z_{k}\right) \operatorname{Ind}\left(\gamma ; z_{k}\right) .
$$

Aplicando a observação 4.18, tem-se que

$$
\operatorname{Ind}(f \circ \gamma, 0)=\nu\left(f ; z_{1}\right) \operatorname{Ind}\left(\gamma, z_{1}\right)+\cdots+\nu\left(f ; z_{n}\right) \operatorname{Ind}\left(\gamma, z_{n}\right)+\operatorname{Ind}(h \circ \gamma ; 0) .
$$

Resta mostrarmos que $\operatorname{Ind}(h \circ \gamma, 0)$ e a conclusão segue.

Observemos que $h \circ \gamma$ é homotópico $\left(\mathrm{em} \mathbb{C}^{*}\right)$ ao ponto $h(a)$, pois $h$ não se anula em $\operatorname{Imagem}(\gamma)$. Logo, pela proposição 4.19 , temos $\operatorname{Ind}(h \circ \gamma, 0)=0$.

Uma consequência importante do Princípio do Argumento é o conhecido Teorema de Rouché que nos permite comparar a quantidade de zeros de duas funções analíticas.

Teorema 4.28 (Rouché). Sejam $\Omega$ aberto conexo de $\mathbb{C}, f, g \in \mathcal{A}(\Omega)$ funções não constantes e $\gamma$ um caminho fechado homotópico a um ponto de $\Omega$ tal que $\operatorname{Ind}(\gamma, p)=1$, se $p \in \operatorname{Int}(\gamma)$. Suponha que

$$
|f(z)-g(z)|<|g(z)|, \quad \text { para todo } z \in \operatorname{Imagem}(\gamma)
$$

Então, f e g tem o mesmo número de zeros no interior de $\gamma$.

Demonstração. Da desigualdade $|(f \circ \gamma)(t)-(g \circ \gamma)(t)|<|(g \circ \gamma)(t)|$, para todo $t$ no domínio de $\gamma$, temos que $f$ e $g$ não tem zeros em $\operatorname{Imagem}(\gamma)$.

Pela proposição 4.19, temos

$$
\operatorname{Ind}(f \circ \gamma, 0)=\operatorname{Ind}(g \circ \gamma, 0)
$$

Segue do Princípio do Argumento que

$$
\operatorname{Ind}(f \circ \gamma, 0)=\sum_{a \in Z(f)} \nu(f ; a) \operatorname{Ind}(\gamma ; a)=Z(f ; \gamma)
$$

Analogamente temos $\operatorname{Ind}(g \circ \gamma, 0)=Z(g ; \gamma)$. Portanto, $f$ e $g$ tem mesma quantidade de zeros $\operatorname{em} \operatorname{Int}(\gamma)$.

Poderíamos nos perguntar o que aconteceria se uma das funções no Teorema de Rouché fosse apenas contínua. É claro que não seríamos capazes de contar zeros, pois não temos uma noção bem definida da ordem de um zero de uma função contínua. No entanto, se quisermos apenas tirar conclusões sobre a existência de zeros, temos um resultado positivo. O corolário a seguir é uma versão do Teorema de Rouché que exige a analiticidade de apenas uma das funções. É ainda um caso particular do resultado mais geral encontrado em [13]. Tal versão não é usualmente apresentada em livros, porém, nossa demonstração é bastante simples. 
Corolário 4.29 (Rouché). Sejam $\Omega$ aberto conexo de $\mathbb{C}$ e as funções $f \in \mathcal{C}(\Omega)$ e $g \in \mathcal{A}(\Omega)$ não constantes. Suponha que $D(a ; r) \subset \Omega$ e para todo $z \in S(a ; r)$ vale

$$
|f(z)-g(z)|<|g(z)| \text { ou }|f(z)-g(z)|<|f(z)|
$$

Então, se g tem zeros em B(a;r), a função $f$ também se anula.

Demonstração. Consideremos o caminho $\gamma: x \in[0,2 \pi] \mapsto a+r e^{i x}$ e a aplicação $H:[0,2 \pi] \times[0,1] \rightarrow$ $D(a ; r)$ dada por $H(x, t)=a+r t e^{i x}$. Então, $H$ é uma homotopia entre $\gamma$ e o ponto $a$.

Pelo Princípio do Argumento, como $g$ se anula em $B(a ; r)$, temos que $\operatorname{Ind}(g \circ \gamma, 0) \neq 0$.

Suponha que $f$ não tem zeros em $B(a ; r)$. Logo, por hipótese, $f$ não se anula em $D(a ; r)$. Então, $f \circ H$ é uma homotopia entre $f \circ \gamma$ e o ponto $f(a)$ em $\mathbb{C}^{*}$.

Assim,

$$
\operatorname{Ind}(f \circ \gamma, 0)=\operatorname{Ind}(f(a), 0)=0 .
$$

Portanto, segue do Princípio do Argumento e da proposição 4.19 que

$$
Z(g ; \gamma)=\operatorname{Ind}(g \circ \gamma, 0)=\operatorname{Ind}(f \circ \gamma, 0)=0
$$

o que contraria nossa hipótese sobre $g$.

\subsection{O logaritmo de uma função contínua}

A seguir utilizaremos o conceito de índice de um caminho fechado para estudar as funções $f: \Omega \rightarrow \mathbb{C}^{*}$ contínuas, onde $\Omega$ é um aberto simplesmente conexo do plano. Esta seção tem como referência [4].

Definição 4.30. Sejam $U$ um subconjunto aberto de $\mathbb{C}$ e as funções $f: U \rightarrow \mathbb{C}^{*}$ e $g: U \rightarrow \mathbb{C}$ contínuas. Dizemos que $g$ é um logaritmo contínuo de $f$ em $U$ se $f(z)=e^{g(z)}$, para todo $z \in U$.

Observemos que se $f: U \rightarrow \mathbb{C}^{*}$ é contínua, então

(a) Se $g$ é um logaritmo contínuo de $f$, então $\operatorname{Im}(g)$ é um argumento de $f$ em $U$.

(b) Se $\theta$ é um argumento de $f$ em $U$, então a função dada por $\ln |f(z)|+i \theta(z)$, para todo $z \in U$, é um logaritmo contínuo de $f$ em $U$.

Segue que uma função contínua tem um logaritmo contínuo se e somente se tem um argumento.

A seguir mostramos uma condição necessária e suficiente para a existência do logaritmo contínuo de uma dada função contínua num aberto conexo de $\mathbb{C}$. Este resultado pode ser encontrado em [4] e, optamos por demonstrá-lo devido à sua extensão.

Teorema 4.31. Sejam $\Omega$ um aberto conexo de $\mathbb{C}$ e uma função $f: \Omega \rightarrow \mathbb{C}^{*}$ contínua.

São equivalentes as afirmações abaixo.

(a) $\operatorname{Ind}(f \circ \gamma, 0)=0$, para todo $\gamma$ caminho fechado em $\Omega$.

(b) $f$ tem um logaritmo contínuo em $\Omega$. 
Demonstração. $(a \Rightarrow b)$ Fixemos $z_{0} \in \Omega$. Pela proposição $4.10, \Omega$ é conexo por caminhos. Logo, para cada $z \in \Omega$, podemos fixar $\gamma_{z}$ um caminho unindo $z_{0}$ a $z$ em $\Omega$ tal que $\gamma_{z}(0)=z_{0}$ e $\gamma_{z}(1)=z$. Portanto, o caminho $f \circ \gamma_{z}$ tem um logaritmo contínuo $\varphi_{z}$, qualquer que seja $z \in \Omega$. Isto é,

$$
\left(f \circ \gamma_{z}\right)(t)=e^{\varphi_{z}(t)} \text {, para todo } t \in[0,1] .
$$

Definamos a seguinte função

$$
\Phi: z \in \Omega \mapsto \varphi_{z}(1)
$$

Para todo $z \in \Omega$, temos

$$
e^{\Phi(z)}=e^{\varphi_{z}(1)}=\left(f \circ \gamma_{z}\right)(1)=f(z)
$$

Resta mostrarmos que a função $\Phi$ é contínua.

Seja $\zeta \in \Omega$ arbitrário e fixado. Consideremos ainda $r>0$ tal que

$$
B(\zeta ; r) \subset \Omega \text { e } f(B(\zeta ; r)) \subset B(f(\zeta) ;|f(\zeta)|)
$$

Pela proposição 4.8 existe uma função contínua $L$ satisfazendo $e^{L(w)}=w$, para todo $w \in$ $B(f(\zeta) ;|f(\zeta)|)$. Logo, dado $z \in B(\zeta ; r)$, temos

$$
e^{L(f(z))}=f(z)=e^{\varphi_{z}(1)} .
$$

Pela periodicidade da função exponencial complexa, existe $k \in \mathbb{Z}$ tal que

$$
L(f(\zeta))=\varphi_{\zeta}(1)+2 k \pi i
$$

Logo, a função $g: z \in B(\zeta ; r) \mapsto L(f(z))-2 k \pi i$ satisfaz

$$
\left\{\begin{array}{l}
e^{g(z)}=f(z) \\
g(\zeta)=\varphi_{\zeta}(1)
\end{array}\right.
$$

Consideremos $\omega \in B(\zeta ; r)$ fixado e seja $\alpha:[0,1] \rightarrow \mathbb{C}$ o segmento unindo $\zeta$ a $\omega$, dado por $\alpha(t)=\zeta+t(\omega-\zeta)$, com $t \in[0,1]$. Definamos o caminho fechado $\Gamma$ por

$$
\Gamma(t)= \begin{cases}\gamma_{\omega}(1-t), & \text { se } 0 \leq t \leq 1 \\ \gamma_{\zeta}(t-1), & \text { se } 1 \leq t \leq 2 \\ \alpha(t-2), & \text { se } 2 \leq t \leq 3\end{cases}
$$

e a função contínua

$$
\Psi(t)= \begin{cases}\varphi_{\omega}(1-t), & \text { se } 0 \leq t \leq 1 \\ \varphi_{\zeta}(t-1), & \text { se } 1 \leq t \leq 2 \\ (g \circ \alpha)(t-2), & \text { se } 2 \leq t \leq 3\end{cases}
$$

Das igualdades em 4.2 e 4.3, temos a identidade

$$
(f \circ \Gamma)(t)=e^{\Psi(t)}, \text { para todo } t \in[0,1] .
$$


Logo,

$$
\operatorname{Im}(\Psi): t \in[0,3] \mapsto \operatorname{Im}(\Psi(t))
$$

é um argumento de $f \circ \Gamma$.

Assim, temos

$$
2 \pi \operatorname{Ind}(f \circ \Gamma, 0)=\operatorname{Im}(\Psi(3))-\operatorname{Im}(\Psi(0)) .
$$

Mas, por hipótese, $\operatorname{Ind}(f \circ \Gamma, 0)=0$. Logo, $\operatorname{Im}(\Psi(0))=\operatorname{Im}(\Psi(3))$.

Ainda, como $|f \circ \Gamma(t)|=e^{\operatorname{Re}(\Psi(t))}$, para todo $t \in[0,3]$, temos que $\operatorname{Re}(\Psi)=\ln |f \circ \Gamma|$. Segue então que

$$
\begin{aligned}
g(\omega)-\Phi(\omega)= & g(\omega)-\varphi_{\omega}(1)=g(\alpha(1))-\varphi_{\omega}(1)=\Psi(3)-\Psi(0)= \\
& =\ln |(f \circ \Gamma)(3)|-\ln |(f \circ \Gamma)(0)|=0 .
\end{aligned}
$$

Portanto, para todo $z \in B(\zeta ; r)$, vale

$$
g(z)=\Phi(z)
$$

Sendo $g$ contínua em $B(\zeta ; r)$ e $\zeta$ arbitrário em $\Omega$, temos que $\Phi$ é um logaritmo contínuo de $f$ em $\Omega$, como queríamos.

$(b \Rightarrow a)$ Suponha que $g$ é um logaritmo contínuo de $f$. Logo, $\operatorname{Im}(g(z))$ é um argumento de $f$ em $\Omega$.

Seja $\gamma:[a, b] \rightarrow \Omega$ um caminho fechado. Dado que $(f \circ \gamma)(t)=|(f \circ \gamma)(t)| e^{i \operatorname{Im}(g \circ \gamma)(t)}$, para todo $t$ no domínio de $\gamma$, temos

$$
2 \pi \operatorname{Ind}(f \circ \gamma, 0)=\operatorname{Im}[(g \circ \gamma)(b)]-\operatorname{Im}[(g \circ \gamma)(a)]=\operatorname{Im}[(g \circ \gamma)(b)-(g \circ \gamma)(a)]=0
$$

Corolário 4.32. Sejam $\Omega$ um aberto simplesmente conexo de $\mathbb{C}$ e uma função $f: \Omega \rightarrow \mathbb{C}^{*}$ contínua. Então, $f$ tem um logaritmo contínuo em $\Omega$ (isto é, existe $g: \Omega \rightarrow \mathbb{C}$ contínua tal que $f(z)=e^{g(z)}$, para todo $z \in \Omega$ ).

Demonstração. Seja $\gamma$ um caminho fechado em $\Omega$. Dado que $\Omega$ é simplesmente conexo, tem-se que $\gamma$ é homotópico a um ponto de $\Omega$. Logo, o caminho $f \circ \gamma$ é homotópico a um ponto de $\mathbb{C}^{*}$. Segue que $\operatorname{Ind}(f \circ \gamma, 0)=0$ e pelo teorema anterior, $f$ tem um logaritmo contínuo em $\Omega$.

Proposição 4.33. Sejam $\Omega$ aberto conexo e uma função $f: \Omega \rightarrow \mathbb{C}^{*}$ analítica. Se g é um logaritmo contínuo de $f$ em $\Omega$, então $g$ é analítica em $\Omega$.

Demonstração. Seja $w \in \Omega$ arbitrário. Pela continuidade de $f$ podemos fixar $r>0$ tal que $B(w ; r) \subset$ $U$ e $f(B(w ; r)) \subset B(f(w) ;|f(w)|)$. Consideremos $\zeta \in \mathbb{C}$ tal que $e^{\zeta}=f(w)$. Então a função $h$, definida como segue, é um logaritmo contínuo de $f$

$$
h(z)=\zeta+\log \left(\frac{f(z)}{f(w)}\right), \text { para todo } z \in B(w ; r) .
$$


Por continuidade e conexidade, existe $k \in \mathbb{Z}$ tal que

$$
g(z)=\zeta+\log \left(\frac{f(z)}{f(w)}\right)+2 i k \pi, \text { para todo } z \in B(w ; r) .
$$

Dado que a analiticidade é de caráter local, concluímos que $g$ é analítica em $\Omega$.

O corolário a seguir será essencial nas demonstrações do Teorema da Fatoração de Weierstrass, do Pequeno Teorema de Picard e do Teorema da Aplicação de Riemann.

Corolário 4.34. Sejam $\Omega$ um aberto simplesmente conexo de $\mathbb{C}$ e uma função $f: \Omega \rightarrow \mathbb{C}^{*}$ analítica. Então,

(a) Existe $g \in \mathcal{A}(\Omega)$ tal que $f(z)=e^{g(z)}$, para todo $z \in \Omega$.

(b) Dado $n \in \mathbb{N}$, existe $h \in \mathcal{A}(\Omega)$ tal que $f(z)=[h(z)]^{n}$, para todo $z \in \Omega$.

Demonstração. (a) Pelo corolário 4.32, $f$ tem um logaritmo contínuo $g$. Segue da proposição 4.33 que $g$ é analítica.

(b) Pelo item $(a)$, existe $g$ tal que $f(z)=e^{g(z)}$, para todo $z \in \Omega$. Considerando $h$ dada por $h(z)=e^{\frac{g(z)}{n}}, \operatorname{com} z \in \Omega$, temos a função desejada.

Se $n=2, h$ como acima será denotada por $\sqrt{f}$ e é dita uma raiz quadrada de $f$.

Finalizamos esta seção com dois exemplos.

O primeiro exemplo é um resultado apresentado em [14]. Em sua prova, Narasimhan faz uso da teoria de integração.

Exemplo 4.35. Sejam $\Omega$ aberto conexo e $a, b$ pontos pertencentes a mesma componente conexa de $\mathbb{C} \backslash \Omega$. Mostraremos que existe $f \in \mathcal{A}(\Omega)$ tal que, para todo $z \in \Omega$

$$
e^{f(z)}=\frac{z-a}{z-b}
$$

Sejam $\gamma:[c, d] \rightarrow \Omega$ um caminho fechado e $g: z \in \Omega \mapsto \frac{z-a}{z-b}$. Pelo teorema 4.31 e a proposição 4.33 é suficiente mostrarmos que $\operatorname{Ind}(g \circ \gamma, 0)=0$.

Temos que

$$
(g \circ \gamma)(t)=\frac{\gamma(t)-a}{\gamma(t)-b}, \text { para todo } t \in[c, d] .
$$

Considerando $\theta_{1}$ e $\theta_{2}$ funções argumentos para os caminhos $\gamma-a$ e $\gamma-b$, obtemos que para cada $t \in[c, d]$ vale

$$
(g \circ \gamma)(t)=\frac{|\gamma(t)-a|}{|\gamma(t)-b|} \frac{e^{i \theta_{1}(t)}}{e^{i \theta_{2}(t)}}=|(g \circ \gamma)(t)| e^{i\left(\theta_{1}(t)-\theta_{2}(t)\right)}
$$

Como a e b pertencem a mesma componente conexa de $\mathbb{C} \backslash \Omega$ e, consequentemente pertencem a mesma componente conexa de $\mathbb{C} \backslash \operatorname{Imagem}(\gamma)$, temos que $\operatorname{Ind}(\gamma, a)=\operatorname{Ind}(\gamma, b)$.

Portanto,

$$
\operatorname{Ind}(g \circ \gamma, 0)=\frac{\left[\theta_{1}(d)-\theta_{2}(d)\right]-\left[\theta_{1}(c)-\theta_{2}(c)\right]}{2 \pi}=\operatorname{Ind}(\gamma, a)-\operatorname{Ind}(\gamma, b)=0 .
$$


Exemplo 4.36. Vamos determinar todos os pares de funções inteiras $f$ e $g$ que satisfaçam a relação

$$
f(z)^{2}+g(z)^{2}=1, \text { para todo } z \in \mathbb{C}
$$

Dado $z \in \mathbb{C}$, temos

$$
f(z)^{2}+g(z)^{2}=(f(z)+i g(z))(f(z)-i g(z))=1 .
$$

Logo, $f+i g$ é uma função inteira sem zeros. Como $\mathbb{C}$ é simplesmente conexo, existe uma função inteira $H$ tal que

$$
f+i g=e^{H}
$$

Mas, $f-i g=\frac{1}{f+i g}$, ent $\tilde{a} O$

$$
f-i g=e^{-H} .
$$

Somando e sutraindo, adequadamente, as igualdades em 4.4 e 4.5, obtemos

$$
\left\{\begin{array}{l}
f(z)=\frac{e^{H(z)}+e^{-H(z)}}{2}=\cos (-i H(z)), \\
g(z)=\frac{e^{H(z)}-e^{-H(z)}}{2 i}=\sin (-i H(z)) .
\end{array}\right.
$$

Portanto, $f(z)=\cos F(z)$ e $g(z)=\sin F(z)$, para alguma função inteira $F$.

É claro que se $f$ e g são dessa forma, então elas satisfazem $f(z)^{2}+g(z)^{2}=1$. 


\section{Capítulo 5}

\section{O Teorema da Aplicação de Riemann}

Neste capítulo demonstraremos o famoso Teorema da Aplicação de Riemann e daremos algumas caracterizações dos abertos simplesmente conexos do plano.

Atualmente existem três caminhos distintos para provar o Teorema da Aplicação de Riemann, os métodos utilizados nelas são: Princípio de Dirichlet, teoria potencial e o princípio extremal de Fejér-Riesz. O teorema foi afirmado primeiramente por Riemann em 1851.

A prova do Teorema da Aplicação de Riemann aqui apresentada é uma variação da apresentada por F. Riesz e L. Féjer. O mais interessante é que tal prova será obtida sem o uso de qualquer teorema de integração complexa. Nossa referências para esta seção encontram-se em [18], [23], [5] e [27].

Para definir abertos conexos conformemente equivalentes, os livros textos utilizam o conceito de holomorfia, mas nesse texto, isto não se faz necessário e nos basta a definição a seguir.

Definição 5.1. Dois abertos conexos $\Omega_{1}$ e $\Omega_{2}$ são ditos conformemente equivalentes se existe uma aplicação $\varphi: \Omega_{1} \rightarrow \Omega_{2}$ analítica e bijetora.

Pelo Teorema da Função Inversa Global (p. 41), uma função $\varphi$ como na definição acima é um isomorfismo analítico.

A seguir provaremos uma série de resultados que nos auxiliarão a demonstrar o Teorema da Aplicação de Riemann.

Teorema 5.2 (Hurwitz). Sejam $\Omega$ aberto conexo e $\left(f_{n}\right)$ uma sequência em $\mathcal{A}(\Omega)$ de funções sem zeros que converge compactamente à $f$ (em $\Omega$ ). Se $f_{n}$ não tem zeros, para todo $n \in \mathbb{N}$, então $f$ não tem zeros ou $f$ é nula.

Demonstração 1. Suponhamos por contradição que $f$ tem um zero em $a \in \Omega$ e $f$ não é constante. Pelo Princípio dos Zeros Isolados existe $r>0$ tal que $f$ não tem zeros em $S(a ; r)$.

Seja $2 m=\min _{S(a ; r)}|f(z)|$. Como $m>0$, existe $N \in \mathbb{N}$ tal que $\left\|f_{N}-f\right\|_{S(a ; r)}<m$. Pelo Princípio do Módulo Máximo (p. 37), temos que

$$
\left|f_{N}(a)\right|=\left|f_{N}(a)-f(a)\right|<m \text {. }
$$

Por outro lado, dado $z \in S(a ; r)$, vale

$$
\left|f_{N}(z)\right| \geq|f(z)|-\left|f_{N}(z)-f(z)\right|>2 m-m=m .
$$


Logo, $\left|f_{N}\right|$ tem ponto de mínimo interior a $B(a ; r)$ e portanto $f_{N}$ se anula em $\Omega$.

Como todo aberto conexo é conexo por caminhos e reciprocamente, todo aberto conexo por caminhos é conexo (vide Teorema 4.10), obtemos a proposição 5.3 e esta será útil para obtermos um corolário do teorema 5.2 .

Proposição 5.3. Seja $\Omega$ um aberto conexo de $\mathbb{C}$. Se $a \in \Omega$, então $\Omega \backslash\{a\}$ é conexo.

O corolário a seguir será utilizado na prova do teorema 5.9.

Corolário 5.4. Sejam $\Omega$ um aberto conexo e $\left(f_{n}\right)$ uma sequência em $\mathcal{A}(\Omega)$ de funções injetoras convergindo compactamente a $f$ (em $\Omega$ ). Então $f$ é injetora ou $f$ é constante.

Demonstração. Suponhamos que $f$ não é constante e consideremos $a$ e $b$ distintos em $\Omega$. Temos que $\left(f_{n}-f_{n}(a)\right)$ converge compactamente em $\Omega \backslash\{a\}$ à função $f-f(a)$ e $\left(f_{n}-f_{n}(a)\right)$ é uma sequência de funções sem zeros em $\Omega \backslash\{a\}$. Pela proposição 5.3, o conjunto $\Omega \backslash\{a\}$ é conexo. Logo, pelo teorema 5.2, ou $f-f(a) \equiv 0$, ou $f-f(a)$ não se anula em $\Omega \backslash\{a\}$. Sendo $f$ não constante, segue que $f(a) \neq f(b)$ e, portanto, $f$ é injetora.

Para simplificar, usaremos a notação $\mathbb{D}=B(0 ; 1)$.

Dado $a \in \mathbb{D}$, definimos

$$
\varphi_{a}: z \in \mathbb{D} \mapsto \frac{z-a}{1-\bar{a} z} \in \mathbb{C} .
$$

Dada $f \in \mathcal{A}(\mathbb{D})$, dizemos que $f$ é um automorfismo analítico de $\mathbb{D}$ se $f: \mathbb{D} \rightarrow \mathbb{D}$ é bijetora, com $f$ e $f^{-1}$ holomorfas.

Lema 5.5. Seja $f \in \mathcal{A}(\mathbb{D})$. Então $f$ é um automorfismo analítico de $\mathbb{D}$ se e somente se existem $\omega \in S^{1}$ e $a \in \mathbb{D}$ tais que

$$
f(z)=\omega \varphi_{a}(z)
$$

onde

$$
\varphi_{a}(z)=\frac{z-a}{1-\bar{a} z}, \text { para todo } z \in \mathbb{D} .
$$

Demonstração. $(\Leftarrow)$ A função $\varphi_{a}$ é não constante e analítica no aberto $B\left(0 ;|a|^{-1}\right)$ contendo $\mathbb{D}$. Além disso, se $|z|=1(z \bar{z}=1)$, temos

$$
\left|\varphi_{a}(z)\right|=\left|\frac{z-a}{1-\bar{a} z}\right|=\left|\frac{z-a}{z \bar{z}-\bar{a} z}\right|=1 .
$$

Logo, $\varphi_{a}\left(S^{1}\right) \subset S^{1}$ e pelo Princípio do Módulo Máximo (p. 37) $\varphi_{a}(\mathbb{D}) \subset \mathbb{D}$. Analogamente,

$$
\varphi_{-a}\left(S^{1}\right) \subset S^{1} \text { e } \varphi_{-a}(\mathbb{D}) \subset \mathbb{D} .
$$

Vale também, para todo $z \in \mathbb{D}$

$$
\left(\varphi_{a} \circ \varphi_{-a}\right)(z)=z \text { e }\left(\varphi_{-a} \circ \varphi_{a}\right)(z)=z
$$


Concluímos então que $\varphi_{a}$ é um automorfismo analítico de $\mathbb{D}$ e $\left(\varphi_{a}\right)^{-1}=\varphi_{-a}$. Ainda, como $\omega \in S^{1}$, segue que $\omega \varphi_{a}$ é um automorfismo de $\mathbb{D}$ (com inversa dada por $h(z)=\varphi_{-a}\left(z \omega^{-1}\right)$ ).

$(\Rightarrow)$ Sejam $b=f(0)$ e $F=\varphi_{b} \circ f$.

A função $F$ é um automorfismo da bola $\mathbb{D}$ e $F(0)=\varphi_{b}(b)=0$. Pelo Lema de Schwarz (p. 45), $|F(z)| \leq|z|$, para todo $z \in \mathbb{D}$. A função $F^{-1}$ também satisfaz as condições do Lema de Schwarz. Logo, $\left|F^{-1}(z)\right| \leq|z|$ para todo $z \in \mathbb{D}$.

Consequentemente, para cada $z \in \mathbb{D}$

$$
|z|=\left|F^{-1}(F(z))\right| \leq|F(z)| \leq|z|
$$

Aplicando novamente o Lema de Schwarz, temos que existe $\omega \in S^{1}$ tal que $F(z)=\omega z$ em $\mathbb{D}$.

Finalmente, lembrando que $\omega \bar{\omega}=1$, obtemos

$$
f(z)=\left(\varphi_{-b} \circ F\right)(z)=\varphi_{-b}(\omega z)=\omega \frac{z+b \bar{\omega}}{1+\overline{b \bar{\omega}} z}=\omega \varphi_{-b \bar{\omega}}(z)
$$

Para simplificarmos os enunciados seguintes, damos a definição abaixo.

Definição 5.6. Seja $\Omega$ aberto conexo tal que $0 \in \Omega$. Se toda $f \in \mathcal{A}(\Omega)$ que não se anula tem uma raiz quadrada, $\Omega$ é chamado de domínio para raiz quadrada.

Na demonstração do Lema de Köebe usaremos o lema anterior, 5.5 e o Lema de Schwarz (p. 45).

A função $\kappa$ do lema abaixo é chamada de expansão de $\Omega$ a $\mathbb{D}$ relativa a origem.

Lema 5.7 (Köebe). Seja $\Omega \subset \mathbb{D}$ um domínio para raiz quadrada tal que $\Omega \neq \mathbb{D}$. Então existe uma aplicação $\kappa \in \mathcal{A}(\Omega)$ com as seguintes propriedades

(a) $\kappa(0)=0$ e $\kappa(\Omega) \subset \mathbb{D}$;

(b) $\kappa: \Omega \rightarrow \mathbb{D}$ é injetora;

(c) $|\kappa(z)|>|z|$, para todo $z \in \Omega \backslash\{0\}$.

Demonstração. Seja $a \in \mathbb{D} \backslash \Omega$ fixado e consideremos a aplicação $\varphi_{a}$ com domínio em $\mathbb{D}$.

A função $\varphi:=\left.\varphi_{a}\right|_{\Omega}: \Omega \rightarrow \varphi_{a}(\Omega)$ é analítica, bijetora e não possui zeros em $\Omega$. Por hipótese, existe $g \in \mathcal{A}(\Omega)$ tal que $g(z)^{2}=\varphi(z)$, para cada $z \in \Omega$.

Observemos que $g(\Omega) \subset \mathbb{D}$, pois dado $z \in \Omega$ temos que

$$
|g(z)|^{2}=|\varphi(z)|<1
$$

e mais, $g: \Omega \rightarrow g(\Omega)$ é bijetora (se $g(z)=g(w)$, então $\varphi(z)=\varphi(w)$ ).

Consideremos o ponto $b=g(0) \in \mathbb{D}$, a função $\varphi_{b}$ com domínio em $\mathbb{D}$ e as aplicações bijetoras

$$
\begin{gathered}
\psi:=\left.\varphi_{b}\right|_{g(\Omega)}: g(\Omega) \rightarrow \varphi_{b}(g(\Omega)), \\
\kappa:=\psi \circ g: \Omega \rightarrow \psi(g(\Omega)) .
\end{gathered}
$$

A bijeção $\kappa$ definida no aberto conexo $\Omega$ é analítica e injetora. Logo, pelo Teorema da Função Inversa Global (p. 41), $\kappa: \Omega \rightarrow \kappa(\Omega)$ é um isomorfismo analítico (com inversa analítica) satisfazendo $\kappa(0)=0$, ainda, como $\kappa(\Omega)=\psi(g(\Omega))$, temos que $\kappa(\Omega) \subset \mathbb{D}$. 
Em resumo, pelo Teorema da Aplicação Aberta (p. 39) e o Teorema da Função Inversa Global, são isomorfismos analíticos entre abertos as seguintes funções

$$
\left\{\begin{array}{l}
\varphi: \Omega \rightarrow \varphi(\Omega), \text { onde } \varphi(\Omega)=\varphi_{a}(\Omega) \\
g: \Omega \rightarrow g(\Omega), \text { e } g(z)^{2}=\varphi(z), \text { para todo } z \in \Omega \\
\psi: g(\Omega) \rightarrow \psi(g(\Omega)), \text { onde } \psi(g(\Omega))=\varphi_{b}(g(\Omega)) \\
\kappa: \Omega \rightarrow \kappa(\Omega), \text { onde } \kappa(\Omega)=\psi(g(\Omega))
\end{array}\right.
$$

e também

$$
\left\{\begin{array}{l}
\varphi^{-1}: \varphi(\Omega) \rightarrow \Omega, \text { e } \varphi^{-1}(\zeta)=\varphi_{-a}(\zeta), \text { para todo } \zeta \in \varphi(\Omega) \\
g^{-1}: g(\Omega) \rightarrow \Omega, \\
\psi^{-1}: \psi(g(\Omega)) \rightarrow g(\Omega), \text { e } \psi^{-1}(\zeta)=\varphi_{-b}(\zeta), \text { para todo } \zeta \in \psi(g(\Omega)) \\
\kappa^{-1}: \kappa(\Omega) \rightarrow \Omega, \text { e } \kappa^{-1}=g^{-1} \circ \psi^{-1}
\end{array}\right.
$$

A seguir mostraremos a fatoração

$$
\kappa^{-1}(\zeta)=\left(\varphi_{-a} \circ h \circ \varphi_{-b}\right)(\zeta), \text { para todo } \zeta \in \kappa(\Omega), \text { onde } h: z \in \mathbb{D} \mapsto z^{2} \in \mathbb{D} \text {. }
$$

Consideremos a função bijetora

$$
r:=g \circ \varphi^{-1}: \varphi(\Omega) \rightarrow g(\Omega)
$$

Como $g(z)^{2}=\varphi(z)$, para todo $z \in \Omega$, obtemos

$$
r(\zeta)^{2}=g\left[\varphi^{-1}(\zeta)\right]^{2}=\varphi\left[\varphi^{-1}(\zeta)\right]=\zeta, \text { para todo } \zeta \in \varphi(\Omega)
$$

Logo

$$
z^{2}=\left[r\left(r^{-1}(z)\right)\right]^{2}=r^{-1}(z), \text { para todo } z \in g(\Omega) .
$$

Dado que $r=g \circ \varphi^{-1}$, segue que $g^{-1}=\varphi^{-1} \circ r^{-1}$ e por 5.1 , se $z \in g(\Omega)$, então

$$
g^{-1}(z)=\varphi^{-1}\left(r^{-1}(z)\right)=\varphi_{-a}\left(r^{-1}(z)\right)=\varphi_{-a}\left(z^{2}\right)=\frac{z^{2}+a}{1+\bar{a} z^{2}} .
$$

Pelas igualdades em 5.1 decorre que para todo $\zeta \in \kappa(\Omega)=\psi(g(\Omega))$ temos

$$
\kappa^{-1}(\zeta)=g^{-1}\left(\psi^{-1}(\zeta)\right)=\frac{\varphi_{-b}(\zeta)^{2}+a}{1+\bar{a} \varphi_{-b}(\zeta)^{2}}=\left(\varphi_{-a} \circ h \circ \varphi_{-b}\right)(\zeta)
$$

Isto completa a fatoração de $k^{-1}$.

Notemos que $b^{2}=-a$, pois $g(0)=b$ e $g(0)^{2}=\varphi(0)=\varphi_{a}(0)=-a$. Logo, a extensão de $k^{-1}$ definida por $H:=\varphi_{-a} \circ h \circ \varphi_{-b}: \mathbb{D} \rightarrow \mathbb{D}$ não é uma rotação. Caso contrário, existe $\omega \in S^{1}$ tal que

$$
\left\{\begin{array}{l}
H(-b)=-\omega b \\
H(-b)=\varphi_{-a}\left[\varphi_{-b}(-b)^{2}\right]=\varphi_{-a}(0)=a
\end{array}\right.
$$

o que implica

$$
-\omega b=a=-b^{2}
$$


e portanto, $\omega=b$, mas $b \in \mathbb{D}(g(\Omega) \subset \mathbb{D})$, o que é uma contradição.

Ainda, como $0 \in \kappa(\Omega) \subset \mathbb{D}$ e $\kappa^{-1}$ é uma restrição de $H$, temos que $H(0)=\kappa^{-1}(0)=0$. Logo, aplicando o Lema de Schwarz (p. 45) à função $H$ obtemos

$$
|H(z)|<|z| \text {, para todo } z \in \mathbb{D} \backslash\{0\} \text {. }
$$

Consequentemente obtemos que $\left|\kappa^{-1}(\zeta)\right|<|\zeta|$, se $\zeta \in \kappa(\Omega) \backslash\{0\}$ e, finalmente, segue que dado $z \in \Omega \backslash\{0\}$ vale

$$
|z|=\left|\kappa^{-1}(\kappa(z))\right|<|\kappa(z)|
$$

Lema 5.8. Se $\varphi: \Omega_{1} \rightarrow \Omega_{2}$ é isomorfismo analítico e toda $f \in \mathcal{A}\left(\Omega_{1}\right)$ que não se anula tem uma raiz quadrada, então toda $h \in \mathcal{A}\left(\Omega_{2}\right)$ que não se anula tem uma raiz quadrada.

Demonstração. Dada $h \in \mathcal{A}\left(\Omega_{2}\right)$ e sem zeros em $\Omega_{2}$, basta considerarmos $F \in \mathcal{A}\left(\Omega_{1}\right)$ tal que $F^{2}=h \circ \varphi$, pois é trivial verificarmos que

$$
\left(F \circ \varphi^{-1}\right)^{2}=h
$$

O teorema a seguir será a principal ferramenta para a demonstração do Teorema da Aplicação de Riemann. Provaremos que dado um aberto conexo distinto de $\mathbb{C}$, com a propriedade de que toda $f \in \mathcal{A}(\Omega)$ que não se anula tem uma raiz quadrada, então existe um isomorfismo analítico entre ele e um domínio para raiz quadrada, assim como um isomorfismo entre o domínio para raiz quadrada e a bola $\mathbb{D}$. Não é difícil ver que o Teorema da Aplicação de Riemann decorrerá disso, pois já provamos o corolário 4.34 (toda função analítica e sem zeros num aberto simplesmente conexo tem raiz $n$-ésima).

Teorema 5.9. Seja $\Omega$ um aberto conexo não vazio tal que $\Omega \neq \mathbb{C}$. Se toda $f \in \mathcal{A}(\Omega)$ sem zeros em $\Omega$ tem uma raiz quadrada, então existe um isomorfismo analítico entre $\Omega$ e $\mathbb{D}$.

Demonstração. Dividiremos a prova em duas partes.

Afirmação 1. Existe um isomorfismo analítico $h_{0}$ entre $\Omega$ e um domínio para raiz quadrada $\Omega_{0} \subset \mathbb{D}$.

Seja $a \in \mathbb{C} \backslash \Omega$. Por hipótese, existe $g \in \mathcal{A}(\Omega)$ tal que

$$
g(z)^{2}=z-a, \text { se } z \in \Omega .
$$

A função $g$ é claramente injetora. Ainda, se $w \in g(\Omega)$, então $-w \notin g(\Omega)$. De fato, se $z_{1}, z_{2} \in \Omega$ são tais que $g\left(z_{1}\right)=w=-g\left(z_{2}\right)$, então temos

$$
g\left(z_{1}\right)^{2}=g\left(z_{2}\right)^{2}
$$

e portanto, $z_{1}=z_{2}$ e $w=-w$. Logo, $w=0$ e obtemos uma contradição, pois $0 \notin g(\Omega)$.

Fixemos $w_{0} \in g(\Omega)$. Pelo Teorema da Aplicação Aberta (p. 39) existe $r>0$ tal que $B\left(w_{0} ; r\right) \subset$ $g(\Omega)$. 
Logo,

$$
g(\Omega) \cap B\left(-w_{0} ; r\right)=\emptyset .
$$

Com efeito, se $w \in B\left(-w_{0} ; r\right)$, então $-w \in B\left(w_{0} ; r\right) \subset g(\Omega)$ e, pelo que já vimos, $w \notin g(\Omega)$.

Devido a intersecção em destaque, temos que

$$
\left|g(z)+w_{0}\right| \geq r, \text { para todo } z \in \Omega \text {. }
$$

Pelos comentários anteriores, a função

$$
h: z \in \Omega \mapsto \frac{1}{g(z)+w_{0}}
$$

é analítica, injetora e satisfaz

$$
|h(z)| \leq \frac{1}{r}, \text { para todo } z \in \Omega
$$

Consideremos $z_{0} \in \Omega$ e $0<\varepsilon<\frac{r}{2}$ fixados e definamos a função injetora

$$
h_{0}: z \in \Omega \mapsto \varepsilon\left(h(z)-h\left(z_{0}\right)\right) .
$$

Pelo Teorema da Aplicação Aberta (p. 39), $\Omega_{0}=h_{0}(\Omega)$ é um aberto conexo e $0 \in \Omega_{0} \subset \mathbb{D}$. Pois,

$$
h_{0}\left(z_{0}\right)=0 \quad \text { e }\left|h_{0}(z)\right| \leq \varepsilon\left(|h(z)|+\left|h\left(z_{0}\right)\right|\right)<\frac{r}{2} \cdot \frac{1}{r}+\frac{r}{2} \cdot \frac{1}{r}=1 .
$$

Aplicando o Teorema da Função Inversa Global (p. 41), segue que $h_{0}: \Omega \rightarrow \Omega_{0}$ é um isomorfismo analítico e pelo lema 5.8, o conjunto $\Omega_{0}$ é um domínio para raiz quadrada.

Afirmação 2. Seja $\mathcal{F}=\left\{f \in \mathcal{A}\left(\Omega_{0}\right): f\left(\Omega_{0}\right) \subset \mathbb{D}\right.$, com $f$ injetora e $\left.f(0)=0\right\}$. Então existe $F \in \mathcal{F}$ tal que $F: \Omega_{0} \rightarrow \mathbb{D}$ é um isomorfismo analítico.

Inicialmente notemos que a família $\mathcal{F}$ é não vazia, pois contém a função $h: z \in \Omega_{0} \mapsto z$.

Fixemos um ponto arbitrário $z_{0} \in \Omega_{0}$ não nulo. Temos que $f\left(z_{0}\right) \neq 0$, para toda $f \in \mathcal{F}$ e, portanto

$$
0<\alpha=\sup _{f \in \mathcal{F}}\left|f\left(z_{0}\right)\right| \leq 1 .
$$

Mostraremos a seguir que existe $F \in \mathcal{F}$ tal que $\left|F\left(z_{0}\right)\right|=\alpha$, com $F: \Omega_{0} \rightarrow \mathbb{D}$ um isomorfismo analítico.

Dado que

$$
\alpha \in \overline{\left\{\left|f\left(z_{0}\right)\right|: f \in \mathcal{F}\right\}},
$$

existe uma sequência $\left(f_{n}\right)$ de funções em $\mathcal{F}$ tal que $\left|f_{n}\left(z_{0}\right)\right| \rightarrow \alpha$.

A família $\mathcal{F}$ é localmente limitada. Segue do Teorema de Montel (p. 50) que existe uma subsequência $\left(f_{n_{k}}\right)$ de $\left(f_{n}\right)$ tal que $\left(f_{n_{k}}\right)$ converge compactamente em $\Omega_{0}$ à uma função $F \in \mathcal{A}\left(\Omega_{0}\right)$.

Como $\left(f_{n_{k}}\right)$ converge simplesmente à $F$, obtemos que

$$
F(0)=\lim _{k \rightarrow \infty} f_{n_{k}}(0)=0 \text { e }\left|F\left(z_{0}\right)\right|=\lim _{k \rightarrow \infty}\left|f_{n_{k}}\left(z_{0}\right)\right|=\alpha .
$$

Logo, a função $F$ é não constante e, pelo corolário 5.4 (limite de funções injetoras), $F$ é injetora. Segue do Teorema da Função Inversa Global (p. 41) que $F: \Omega_{0} \rightarrow F\left(\Omega_{0}\right)$ é um isomorfismo 
analítico.

É trivial verificarmos que $F\left(\Omega_{0}\right) \subset \mathbb{D}$. Resta mostrarmos que $F\left(\Omega_{0}\right)=\mathbb{D}$.

Pelo lema 5.8, o conjunto $F\left(\Omega_{0}\right)$ é um domínio para raiz quadrada. Suponhamos que $F\left(\Omega_{0}\right) \neq$ $\mathbb{D}$. Então tal conjunto satisfaz as condições do Lema de Köebe e portanto, existe uma função $\kappa: F\left(\Omega_{0}\right) \rightarrow \mathbb{D}$ analítica tal que

$$
\kappa(0)=0, \kappa \text { é injetora e }|\kappa(z)|>|z| \text {, para todo } z \in F\left(\Omega_{0}\right) \backslash\{0\} .
$$

Pelos comentários anteriores temos que $\kappa \circ F \in \mathcal{F}$ e, no entanto

$$
\left|(\kappa \circ F)\left(z_{0}\right)\right|>\left|F\left(z_{0}\right)\right|=\alpha=\sup _{f \in \mathcal{F}}\left|f\left(z_{0}\right)\right|,
$$

o que é uma contradição. Portanto, $F\left(\Omega_{0}\right)=\mathbb{D}$.

Concluímos pelas afirmações (1) e (2) que as seguintes funções são analíticas, suas respectivas inversas também são analíticas e, portanto são isomorfismos analíticos

$$
\begin{gathered}
h_{0}: \Omega \rightarrow \Omega_{0}, \\
F: \Omega_{0} \rightarrow \mathbb{D}, \\
F \circ h_{0}: \Omega \rightarrow \mathbb{D} .
\end{gathered}
$$

Provaremos abaixo um dos teoremas centrais da Análise Complexa, o Teorema da Aplicação de Riemann. É claro que se um aberto de $\mathbb{C}$ for homeomorfo a $\mathbb{D}$, então ele é simplesmente conexo. Com o Teorema da Aplicação de Riemann veremos que vale a recíproca.

A hipótese abaixo de que $\Omega \neq \mathbb{C}$ foi feita, pois qualquer função analítica $f: \mathbb{C} \rightarrow \mathbb{D}$ é limitada e pelo Teorema de Liouville é constante.

Teorema 5.10 (Teorema da Aplicação de Riemann). Se $\Omega$ é um aberto simplesmente conexo não vazio e tal que $\Omega \neq \mathbb{C}$, então $\Omega$ é conformemente equivalente a $\mathbb{D}$.

Demonstração. Dado que $\Omega$ é simplesmente conexo, decorre do corolário 4.34 (existência de raiz $n$-ésima - p. 69) que toda função $f \in \mathcal{A}(\Omega)$ que não se anula possui uma raiz quadrada.

Por hipótese, $\Omega \neq \mathbb{C}$. Logo, pelo teorema 5.9 existe uma bijeção analítica entre os abertos conexos $\Omega$ e $\mathbb{D}$. Assim, por definição, $\Omega$ e $\mathbb{D}$ são conformemente equivalentes.

É trivial verificarmos o resultado a seguir.

Corolário 5.11. Se $\Omega_{1}$ e $\Omega_{2}$ são abertos simplesmente conexos distintos de $\mathbb{C}$, então eles são conformemente equivalentes.

Como consequência do Teorema da Aplicação de Riemann e dos resultados obtidos na seção 4.4, temos o seguinte teorema que nos dá um caracterização dos abertos simplesmente conexos do plano. 
Teorema 5.12. Seja $\Omega$ um aberto conexo não vazio que não é o plano todo. As seguintes afirmações são equivalentes.

(a) $\Omega$ é homeomorfo à bola aberta $\mathbb{D}$;

(b) $\Omega$ é simplesmente conexo;

(c) $\operatorname{Ind}(f \circ \gamma, 0)=0$, para todo $\gamma$ caminho fechado em $\Omega$ e $f \in \mathcal{C}(\Omega)$ sem zeros;

(d) Se $f \in \mathcal{C}(\Omega)$ não se anula, então existe $g \in \mathcal{C}(\Omega)$ tal que $f=\exp (g)$;

(e) Se $f \in \mathcal{A}(\Omega)$ não se anula, então existe $g \in \mathcal{A}(\Omega)$ tal que $f=\exp (g)$;

( $f)$ Se $f \in \mathcal{A}(\Omega)$ não se anula, então $f$ tem uma raiz quadrada;

(g) $\Omega$ é conformemente equivalente a $\mathbb{D}$.

Demonstração. $(a) \Rightarrow(b)$ Sejam $\gamma:[0,1] \rightarrow \Omega$ caminho fechado e $\Phi: \Omega \rightarrow \mathbb{D}$ homeomorfismo. Logo, a função $H:[0,1] \times[0,1] \rightarrow \Omega$ dada por

$$
H(x, t)=\Phi^{-1}[t \Phi(\gamma(x))], \text { para todo }(x, t) \in[0,1] \times[0,1],
$$

é uma $\Omega$-homotopia entre $\gamma$ e $\Phi^{-1}(0)$.

$(b) \Rightarrow(c)$ Trivial.

$(c) \Rightarrow(d)$ Consequência do teorema 4.31 (existência de logaritmo contínuo - p. 66).

$(d) \Rightarrow(e)$ Consequência da proposição 4.33 (o logaritmo de função analítica é uma função analítica - p. 68).

$(e) \Rightarrow(f)$ Decorre do corolário 4.34 (existência de raiz n-ésima - p. 69).

$(f) \Rightarrow(g)$ Decorre do teorema 5.9 (p. 78).

$(g) \Rightarrow(a)$ Trivial. 


\section{Apêndice A}

\section{Apêndice}

Apresentaremos a seguir provas do Teorema da Fatoração de Weierstrass e do Pequeno Teorema de Picard, ambos para funções inteiras no sentido de Weierstrass.

\section{A.1 O Teorema da Fatoração de Weierstrass}

Iniciamos esta seção com a definição de produto infinito e algumas de suas propriedades e finalizamos com a prova do Teorema da Fatoração de Weierstrass. Nossas referências para esta seção são [3], [1] e [21].

Definição A.1. Seja $\left(z_{n}\right)_{n \geq 1}$ uma sequência de números complexos. Então $\lim _{n \rightarrow \infty} \prod_{k=1}^{n} z_{k}$ é dito o produto infinito dos números $z_{n}$ e denotado por

$$
\prod_{k=1}^{\infty} z_{n}
$$

Se $\lim _{n \rightarrow \infty} \prod_{k=1}^{n} z_{k}$ existe e é diferente de zero, dizemos que o produto infinito $\prod_{n=1}^{\infty} z_{n}$ converge.

Se $\lim _{n \rightarrow \infty} \prod_{k=1}^{n} z_{k}=0$, dizemos que o produto infinito diverge a zero.

A sequência $\left(p_{n}\right)$ dada por $p_{n}=\prod_{k=1}^{n} z_{k}$, onde $n \geq 1$, é dita a sequência dos produtos parciais de $\prod_{n=1}^{\infty} z_{n}$

Considere $\left(z_{n}\right)_{n \geq 1}$ uma sequência de números reais estritamente positivos. Então, para cada $n \in \mathbb{N}$, existe $x_{n} \in \mathbb{R}$ tal que $z_{n}=\exp \left(x_{n}\right)$. Neste caso, temos a igualdade

$$
\prod_{n=1}^{\infty} z_{n}=\exp \left[\sum_{n=1}^{\infty} x_{n}\right]
$$

Logo, a convergência (divergência) de um dos membros da igualdade em A.1 implica a convergência (divergência) do outro membro. 
Seja $\prod_{n=1}^{\infty} z_{n}$ um produto infinito convergente e $\left(p_{n}\right)$ sua sequência dos produtos parciais. Então temos

$$
\lim _{n \rightarrow \infty} z_{n}=\lim _{n \rightarrow \infty} \frac{p_{n}}{p_{n-1}}=1
$$

Proposição A.2. Seja $\left(z_{n}\right)$ uma sequência de números complexos não nulos. O produto $\prod_{n=1}^{\infty} z_{n}$ converge se e somente se para todo $\varepsilon>0$, existe $n_{0} \in \mathbb{N}$ tal que

$$
\left|z_{n+1} z_{n+2} \cdots z_{n+k}-1\right|<\varepsilon \text {, se } n>n_{0} \text {, para todo } k \in \mathbb{N} \text {. }
$$

Demonstração. Sejam $p_{n}=z_{1} z_{2} \cdots z_{n}, \operatorname{com} n \in \mathbb{N}$ e $p=\lim _{n \rightarrow \infty} p_{n}$.

Suponhamos que o produto $\prod_{n=1}^{\infty} z_{n}$ converge. Como $p \neq 0$, existe $k_{0} \in \mathbb{N}$ tal que $\left|p_{n}\right|>\frac{|p|}{2}$, se $n>k_{0}$ e, portanto, existe $M>0$ tal que $\left|p_{n}\right|>M$, para todo $n \in \mathbb{N}$.

Pelo Critério de Cauchy para sequências numéricas, dado $\varepsilon>0$, existe $n_{0} \in \mathbb{N}$ tal que

$$
\left|p_{n+k}-p_{n}\right|<\varepsilon M, \text { se } n \geq n_{0} \text { e } k \in \mathbb{N} \text {. }
$$

Logo, dividindo a expressão acima por $\left|p_{n}\right|$ temos

$$
\left|z_{n+1} z_{n+2} \cdots z_{n+k}-1\right|<\varepsilon, \text { se } n>n_{0}, \text { para todo } k \in \mathbb{N}
$$

Provaremos a recíproca. Seja $\varepsilon>0$. Sem perda de generalidade podemos supor que $\varepsilon<\frac{1}{2}$. Por hipótese, existe $m_{0} \in \mathbb{N}$ tal que

$$
\left|z_{n+1} z_{n+2} \cdots z_{n+k}-1\right|<\varepsilon \text {, se } n>m_{0} \text {, para todo } k \in \mathbb{N} \text {. }
$$

Seja $q_{n}=z_{m_{0}+1} z_{m_{0}+2 \ldots} \ldots z_{n}$, onde $n>m_{0}$. Segue então

$$
\frac{1}{2} \leq\left|q_{n}\right| \leq \frac{3}{2}, \text { se } n>m_{0}
$$

Logo, se $\left(q_{n}\right)$ converge, seu limite é não nulo.

Mostremos que $\left(q_{n}\right)$ converge. Para $n>m_{0}$ e $k \in \mathbb{N}$ temos

$$
\left|\frac{q_{n+k}}{q_{n}}-1\right|=\left|z_{n+1} z_{n+2} \cdots z_{n+k}-1\right|
$$

donde segue

$$
\left|q_{n+k}-q_{k}\right|<\left|q_{n}\right| \varepsilon<\frac{3}{2} \varepsilon
$$

Portanto, pelo Critério de Cauchy, a sequência $\left(q_{n}\right)$ converge a um número complexo não nulo e, consequentemente

$$
\prod_{n=1}^{\infty} z_{n} \text { existe e é não nulo. }
$$


Proposição A.3. Seja $\left(a_{n}\right)$ sequência de números reais satisfazendo $a_{n} \geq 0$, para todo $n \in \mathbb{N}$. Então $\prod_{n=1}^{\infty}\left(1+a_{n}\right)$ converge se e somente se $\sum_{n=1}^{\infty} a_{n}$ converge.

Demonstração. Sejam $s_{n}=a_{1}+a_{2}+\cdots+a_{n}$ e $p_{n}=\left(1+a_{1}\right)\left(1+a_{2}\right) \cdots\left(1+a_{n}\right)$, para todo $n \in \mathbb{N}$. É claro que $\left(s_{n}\right)$ e $\left(p_{n}\right)$ são sequências crescentes e, portanto, é suficiente mostrarmos que $\left(s_{n}\right)$ é limitada se e somente se $\left(p_{n}\right)$ é limitada.

Como $1+x \leq e^{x}$, para todo $x \geq 0$, dado $n \in \mathbb{N}$, temos que

$$
s_{n} \leq p_{n}=\left(1+a_{1}\right)\left(1+a_{2}\right) \cdots\left(1+a_{n}\right) \leq e^{a_{1}+a_{2}+\cdots a_{n}}=e^{s_{n}} .
$$

Convém notarmos que se $\left(p_{n}\right)$ converge, seu limite é não nulo.

Proposição A.4. Seja $\left(z_{n}\right)$ uma sequência de números complexos tal que $z_{n} \neq-1$, para todo $n \in \mathbb{N}$. A convergência do produto $\prod_{n=1}^{\infty}\left(1+\left|z_{n}\right|\right)$ implica a convergência de $\prod_{n=1}^{\infty}\left(1+z_{n}\right)$.

Demonstração. Decorre da proposição A.2 e da seguinte desigualdade

$$
\begin{gathered}
\left|\left(1+z_{n+1}\right)\left(1+z_{n+2}\right) \cdots\left(1+z_{n+k}\right)-1\right|= \\
\left|1+\sum_{p=2}^{k}\left[\sum_{\nu_{1}<\nu_{2}<\cdots<\nu_{p}} z_{\nu_{1}} z_{\nu_{2}} \cdots z_{\nu_{p}}\right]+\sum_{m=1}^{k} z_{n+m}-1\right| \leq \\
\leq 1+\sum_{p=2}^{k}\left[\sum_{\nu_{1}<\nu_{2}<\cdots<\nu_{p}}\left|z_{\nu_{1}}\right|\left|z_{\nu_{2}}\right| \cdots\left|z_{\nu_{p}}\right|\right]+\sum_{m=1}^{k}\left|z_{n+m}\right|-1= \\
=\left|\left(1+\left|z_{n+1}\right|\right)\left(1+\left|z_{n+2}\right|\right) \cdots\left(1+\left|z_{n+k}\right|\right)-1\right| .
\end{gathered}
$$

Comentário A.5. É possível provar (vide Apêndice B) que se $\left(z_{n}\right)$ é uma sequência de números complexos tal que $\operatorname{Re}\left(z_{n}\right)>0$, para todo $n \in \mathbb{N}$, então a série $\sum_{n=1}^{\infty} \log \left(z_{n}\right)$ converge se e somente se $\prod_{n=1}^{\infty} z_{n}$ converge. Neste caso, se

$$
\sum_{n=1}^{\infty} \log \left(z_{n}\right)=s \text { e } \prod_{n=1}^{\infty} z_{n}=p
$$

vale que

$$
p=\exp (s)
$$

Dada uma sequência $\left(f_{n}\right)$ de funções definidas num subconjunto $\Omega$ de $\mathbb{C}$, escrevemos

$$
f(z)=\prod_{n=1}^{\infty} f_{n}(z)
$$


para dizer que $f(z)=\lim _{n \rightarrow \infty} \prod_{k=1}^{n} f_{n}(z)$. Dizemos que $\prod_{n=1}^{\infty} f_{n}(z)$ converge uniformemente em $\Omega$ se existe $n_{0} \in \mathbb{N}$ tal que a sequência

$$
\left(\prod_{k=n_{0}+1}^{n} f_{k}\right)_{n \geq n_{0}}
$$

converge uniformemente à uma função $f$ sem zeros em $\Omega$. Por fim, o produto $f(z)=\prod_{n=1}^{\infty} f_{n}(z)$ converge compactamente em $\Omega$, se converge uniformemente sobre os compactos de $\Omega$.

O seguinte lema é uma versão do Teste $\mathrm{M}$ de Weierstrass para produtos infinitos.

Relembremos que dada $f$ contínua num aberto $\Omega$ e $K \subset \Omega$ compacto, denotamos

$$
\|f\|_{K}=\max \{|f(z)|: z \in K\}
$$

Lema A.6. Sejam $K \subset \mathbb{C}$ compacto e $\left(f_{n}\right)$ uma sequência de funções contínuas definidas em $K$ com valores em $\mathbb{C}$. Se $\left\|f_{n}\right\|_{K}=M_{n}$, para cada $n \in \mathbb{N} e \sum_{n=1}^{\infty} M_{n}<\infty$, então a função

$$
f(z)=\prod_{n=1}^{\infty}\left(1+f_{n}(z)\right)
$$

converge uniformemente em $K$. Ainda, $f\left(z_{0}\right)=0$, para algum $z_{0} \in K$, se e somente se $f_{m}\left(z_{0}\right)=-1$, para algum $m \in \mathbb{N}$.

Demonstração. Seja $\varepsilon>0$, sem perda de generalidade podemos supor que $\varepsilon<\frac{1}{2}$. Pelo Critério de Cauchy (para sequência de funções), existe $n_{0} \in \mathbb{N}$ tal que

$$
\left|f_{n+1}(z)\right|+\cdots+\left|f_{n+p}(z)\right|<\varepsilon, \text { para todos } n>n_{0}, z \in K \text { e } p \in \mathbb{N} .
$$

Como $\left\|f_{n}\right\|_{K}<\frac{1}{2}$, para todo $n>n_{0}$, vale que

$$
f_{n}(z) \neq-1, \text { se } n>n_{0} \text { e } z \in K
$$

Pelo Teste M de Weierstrass, a série $\sum_{n=n_{0}+1}^{\infty}\left|f_{n}(z)\right|$ converge uniformemente em $K$. Aplicando as proposições A.3 e A.4, a convergência pontual de $\sum_{n=n_{0}+1}^{\infty}\left|f_{n}(z)\right|$ implica que

$$
\prod_{n=n_{0}+1}^{\infty}\left(1+f_{n}(z)\right) \text { existe e é não nulo. }
$$

Sejam $P_{n}=\prod_{k=n_{0}+1}^{n}\left(1+f_{k}\right)$, onde $n>n_{0}$ e $M=\sum_{n=1}^{\infty} M_{n}$. Então, se $n>n_{0}$ e $z \in K$, pela continuidade da função exponencial, temos a desigualdade

$$
\left|P_{n}(z)\right| \leq \prod_{k=n_{0}+1}^{n}\left(1+\left|f_{k}(z)\right|\right) \leq \prod_{k=n_{0}+1}^{\infty}\left(1+\left|f_{k}(z)\right|\right) \leq \prod_{k=n_{0}+1}^{\infty} \exp \left(\left|f_{k}(z)\right|\right) \leq
$$




$$
\leq \lim _{m \rightarrow \infty} \exp \left(\sum_{k=n_{0}+1}^{m} M_{k}\right)=\exp \left(\sum_{k=n_{0}+1}^{\infty} M_{k}\right) \leq e^{M} .
$$

Se $m<n$, então

$$
P_{n}-P_{m}=\sum_{k=m}^{n-1}\left(P_{k+1}-P_{k}\right)=\sum_{k=m}^{n-1}\left[\left(1+f_{k+1}\right) P_{k}-P_{k}\right]=\sum_{k=m}^{n-1} f_{k+1} P_{k} .
$$

Assim, se $m, n>n_{0}$, com $m<n$ e $z \in K$, temos

$$
\left|P_{n}(z)-P_{m}(z)\right| \leq \sum_{k=m}^{n-1}\left|P_{k}(z)\right|\left|f_{k+1}(z)\right| \leq e^{M} \varepsilon .
$$

Segue disso que a sequência

$$
\left(P_{n}\right)_{n \geq n_{0}}=\left(\prod_{k=n_{0}+1}^{n}\left(1+f_{k}\right)\right)_{n \geq n_{0}}
$$

converge uniformemente à função $F$ sem zeros em $K$, dada por

$$
F(z)=\prod_{n=n_{0}+1}^{\infty}\left(1+f_{n}(z)\right), \text { para todo } z \in K .
$$

Portanto, por definição, temos que $f(z)=\prod_{n=1}^{\infty}\left(1+f_{n}(z)\right)$ converge uniformemente em $K$.

Ainda, para todo $z \in K$, vale a igualdade

$$
f(z)=\left[1+f_{1}(z)\right] \cdots\left[1+f_{n_{0}}(z)\right] F(z) .
$$

Finalmente, se $f\left(z_{0}\right)=0$, existe $m \in\left\{1,2, \ldots, n_{0}\right\}$ tal que $f_{m}\left(z_{0}\right)=-1$.

Como consequência do corolário 3.48 (convergência compacta de funções analíticas) e da demonstração do lema A.6, temos o corolário abaixo.

Corolário A.7. Sejam $\Omega \subset \mathbb{C}$ aberto e $\left(f_{n}\right)$ uma sequência de funções analíticas definidas em $\Omega$ e com valores em $\mathbb{C}$. Suponhamos que para cada compacto $K \subset \Omega$ vale que $\sum_{n=1}^{\infty} M_{n}<\infty$, onde $\left\|f_{n}\right\|_{K}=M_{n}$ e $n \in \mathbb{N}$. Nestas condições, a função

$$
f(z)=\prod_{n=1}^{\infty} f_{n}(z)
$$

converge compactamente em $\Omega$ e f é analítica em $\Omega$. Ainda mais, se $z_{0}$ é um zero de $f$, então $z_{0}$ é zero de somente uma quantidade finita de funções $f_{n}$ e a multiplicidade de $z_{0}$ como zero de $f$ é a soma das multiplicidades de $z_{0}$ como zero das $f_{n^{\prime}}$. 
Demonstração. Seja $K \subset \Omega$ um compacto. Pelo lema A.6 a função definida em $\Omega$ por

$$
f(z)=\prod_{n=1}^{\infty} f_{n}(z)
$$

converge uniformemente em $K$.

Dado $z_{0} \in \Omega$, consideremos $r>0$ tal que $D\left(z_{0} ; r\right) \subset \Omega$. Pela demonstração do lema A.6, existe $n_{0} \in \mathbb{N}$ tal que

$$
f(z)=f_{1}(z) f_{2}(z) \cdots f_{n_{0}}(z) \prod_{n=n_{0}+1}^{\infty} f_{n}(z), \text { para todo } z \in D\left(z_{0} ; r\right)
$$

e $\prod_{n=n_{0}+1}^{\infty} f_{n}(z)$ é limite uniforme de funções analíticas em $D\left(z_{0} ; r\right)$.

Segue do corolário 3.48 que $\prod_{n=n_{0}+1}^{\infty} f_{n}(z)$ é analítica em $B(a ; r)$. Portanto, $f$ é analítica em $B\left(z_{0} ; r\right)$ e, consequentemente em $\Omega$.

Se $z_{0} \in Z(f)$, então existe $m \in\left\{1,2, \ldots, n_{0}\right\}$ tal que $f_{m}\left(z_{0}\right)=0$ e, é trivial verificarmos que a multiplicidade de $z_{0}$ como zero de $f$ é a soma das multiplicidades de $z_{0}$ como zero das funções $f_{n^{\prime} s}$.

Definição A.8. Um fator elementar é uma das seguintes funções

$$
\begin{gathered}
E_{0}(z)=1-z, \\
E_{p}(z)=(1-z) \exp \left(z+\frac{z^{2}}{2}+\cdots+\frac{z^{p}}{p}\right), \text { se } p \geq 1 .
\end{gathered}
$$

Os fatores elementares são utilizados para obtermos funções com zeros prescritos de multiplicidade prescrita.

Lema A.9. Se $|z| \leq 1$, então $\left|1-E_{p}(z)\right| \leq|z|^{p+1}$, para todo $p \in \mathbb{N}$.

Demonstração. Se $p=0$, temos que

$$
\left|1-E_{0}(z)\right|=|z|, \text { para todo } z \in \mathbb{C} .
$$

Caso $p>0$, seja $E_{p}(z)=1+\sum_{n=1}^{\infty} a_{n} z^{n}$ a série de Taylor de $E_{p}$ em torno da origem.

Logo, dado $z \in \mathbb{C}$, temos que

$$
\begin{gathered}
E_{p}^{\prime}(z)=\sum_{k=1} k a_{k} z^{k-1}=-\exp \left(z+\frac{z^{2}}{2}+\cdots+\frac{z^{p}}{p}\right)+(1-z)\left(1+z+\cdots+z^{p-1}\right) \exp \left(z+\frac{z^{2}}{2}+\cdots+\frac{z^{p}}{p}\right)= \\
=-z^{p} \exp \left(z+\frac{z^{2}}{2}+\cdots+\frac{z^{p}}{p}\right) .
\end{gathered}
$$

Comparando os coeficientes, temos que $a_{1}=a_{2}=\cdots=a_{p}=0$ e $a_{k}<0\left(\left|a_{k}\right|=-a_{k}\right)$, se $k \geq p+1$. 


$$
\begin{aligned}
& \text { Sabendo que } E_{p}(z)=1+\sum_{n=p+1}^{\infty} a_{n} z^{n} \text {, se }|z| \leq 1 \text {, obtemos a desigualdade } \\
& \begin{array}{c}
\left|1-E_{p}(z)\right|=\left|\sum_{n=p+1}^{\infty} a_{n} z^{n}\right|=\left|z^{p+1} \sum_{n=p+1}^{\infty} a_{n} z^{n-(p+1)}\right| \leq|z|^{p+1} \sum_{n=p+1}^{\infty}\left|a_{n}\right|=-|z|^{p+1} \sum_{n=p+1}^{\infty} a_{n}= \\
=|z|^{p+1}\left(1-E_{p}(1)\right)=|z|^{p+1} .
\end{array}
\end{aligned}
$$

Veremos a seguir uma condição suficiente para que um produto infinito de fatores elementares defina uma função inteira. Antes, notemos que dado $a \in \mathbb{C}$ não nulo, a função $E_{p}\left(\frac{z}{a}\right)$, com $p \geq 0$, tem um zero simples em $z=a$ e este é seu único zero.

Teorema A.10. Seja $\left(a_{n}\right)$ uma sequência em $\mathbb{C}$ tal que $\lim _{n \rightarrow \infty}\left|a_{n}\right|=\infty$ e $a_{n} \neq 0$ (logo, nenhum valor da sequência é repetido uma quantidade infinita de vezes). Se ( $\left.p_{n}\right)$ é uma sequência qualquer de naturais tais que

$$
\sum_{n=1}^{\infty}\left(\frac{r}{\left|a_{n}\right|}\right)^{p_{n}+1}<\infty
$$

para todo $r>0$, então o produto

$$
f(z)=\prod_{n=1}^{\infty} E_{p_{n}}\left(\frac{z}{a_{n}}\right)
$$

converge compactamente em $\mathbb{C}$. A função $f$ é inteira com zeros somente nos pontos $a_{n}$. Se $z_{0}$ ocorre $m$ vezes na sequência $\left(a_{n}\right)$, então $z=z_{0}$ é um zero de ordem $m$ de $f$. Mais ainda, se $p_{n}=n-1$, com $n \geq 1$, então A.2 está satisfeita.

Demonstração. Suponhamos que $\left(p_{n}\right)$ é uma sequência satisfazendo A.2 e consideremos $K \subset \Omega$ compacto.

Seja $r>0$ tal que $K \subset B(0 ; r)$. Como $\lim _{n \rightarrow \infty}\left|a_{n}\right|=\infty$, existe $n_{0} \in \mathbb{N}$ tal que $\left|a_{n}\right| \geq r$, se $n \geq n_{0}$.

Pelo lema A.9, temos que

$$
\left|1-E_{p_{n}}\left(\frac{z}{a_{n}}\right)\right| \leq\left|\frac{z}{a_{n}}\right|^{p_{n}+1} \leq\left(\frac{r}{\left|a_{n}\right|}\right)^{p_{n}+1}, \text { se }|z|<r \text { e } n \geq n_{0} .
$$

Aplicando o corolário A.7, temos a convergência uniforme sobre os compactos de $\mathbb{C}$ do produto

$$
\prod_{n=1}^{\infty} E_{p_{n}}\left(\frac{z}{a_{n}}\right)
$$

e mais, que $f$ é inteira com zeros somente nos pontos $a_{n}$. Ainda mais, se $z_{0}$ ocorre $m$ vezes na sequência $\left(a_{n}\right)$, então $z_{0}$ é um zero de ordem $m$ de $f$.

Para finalizar, basta notarmos que para todo $r>0$ existe $m_{0} \in \mathbb{N}$ tal que $\left|a_{n}\right|>2 r$, se $n \geq m_{0}$. Segue então que $\left(\frac{r}{\left|a_{n}\right|}\right)<\frac{1}{2}$, para $n \geq m_{0}$.

Logo, se $p_{n}=n-1$, temos

$$
\sum_{n=m_{0}}^{\infty}\left(\frac{r}{\left|a_{n}\right|}\right)^{p_{n}+1} \leq \sum_{n=m_{0}}^{\infty}\left(\frac{1}{2}\right)^{n}<\infty,
$$


e portanto, A.2 está satisfeita.

A proposição abaixo decorre trivialmente do Princípio dos zeros Isolados, Princípio do Prolongamento Analítico e da conexidade de $\mathbb{C}$ (vide corolário 4.34 - p. 69), porém, optamos por demonstrá-la.

Proposição A.11. Sejam $f$ e $g$ duas funções inteiras, não nulas, com os mesmo zeros (isto é, $Z(f)=Z(g)$ ), cada zero com a mesma multiplicidade (logo, $\nu(f ; a)=\nu(g ; a)$, para todo $a \in \mathbb{C})$. Então, existe uma função inteira $h$ satisfazendo

$$
f(z)=g(z) e^{h(z)}, \text { para todo } z \in \mathbb{C}
$$

Demonstração. Sejam $W=Z(f)$ e $a \in W$. Pelo Princípio dos Zeros Isolados existem uma bola aberta centrada em $a$, denotada por $V_{a}$, um número $k \geq 1$ e funções $f_{a}, g_{a} \in \mathcal{A}(\mathbb{C})$ que não se anulam em $V_{a}$ tais que

$$
f(z)=(z-a)^{k} f_{a}(z) \text { e } g(z)=(z-a)^{k} g_{a}(z) \text {, para todo } z \in \mathbb{C} .
$$

Consideremos a função $F$ dada por

$$
F(z)=\left\{\begin{array}{l}
\frac{f(z)}{g(z)}, \text { se } z \in \mathbb{C} \backslash W, \\
\frac{f_{z}(z)}{g_{z}(z)}, \text { se } z \in W .
\end{array}\right.
$$

Temos que $F$ está bem definida e é analítica em $\mathbb{C}$. De fato, o conjunto $\mathbb{C} \backslash W$ é aberto e $f \in \mathcal{A}(\mathbb{C} \backslash W)$. Ainda, para cada $a \in W$, temos

$$
F(z)=\frac{f_{a}(z)}{g_{a}(z)} \text {, para todo } z \in V_{a} .
$$

A função $F$ não se anula. Logo, existe $h \in \mathcal{A}(\Omega)$ tal que $F(z)=e^{h(z)}$, para todo $z \in \mathbb{C}$.

Finalmente, pelo Princípio do Prolongamento Analítico, dado que $f(z)=g(z) e^{h(z)}$ em $\mathbb{C} \backslash W$, temos o desejado.

A fim de tornar claro o enunciado do Teorema da fatoração de Weierstrass, façamos o seguinte comentário. Dada $f$ uma função inteira não nula, dizemos que $\left(a_{n}\right)$ é a sequência dos seus zeros não nulos contados com multiplicidade se $Z(f) \backslash\{0\}=\left\{a_{n}: n \in \mathbb{N}\right\}$ ( $f$ tem uma quantidade enumerável infinita de zeros não nulos) e se $z_{0} \in Z(f)$ e $a: \mathbb{N} \rightarrow \mathbb{C}$ é tal que $a(n)=a_{n}$, para todo $n \in \mathbb{N}$, então

$$
\nu\left(f ; z_{0}\right)=\left|a^{-1}\left(z_{0}\right)\right|,
$$

onde $\left|a^{-1}\left(z_{0}\right)\right|$ denota a cardinalidade do conjunto $a^{-1}\left(z_{0}\right)$.

A seguir iremos demonstrar o Teorema da Fatoração de Weierstrass para funções inteiras (no sentido de Weierstrass). Todos os resultados utilizados na demonstração são obtidos sem o uso da teoria de integração. 
Teorema A.12 (Teorema da Fatoração de Weierstrass). Seja $f$ uma função inteira não nula $e\left(a_{n}\right)_{n \geq 1}$ a sequência dos seus zeros não nulos contados com suas respectivas multiplicidades. Se $f$ tem um zero em $z=0$ de ordem $m \geq 0$, então existe uma função inteira $g$ e uma sequência de números naturais $\left(p_{n}\right)_{n \geq 1}$ tais que

$$
f(z)=z^{m} e^{g(z)} \prod_{n=1}^{\infty} E_{p_{n}}\left(\frac{z}{a_{n}}\right), \text { para todo } z \in \mathbb{C} .
$$

Demonstração. Por hipótese, $f$ tem uma quantidade enumerável de zeros. Ainda, como $f$ é inteira e não nula, para cada $r>0$, a função $f$ tem uma quantidade finita de zeros em $D(0 ; r)$. Logo, $\lim _{n \rightarrow \infty}\left|a_{n}\right|=\infty$.

Pelo teorema A.10 podemos escolher naturais $p_{n}$ de modo que a função inteira definida por

$$
h(z)=z^{m} \prod_{n=1}^{\infty} E_{p_{n}}\left(\frac{z}{a_{n}}\right),
$$

tem os mesmos zeros de $f$, com respectivas multiplicidades iguais.

Sendo $f$ e $h$ funções inteiras, segue da proposição A.11 que existe $g$ inteira tal que $f(z)=$ $e^{g(z)} h(z)$, para todo $z \in \mathbb{C}$.

\section{A.2 O Pequeno Teorema de Picard}

O resultado desta seção é surpreendente, além de mais geral que o Teorema de Liouville (p. 36). Existe uma versão mais forte do Pequeno Teorema de Picard conhecida como Grande Teorema de Picard.

As provas mais conhecidas de tal teorema utilizam resultados que decorrem do corolário 4.34 (p. 69) e este, por sua vez, é obtido através da existência de primitivas de funções holomorfas e, portanto, através da teoria de integração (ver [3]).

Dada uma função $f$, dizemos que sua imagem omite o valor $\omega$ se $\omega \notin \operatorname{Imagem}(f)$. O Pequeno Teorema de Picard nos diz que se a imagem de uma função inteira $f$ omite dois valores então $f$ é constante, ou ainda, se $f$ é uma função inteira não constante, então temos $\operatorname{Imagem}(f)=\mathbb{C}$ ou $\operatorname{Imagem}(f)=\mathbb{C} \backslash\{p\}$, para algum $p \in \mathbb{C}$. Observemos que este é o melhor resultado possível, pois a função exponencial complexa é inteira não constante e $\exp (\mathbb{C})=\mathbb{C}^{*}$.

Antes de provarmos o principal resultado desta seção desenvolveremos alguns lemas auxiliares. As provas apresentadas são as clássicas, porém, as afirmações que decorrem do uso de alguma integral serão modificadas para que usemos apenas os resultados obtidos neste trabalho.

O Teorema de Bloch nos traz informações sobre a imagem de funções analíticas em bolas. Não são conhecidas provas simples de tal resultado, mas nos esforçamos para sermos claros.

Lema A.13. Seja $f \in \mathcal{A}(B(0 ; 1))$. Se $f(0)=0, f^{\prime}(0)=1$ e $|f(z)| \leq M$, para todo $z \in B(0 ; 1)$, então temos $M \geq 1 e$

$$
B\left(0 ; \frac{1}{6 M}\right) \subset f(B(0 ; 1))
$$


Demonstração. Considere $f(z)=\sum_{n=1}^{\infty} a_{n} z^{n}$ a série de Taylor de $f$ em torno da origem. Pelo Lema de Schwarz (p. 44) temos que $\left|a_{n}\right| \leq M$, para todo $n \geq 1$ e portanto $M \geq\left|a_{1}\right|=1$.

Sejam $w \in \mathbb{C}$ com $|w|<6 M^{-1}$ e $g(z)=f(z)-w$, onde $|z|<1$. É suficiente mostrarmos que $g$ tem um zero, para isto, aplicaremos o Teorema de Rouché (p. 65) às funções $f$ e $g$ na bola $B\left(0 ; 4 M^{-1}\right)$.

Se $|z|=4 M^{-1}$, então

$$
|f(z)| \geq|z|-\sum_{n=2}^{\infty}\left|a_{n} z^{n}\right| \geq \frac{1}{4 M}-\sum_{n=2}^{\infty} M\left(\frac{1}{4 M}\right)^{n} \geq \frac{1}{4 M}-\frac{1}{16 M-4} \geq \frac{1}{6 M} .
$$

Consequentemente temos

$$
|f(z)-g(z)|=|w|<\frac{1}{6 M} \leq|f(z)|, \text { se }|z|=\frac{1}{4 M},
$$

mas como $f(0)=0$, a função $g$ se anula em algum $z_{0} \in B(0 ; 1)$.

Como consequência, temos o corolário a seguir.

Corolário A.14. Se $f \in \mathcal{A}(B(0 ; r)), f(0)=0,\left|f^{\prime}(0)\right|=c>0$ e $|f(z)| \leq M$, para $|z|<r$, então

$$
B\left(0 ; \frac{r^{2} c^{2}}{6 M}\right) \subset f(B(0 ; r)) .
$$

Demonstração. Considere a função

$$
g(z)=\frac{f(r z)}{r f^{\prime}(0)}, \operatorname{com}|z|<1 .
$$

Então $g$ está nas condições do lema anterior e $|g(z)| \leq \frac{M}{r c}$ em $B(0 ; 1)$. Logo,

$$
B\left(0 ; \frac{c r}{6 M}\right) \subset g(B(0 ; 1))
$$

Para finalizarmos, basta notarmos que

$$
g(B(0 ; 1))=\frac{1}{r f^{\prime}(0)} f(B(0 ; r))
$$

O seguinte lema é bem simples, mas será importante para provarmos o Teorema de Bloch.

Lema A.15. Seja $f$ analítica em um aberto contendo $D(0 ; 1)$. Então a função $M:[0,1] \rightarrow \mathbb{R}$, dada por $M(r)=\max _{|z|=r}|f(z)|$, é contínua.

Demonstração. Seja $\varepsilon>0$. Como $f$ é uniformemente contínua em $D(0 ; 1)$, existe $\delta>0$ tal que

$$
|f(z)-f(w)|<\varepsilon, \text { se }|z-w|<\delta \text { e } z, w \in D(0 ; 1) .
$$

Seja $r \in[0,1]$ arbitrário e fixado. 
Dados

$$
\omega \in S^{1} \text { e } s \in(r-\delta, r+\delta) \cap[0,1]
$$

temos que $r \omega, s \omega \in D(0 ; 1)$ e $|s \omega-r \omega|=|s-r|<\delta$. Logo, $|f(s \omega)-f(r \omega)|<\varepsilon$ e, portanto

$$
|f(r \omega)|-\varepsilon \leq|f(s \omega)| \leq|f(r \omega)|+\varepsilon
$$

Variando $\omega$ em $S^{1}$, obtemos que $|M(s)-M(r)|<\varepsilon$, se $|r-s|<\delta$ e $r, s \in[0,1]$. Portanto, $M$ é contínua em $[0,1]$.

É possível demonstrar um versão mais forte do Teorema de Bloch (ver [23]), porém, para nossos propósitos, esta é suficiente.

Teorema A.16 (Bloch). Seja $f$ analítica em um aberto contendo $D(0 ; 1)$, com $f(0)=0$ e $f^{\prime}(0)=$ 1. Então, $f(D(0 ; 1))$ contém uma bola (não necessariamente centrada na origem) de raio

$$
\frac{1}{48}
$$

Demonstração. Dividiremos a prova em 3 partes.

Passo 1. Para cada $r \in[0,1]$, sejam $N(r)=\max _{|z|=r}\left|f^{\prime}(z)\right|$ e $h(r)=(1-r) N(r)$. Como $f^{\prime}$ é analítica no mesmo subconjunto que $f$, segue do lema A.15 que $N$ é contínua em $[0,1]$ e, portanto $h$ também, com $h(0)=\left|f^{\prime}(0)\right|=1$ e $h(1)=0$.

Seja $r_{0}=\sup \{r \in[0,1]: h(r)=1\}$. Pela continuidade de $h$ tal supremo é assumido. Logo, $h\left(r_{0}\right)=1$ e $r_{0}<1$.

É válido que

$$
h(r)<1, \text { se } r \in\left(r_{0}, 1\right],
$$

caso contrário existe $r_{1} \in\left(r_{0}, 1\right]$ tal que $h\left(r_{1}\right)>1$. Como $h$ é contínua, pelo Teorema do Valor Intermediário, existe $r_{2} \in\left(r_{1}, 1\right) \operatorname{com} h\left(r_{2}\right)=1$. Mas $r_{2}>r_{0}$, o que contraria a definição de $r_{0}$.

Ainda, pela definição de $N\left(r_{0}\right)$, existe $a \in \mathbb{C}$ tal que

$$
|a|=r_{0} \quad \text { e }\left|f^{\prime}(a)\right|=N\left(r_{0}\right)
$$

Como $h\left(r_{0}\right)=1$, pela definição de $h$, temos que

$$
\left|f^{\prime}(a)\right|=N\left(r_{0}\right)=\frac{1}{1-r_{0}} \neq 0
$$

Passo 2. Seja $\rho_{0}=\frac{1-r_{0}}{2}$. Se $|z|<\rho_{0}+r_{0}<1$, pelo Princípio do Módulo Máximo (p. 36) aplicado à função $f^{\prime}$ e a relação A.3, segue que

$$
\left|f^{\prime}(z)\right| \leq K\left(\rho_{0}+r_{0}\right)=h\left(\rho_{0}+r_{0}\right)\left[1-\left(\rho_{0}+r_{0}\right)\right]^{-1}<\left[1-\left(\rho_{0}+r_{0}\right)\right]^{-1}=\frac{1}{\rho_{0}} .
$$

Pela Desigualdade de Gutzmer-Parseval (p. 41) aplicada à função $f^{\prime}$ na bola $B\left(0 ; \rho_{0}+r_{0}\right)$ e a 
desigualdade em A.4, temos que

$$
\sum_{n=1}^{\infty} n^{2}\left|a_{n}\right|^{2} \rho_{0}^{2 n-2} \leq \frac{1}{\rho_{0}^{2}} .
$$

Passo 3. Escrevendo $f(z)=\sum_{n=0}^{\infty} a_{n}(z-a)^{n}$ em $B\left(a ; \rho_{0}\right) \subset B(0 ; 1)$, vale que

$$
f(z+a)-f(a)=\sum_{n=1}^{\infty} a_{n} z^{n}, \text { se }|z|<\rho_{0}
$$

Considerando a função $g(z)=f(z+a)-f(a)$, com $|z|<\rho_{0}$, temos que $g(0)=0$ e $\left|g^{\prime}(0)\right|=$ $\left|f^{\prime}(a)\right|=\frac{1}{2 \rho_{0}}>0$. Ainda, se $|z|<\rho_{0}$, obtemos

$$
\begin{aligned}
|g(z)| & =|z|\left|\sum_{n=1}^{\infty} \frac{1}{n} n a_{n} z^{n-1}\right| \leq|z| \sum_{n=1}^{\infty} \frac{1}{n} n\left|a_{n}\right||z|^{n-1} \leq \rho_{0} \sum_{n=1}^{\infty} \frac{1}{n} n\left|a_{n}\right| \rho_{0}^{n-1} \leq \\
& \leq \rho_{0}\left(\sum_{n=1}^{\infty} \frac{1}{n^{2}}\right)^{\frac{1}{2}}\left(\sum_{n=1}^{\infty} n^{2}\left|a_{n}\right|^{2} \rho_{0}^{2 n-2}\right)^{\frac{1}{2}} \leq \rho_{0}\left(\sum_{n=1}^{\infty} \frac{1}{n^{2}}\right)^{\frac{1}{2}} \frac{1}{\rho_{0}}<2 .
\end{aligned}
$$

Decorre do corolário A.14 que $g\left(B\left(0 ; \rho_{0}\right)\right)$ contém uma bola centrada na origem de raio

$$
R=\frac{\rho_{0}^{2}\left(\frac{1}{2 \rho_{0}}\right)^{2}}{12}=\frac{1}{48} .
$$

Portanto, $f(D(0 ; 1))$ contém a bola de centro $f(a)$ e raio $\frac{1}{48}$

Corolário A.17. Seja $f$ analítica em um aberto contendo $D(0 ; r)$ com $f^{\prime}(0) \neq 0$. Então, $f(D(0 ; r))$ contém uma bola (não necessariamente centrada na origem) de raio

$$
\frac{r\left|f^{\prime}(0)\right|}{48} \text {. }
$$

Demonstração. Basta aplicarmos o Teorema de Bloch a função dada por

$$
g(z)=\frac{f(r z)-f(0)}{r f^{\prime}(0)} \text {, onde } z \in D(0 ; r)
$$

e observarmos que se $g(B(0 ; r))$ contém a bola de centro $c$ e raio $\frac{1}{48}$, então $f(D(0 ; r))$ contém a bola de centro $c f^{\prime}(0) r+f(0)$ e raio $\frac{r\left|f^{\prime}(0)\right|}{48}$.

A função cosseno hiperbólico é definida por

$$
\cosh (z)=\frac{e^{z}+e^{-z}}{2}, \text { para todo } z \in \mathbb{C}
$$


Lema A.18. Sejam $\Omega$ aberto simplesmente conexo e $f \in \mathcal{A}(\Omega)$ omitindo os valores 0 e 1 . Então existe $g \in \mathcal{A}(\Omega)$ tal que

$$
f(z)=-\exp [i \pi \cosh (2 g(z))], \text { para todo } z \in \Omega \text {. }
$$

Demonstração. Pelo corolário 4.34 (existência de logaritmo e raiz $n$-ésima de funções analíticas p. 69), existe $\varphi \in \mathcal{A}(\Omega)$ tal que $f=e^{\varphi}$. No que segue $\varphi$ está fixada.

Dada a periodicidade da exponencial complexa, a continuidade de $\varphi$ e a conexidade de $\Omega$, nosso problema estará resolvido se determinarmos $g \in \mathcal{A}(\Omega)$ tal que

$$
\varphi(z)=i \pi \cosh (2 g(z))+i \pi, \text { para todo } z \in \Omega .
$$

Definindo $\Phi \equiv \frac{\varphi}{i \pi}$, podemos reescrever a equação A.5 como

$$
\Phi(z)=\cosh (2 g(z))+1, \text { para todo } z \in \Omega
$$

Pela definição da função cosseno hiperbólico e a equação (A.6), devemos determinar $g \in \mathcal{A}(\Omega)$ tal que

$$
2 \Phi=\left(e^{g}+e^{-g}\right)^{2} .
$$

Pelo corolário 4.34, é suficiente determinarmos $G \in \mathcal{A}(\Omega)$, sem zeros, satisfazendo

$$
2 \Phi(z)=\left(G(z)+\frac{1}{G(z)}\right)^{2}, \text { para todo } z \in \Omega
$$

A função $f$ omite o valor 1 . Logo, a função $\Phi$ omite qualquer inteiro par, caso contrário, se existissem $a \in \Omega$ e $n \in \mathbb{Z}$ tais que $\Phi(a)=2 n$, então

$$
f(a)=\exp (\varphi(a))=\exp (i \pi \Phi(a))=\exp (2 i \pi n)=1
$$

o que não pode ocorrer.

Consideremos $\Psi \in \mathcal{A}(\Omega)$ (existência garantida pelo corolário 4.34) tal que $2 \Phi=\Psi^{2}$ em $\Omega$. Portanto, nosso problema será resolvido se determinarmos $G$ tal que

$$
G+\frac{1}{G}=\Psi
$$

isto é, $G$ satisfazendo

$$
G(z)^{2}-G(z) \Psi(z)+1=0, \text { para todo } z \in \Omega .
$$

É claro que se $G$ for solução da equação A.7, então $G$ não se anula em $\Omega$. Observemos ainda que resolver essa equação é equivalente a determinar uma solução da seguinte equação

$$
(2 G-\Psi)^{2}+\left(4-\Psi^{2}\right)=0 .
$$

Dado que $\Psi^{2}-4$ não se anula (pois $\Psi^{2}-4=2 \Phi-4$ e $\Phi$ não assume valores pares), tal função possui uma raiz quadrada (existência garantida pelo corolário 4.34) que vamos fixar e denotar por 
$\sqrt{\Psi^{2}-4}$. Logo, a seguinte função analítica definida em $\Omega$

$$
G=\frac{\Psi-\sqrt{\Psi^{2}-4}}{2}
$$

é solução da equação A.7.

Corolário A.19. Sejam $\Omega$ aberto simplesmente conexo e $f, g \in \mathcal{A}(\Omega)$ como no lema A.18. Nessas condições, $g(\Omega)$ não contém nenhuma bola de raio 1 .

Demonstração. Vamos mostrar que $g(\Omega)$ não contém nenhum dos pontos do conjunto

$$
A=\left\{ \pm \ln (\sqrt{n}+\sqrt{n-1})+\frac{i m \pi}{2}: n \in \mathbb{N}^{*}, m \in \mathbb{Z}\right\}
$$

Sejam $n \in \mathbb{N}^{*}$ e $m \in \mathbb{Z}$. Se existir $a \in \Omega$ tal que

$$
g(a)= \pm \ln (\sqrt{n}+\sqrt{n-1})+\frac{i m \pi}{2} .
$$

Então,

$$
\begin{gathered}
2 \cosh (2 g(a))=e^{2 g(a)}+e^{-2 g(a)}=e^{i m \pi}(\sqrt{n}+\sqrt{n-1})^{ \pm 2}+e^{-i m \pi}(\sqrt{n}+\sqrt{n-1})^{\mp 2}= \\
=(-1)^{m}\left[(\sqrt{n}+\sqrt{n-1})^{2}+(\sqrt{n}-\sqrt{n-1})^{2}\right]=2(-1)^{m}(2 n-1) .
\end{gathered}
$$

Assim, $f(a)=-\exp \left((-1)^{m} i \pi(2 n-1)\right)=1$, contra nossa hipótese.

Notemos que tais pontos formam um engradado de retângulos no plano e cada retângulo tem altura igual a $\frac{\pi}{2}<\sqrt{3}$ e largura dada por

$$
\ln (\sqrt{n+1}+\sqrt{n})-\ln (\sqrt{n}+\sqrt{n-1}) .
$$

Como $\sqrt{n+1}+\sqrt{n} \leq 2 \sqrt{n+1}$ e $\sqrt{n}+\sqrt{n-1} \geq 2 \sqrt{n-1}$, se $n \geq 2$, temos que

$$
\ln (\sqrt{n+1}+\sqrt{n})-\ln (\sqrt{n}+\sqrt{n-1}) \leq\left\{\begin{array}{l}
\ln (\sqrt{2}+1)<1, \text { se } n=1 \\
\ln \left(\sqrt{\frac{n+1}{n-1}}\right) \leq \ln (\sqrt{3})<1, \text { se } n \geq 2
\end{array}\right.
$$

Dado $p=\left(p_{1}, p_{2}\right) \in \mathbb{R}^{2}$ arbitrário, existem $n \geq 1$ e $m \in \mathbb{Z}$ tais que

$$
\left(p_{1}, p_{2}\right) \in[\ln (\sqrt{n}+\sqrt{n-1}), \ln (\sqrt{n+1}+\sqrt{n})] \times\left[\frac{m \pi}{2}, \frac{(m+1) \pi}{2}\right] .
$$

Sem perda de generalidade, podemos supor que

$$
\left(p_{1}, p_{2}\right) \in[0, a] \times[0, b]
$$

com $a<1$ e $b<\sqrt{3}$.

Sejam $O=(0,0)$ e $C=\left(\frac{a}{2}, \frac{b}{2}\right)$ pontos do plano, $R_{1}=\left[0, \frac{a}{2}\right] \times\left[0, \frac{b}{2}\right]$ o subretângulo inferior à esquerda, $R_{2}$ o subretângulo inferior à direita, $R_{3}$ e $R_{4}$ os subretângulos superiores. 
Temos que

$$
d(C, O)<\frac{\sqrt{1^{2}+(\sqrt{3})^{2}}}{2}=1 .
$$

Analogamente, a distância entre qualquer vértice do retângulo $R=[0, a] \times[0, b]$ e o ponto $C$ é inferior a 1. Logo, o diâmetro de cada um dos subretângulos é menor que 1.

O ponto $p$ pertence a algum dos subretângulos fechados $R_{1}, R_{2}, R_{3}, R_{4}$ e então a bola $B(p ; 1)$ contém ao menos um destes vértices. Portanto, dado $p \in \mathbb{C}$, existe $q \in A$ tal que $|p-q|<1$. Assim, $g(\Omega)$ não pode conter nenhuma bola de raio 1.

Teorema A.20 (Pequeno Teorema de Picard). Se f é uma função inteira cuja imagem omite dois valores, então $f$ é constante.

Demonstração. Suponhamos que $f$ é não constante e omite os valores distintos $a$ e $b$. Consideremos a função inteira

$$
F=\frac{f-a}{b-a} .
$$

Logo, a imagem de $F$ omite os valores 0 e 1 e, a função $F$ é não constante. Pelo lema A.18, existe uma função inteira $G$ tal que

$$
F=-\exp (i \pi \cosh (2 G))
$$

Pelo corolário A.19, o conjunto $G(\mathbb{C})$ não contém nenhuma bola de raio 1 . Como $G$ é não constante, existe $z_{0} \in \mathbb{C}$ tal que $G^{\prime}\left(z_{0}\right) \neq 0$.

Seja $H=G\left(z+z_{0}\right)$. Então temos que $H^{\prime}(0) \neq 0$. Dado $r>0$ e aplicando o corolário A.17 à função $H$, temos que $H(B(0 ; r))$ contém uma bola de raio

$$
\frac{1}{48} r\left|H^{\prime}(0)\right|
$$

Para $r$ suficientemente grande, tem-se que $H(\mathbb{C})$ contém uma bola de raio 1 e, portanto $G(\mathbb{C})$ também, o que é uma contradição. Logo, a função $G$ é constante e, consequentemente, $f$ é constante. 


\section{Apêndice B}

\section{Apêndice}

O resultado abaixo é conhecido como Propriedade Poligonal do Valor Médio.

Proposição B.1 (Kakutani - Nagamo, Walsh). Sejam $P(z)=\sum_{j=0}^{n} a_{j} z^{j}$ um polinômio complexo não constante e $\omega$ uma raiz primitiva 2 -ésima da unidade. Para cada $z_{0}$ e z complexos, temos

$$
P\left(z_{0}\right)=\frac{1}{2 n} \sum_{k=0}^{2 n-1} P\left(z_{0}+z \omega^{k}\right)
$$

Demonstração. Dividiremos a prova em dois casos.

Primeiro caso. Suponhamos $z_{0}=0$.

Consideremos os $2 n$-polinômios $P_{k}(z)=P\left(z \omega^{k}\right)$, com $k=0,1, \ldots 2 n-1$.

Observemos que valem as igualdades

$$
\sum_{k=0}^{2 n-1} \omega^{k j}=\left\{\begin{array}{l}
2 n, \text { se } j=0 \\
\frac{1-\omega^{2 n j}}{1-\omega^{j}}=0, \text { se } 1 \leq j \leq n .
\end{array}\right.
$$

Somando os $2 n$-polinômios, obtemos

$$
\sum_{k=0}^{2 n-1} P_{k}(z)=\sum_{k=0}^{2 n-1} \sum_{j=0}^{n} a_{j} z^{j} \omega^{k j}=2 n a_{0}+\sum_{j=1}^{n} a_{j} z^{j}\left(\sum_{k=0}^{2 n-1} \omega^{k j}\right)=2 n a_{0} .
$$

Logo,

$$
P(0)=\frac{1}{2 n} \sum_{k=0}^{2 n-1} P\left(0+z \omega^{k}\right)
$$

Segundo caso. Suponhamos $z_{0} \in \mathbb{C}$ arbitrário.

Considere o polinômio $Q(z)=P\left(z+z_{0}\right)$. Pelo caso anterior, temos

$$
P\left(z_{0}\right)=Q(0)=\frac{1}{2 n} \sum_{k=0}^{2 n-1} Q\left(z \omega^{k}\right)=\frac{1}{2 n} \sum_{k=0}^{2 n-1} P\left(z_{0}+z \omega^{k}\right) .
$$


Teorema B.2 (Princípio do Módulo Máximo). Seja $P(z)=\sum_{j=0}^{n} a_{j} z^{j}$ um polinômio complexo não constante. Então, $|P|$ não tem máximo local.

Demonstração 1. Suponhamos sem perda de generalidade que $z_{0}=0$ é ponto de máximo local. Por hipótese, existe $R>0$ tal que $|P(z)| \leq|P(0)|$, se $|z|<R$.

Sejam $z \in B(0 ; R)$ e $\omega$ raiz primitiva $2 n$-ésima da unidade. Pela proposição B.1, tem-se que

$$
P(0)=\frac{1}{2 n} \sum_{k=0}^{2 n-1} P\left(z \omega^{k}\right)
$$

Logo, para $z$ e $\omega$ como acima

$$
|P(0)| \leq \frac{1}{2 n} \sum_{k=0}^{2 n-1}\left|P\left(z \omega^{k}\right)\right| \leq \frac{1}{2 n}(|P(z)|+(2 n-1)|P(0)|) \leq|P(0)| .
$$

Consequentemente, temos $|P(z)|=|P(0)|$, se $|z|<R$.

Dado $z=x \in[0, R)$, temos

$$
\left|a_{0}\right|^{2}=|P(0)|^{2}=P(z) \overline{P(z)}=\sum_{j=0}^{n}\left|a_{j}\right| x^{2 j}+2 \sum_{0 \leq l<k \leq n} \operatorname{Re}\left(a_{l} x^{l+k} \bar{a}_{k}\right) .
$$

Portanto,

$$
\sum_{j=1}^{n}\left|a_{j}\right| x^{2 j}+2 \sum_{0 \leq l<k \leq n} \operatorname{Re}\left(a_{l} \bar{a}_{k}\right) x^{l+k}+\left|a_{n}\right|^{2} x^{2 n}=0, \text { para todo } x \in[0, R) .
$$

Pelo TFA e o algoritmo da divisão para polinômios, um polinômio de grau $m \geq 1$ tem no máximo $m$ raízes, donde segue que $\left|a_{n}\right|=0$.

Como $\left|a_{n}\right|=0$, temos que o polinômio

$$
P_{1}(z)=\sum_{j=1}^{n-1}\left|a_{j}\right| z^{2 j}+2 \sum_{0 \leq l<k \leq n} \operatorname{Re}\left(a_{l} z^{l+k} \bar{a}_{k}\right)
$$

tem o coeficiente de grau $2 n-1$ também nulo. Obtemos novamente, pelo TFA, que $\left|a_{n-1}\right|=0$. Prosseguindo com tal argumento, tem-se que

$$
\left|a_{n}\right|=\left|a_{n-1}\right|=\left|a_{n-2}\right|=\ldots=\left|a_{1}\right|=0 .
$$

Concluímos assim que $P$ é constante igual à $a_{0}$.

Demonstração 2. Suponhamos sem perda de generalidade que $z_{0}=0$ é ponto de máximo local. Seja $r>0$ tal que $|P(z)| \leq|P(0)|=\left|a_{0}\right|$, se $|z| \leq r$. Pela desigualdade de Gutzmer-Parseval para polinômios (p. 12), temos

$$
\sum_{j=0}^{n}\left|a_{j}\right|^{2} r^{2 j} \leq\left|a_{0}\right|^{2}
$$


A desigualdade em B.1 implica que $\sum_{j=1}^{n}\left|a_{j}\right|^{2} r^{2 j} \leq 0$. Portanto, $P(z)=a_{0}$ para todo $z \in \mathbb{C}$, o que é uma contradição.

Corolário B.3 (Desigualdades de Cauchy para séries). Seja $f(z)=\sum_{n=0}^{\infty} a_{n}\left(z-z_{0}\right)^{n}$ uma série convergente em $B\left(z_{0} ; \rho\right)$, com $\rho>0$. Considere $0 \leq r<R<\rho$, então, para todo $n \in \mathbb{N}$, valem as desigualdades

$$
\left\|f^{(n)}\right\|_{D\left(z_{0} ; r\right)} \leq \frac{n !}{(R-r)^{n}}\|f\|_{\partial D\left(z_{0} ; R\right)} \quad e \quad\left|a_{n}\right| \leq \frac{\|f\|_{\partial D\left(z_{0} ; R\right)}}{R^{n}}
$$

Demonstração 2. Seja $a \in D\left(z_{0} ; r\right)$. Pelo Teorema de Translação a função dada por $g(z)=f(z+a)$ é analítica em $D\left(0 ; \rho-\left|a-z_{0}\right|\right)$. Pelo lema 3.39 e observando que se $|z|=R-r<\rho-\left|a-z_{0}\right|$, então $z+a \in D\left(z_{0} ; R\right)$, temos

$$
\frac{\left|g^{(n)}(0)\right|}{n !} \leq \frac{\|g\|_{\partial D(0 ; R-r)}}{(R-r)^{n}} \leq \frac{\|f\|_{\partial D\left(z_{0} ; R\right)}}{(R-r)^{n}}
$$

Concluímos então que

$$
\left|f^{(n)}(a)\right| \leq \frac{n !}{(R-r)^{n}}\|f\|_{\partial D\left(z_{0} ; R\right)}
$$

A seguir demonstraremos o Teorema de Representação Local utilizando a função exponencial.

Teorema B.4 (Representação Local). Sejam $f \in \mathcal{A}(\Omega)$ não constante, $z_{0} \in \Omega$ e $m$ a ordem de $z_{0}$ como zero da função $h: z \in \Omega \mapsto f(z)-f\left(z_{0}\right)$. Então, existem um aberto $V$ contendo a origem, uma bola $B(0 ; r)$ e um isomorfismo analítico $\varphi: V \rightarrow B(0 ; r)$, com $\varphi(0)=0$, satisfazendo

$$
f(z)=f\left(z_{0}\right)+\left[\varphi\left(z-z_{0}\right)\right]^{m} \text {, para todo } z \in z_{0}+V .
$$

Ainda mais, dado $w \in B\left(f\left(z_{0}\right) ; r_{m}\right) \backslash\left\{f\left(z_{0}\right)\right\}$, o conjunto $f^{-1}(w) \cap\left(z_{0}+V\right)$ tem $m$ elementos.

Demonstração 2. Observemos inicialmente que podemos supor que $\Omega$ é uma bola centrado em $z_{0}$ e que $z_{0}$ é o único zero de $f(z)-f\left(z_{0}\right)$ na bola. Pelo Princípio dos zeros isolados, podemos escrever $f(z)=f\left(z_{0}\right)+\left(z-z_{0}\right)^{m} g(z)$, com $g$ analítica em $\Omega$ e $g(z) \neq 0$, para todo $z \in \Omega$.

Como consequência da proposição 3.23, segue que $\frac{g^{\prime}}{g} \in \mathcal{A}(\Omega)$. Pelo teorema 3.40 (Teorema de Hurwitz - p. 42) temos que $\frac{g^{\prime}}{g}$ é dada por uma séries de potências convergente em $\Omega$ e, portanto pela proposição 3.26 (primitiva de função analítica em bolas) existe $h \in \mathcal{A}(\Omega)$ tal que $h^{\prime}=\frac{g^{\prime}}{g}$.

Consideremos a função $\psi=g \exp (-h)$. Logo, $\psi^{\prime} \equiv 0$, o que implica que $\psi$ é constante em $\Omega$. Como $g$ é não nula, a função $\psi$ é não nula.

Sejam $b, \lambda \in \mathbb{C}$ tais que $\psi(z)=b$ e $e^{\lambda}=b$. Assim, $g(z)=e^{h(z)+\lambda}$, para todo $z \in \Omega$.

Definamos $\phi\left(z-z_{0}\right)=\left(z-z_{0}\right) e^{\frac{h(z)+\lambda}{m}}$, com $z \in \Omega$. Logo, para todo $z \in \Omega+z_{0}$ temos

$$
f\left(z_{0}\right)+\phi\left(z-z_{0}\right)^{m}=f\left(z_{0}\right)+\left[\left(z-z_{0}\right) e^{\frac{h(z)+\lambda}{m}}\right]^{m}=f\left(z_{0}\right)+\left(z-z_{0}\right)^{m} g(z)=f(z) .
$$


Observemos que $\phi(0)=0$ e $\phi^{\prime}(0)=e^{\frac{h\left(z_{0}\right)+\lambda}{m}} \neq 0$. Para encerrar, basta procedermos como na demonstração dada na seção 3.2.1.

Proposição B.5. Seja $\left(z_{n}\right)$ uma sequência tal que Re $\left(z_{n}\right)>0$, para todo $n \in \mathbb{N}$. Então, a série $\sum_{n=1}^{\infty} \log \left(z_{n}\right)$ converge se e somente se $\prod_{n=1}^{\infty} z_{n}$ converge.

Demonstração. Suponhamos inicialmente que $\sum_{n=1}^{\infty} \log \left(z_{n}\right)$ converge. Seja $\left(s_{n}\right)$ a sequência das suas somas parciais e $s=\lim _{n \rightarrow \infty} s_{n}$. Então, pela continuidade da função exponencial temos $\lim _{n \rightarrow \infty} \exp \left(s_{n}\right)=$ $\exp (s)$. Como, $\exp \left(s_{n}\right)=\prod_{k=1}^{n} z_{n}$, segue

$$
\prod_{n=1}^{\infty} z_{n}=e^{s} \neq 0
$$

Reciprocamente, suponhamos que $\prod_{n=1}^{\infty} z_{n}$ converge. Seja $\left(p_{n}\right)$ a sua sequência dos produtos parciais. Logo, temos $p_{n} \neq 0$, para todo $n \in \mathbb{N}$.

Denotemos

$$
p=\prod_{n=1}^{\infty} z_{n}
$$

Como $p \neq 0$, podemos considerar Log* um ramo do logaritmo definido num aberto $\Omega$ contendo $p$.

Seja $n_{0} \in \mathbb{N}$ tal que $p_{n} \in \Omega$, se $n \geq n_{0}$. Então, se $n \geq n_{0}$, temos que

$$
\exp \left(\log \left(z_{1}\right)+\log \left(z_{2}\right)+\cdots+\log \left(z_{n}\right)\right)=p_{n}=\exp \left(\log ^{*}\left(p_{n}\right)\right)
$$

Logo, para cada $n \geq n_{0}$, existe um único $k_{n} \in \mathbb{N}$ tal que

$$
\log \left(z_{1}\right)+\log \left(z_{2}\right)+\cdots+\log \left(z_{n}\right)=\log ^{*}\left(p_{n}\right)+2 i \pi k_{n}
$$

Pela continuidade da função Log e lembrando que $z_{n} \rightarrow 1$, tem-se que

$$
\log \left(z_{n}\right) \rightarrow 0
$$

Consequentemente, como

$$
\log ^{*}\left(p_{n}\right)-\log ^{*}\left(p_{n-1}\right) \rightarrow 0
$$

e

$$
s_{n}-s_{n-1}=\log \left(z_{n}\right)=\log *\left(p_{n}\right)-\log ^{*}\left(p_{n-1}\right)+2 i \pi\left(k_{n}-k_{n-1}\right),
$$

segue que $k_{n}-k_{n-1} \rightarrow 0$.

Mas, $k_{n} \in \mathbb{N}$ e, portanto existem $k, n_{0} \in \mathbb{N}$ tais que $k_{n}=k$, se $n \geq n_{0}$.

Notemos que

$$
\lim _{n \rightarrow \infty} s_{n}=\log ^{*}(p)+2 i \pi k .
$$


Portanto, a série $\sum_{n=1}^{\infty} \log \left(z_{n}\right)$ converge. 


\section{Referências Bibliográficas}

[1] Bak, J., Newman, D. J., Complex Analysis. Springer - Verlag, New York, 2nd Edition, 1996. 80

[2] Beardon, A. F. Limits, A New Approach to Real Analysis. Springer - Verlag, New York, 1997. 16

[3] Conway, J. B., Function of One Complex Variable. Spring - Verlag, New York, 2nd Edition, 1978. $16,80,88$

[4] Dyer, R. H., Edmunds, D. E. From Real to Complex Analysis. Springer, New York, 2014. 67

[5] Gray, J., On the history of the Riemann mapping theorem. Rendiconti del Circolo Matematico di Palermo, Serie II, (1994), Supplemento (34): 47-94. 72

[6] Haruki, S., On the mean value property of harmonic and complex polynomials. Proc. Japan Acad. Ser. A Math. Sci. 57 (1981) 216-218. https://projecteuclid.org/euclid.pja/euclid.pja/ 119551645312

[7] Knopp, K., Theory and Application of Infinite Series. Blackie and Son Limited, London and Glasgow, 2nd Edition, 1954. 25, 42

[8] Lang, S., Verlag, New York, 2nd Edition, 1985. 25

[9] Leland, K. O., A polynomial approach to topological analysis. Compos. Math. 17 (1965) 291298. 12

[10] Lima, E. L., Análise Real, volume 2. IMPA, Rio de Janeiro, 2004. 1, 2, 3, 58

[11] Lima, E. L., Translated by Jonas Gomes. Fundamental Groups and Covering Spaces. A K Peters, Natick, 3rd Edition, 2003. 57, 58, 64

[12] Moritz, R. E., On Certain Proof's of the Fundamental Theorem of Algebra. The American Mathematical Monthly, Vol. 10, No. 6/7, 1903, pp. 159-161. http://www.jstor.org/stable/ 29709359

[13] Mortini, R., Rupp, R., The Symmetric Versions of Rouché's Theorem via $\bar{\partial}$-Calculus. Journal of Complex Analysis, volume 2014 (2014). Article ID 260953, 9 pages. http://dx.doi.org/10. $1155 / 2014 / 26095366$

[14] Narasimhan, R., Nievergel, Y., Complex Analysis in One Variable. Birkhauser, Boston, 2nd Edition, 2001. 25, 70

[15] Oliveira, O. R. B de., A Complex Primitive Nth Root Of Unity: A Very Elementary Approach. arXiv: 1402.4038v1 [math.CV], 2014. http://arxiv.org/pdf/1402.4038.pdf 9

[16] Oliveira, O. R. B de., The Fundamental Theorem of Algebra: An Elementary and Direct Proof. Springer Science - Business Media, LLC, Volume 33, Number 2, 2011. x, 4 
[17] Oliveira, O. R. B de., The Fundamental Theorem of Algebra: From The Four Basic Operations. Mathematical Association of America, 2012. 4, 7

[18] Oliveira, O. R. B de., Some Simplifications in Basic Complex Analysis. arXiv: 1207.3553v2 [math.CV], 2012. 12, 25, 72

[19] Oliveira, O. R. B de., Some Simplications in the Presentations of Complex Power Series and Unordered Sums. arXiv: 1207.1472v2 [math.CV], 2012. 18, 21, 25, 40

[20] Ovchinnikov, S., Number Systems: An Introduction to Algebra and Analysis. American Mathematical Society, 2015. 4

[21] Ponnusamy, S., Silverman, H., Complex Variables with Applications. Birkhauser, Boston, 2006. 80

[22] Porcelli, P., Weiner, L. M., A derivation of Cauchy's Inequality for Polynomials. Revista de Matematica y Fisica Teorica, Vol. 11, 1957, pp. 25-28.

[23] Remmert, R., Classical Topics in Complex Function Theory. Springer - Verlag, New York, 1997. 72,90

[24] Remmert, R., Theory of Complex Functions. Springer - Verlag, New York, 1991. 12

[25] Theobald, T., Iliman, S., Einführung in die computerorientierte Mathematik mit Sage. Springer Studium Mathematik - Bachelor, 2016. 4

[26] Walsh, J. L., A Mean value theorem for polynomials and harmonic polynomials. Bulletin American Mathematical Society. 42 (1936) 923-936. http://dx.doi.org/10.1090/ S0002-9904-1936-06468-2 12

[27] Walsh, J. L., History of the Riemann Mapping Theorem. The American Mathematical Monthly, Vol. 80, No. 3 (Mar., 1973), pp. 270-276 Published by: Mathematical Association of America http://www.jstor.org/stable/2318448 72

[28] Whyburn, G. T., The Cauchy inequality in topological analysis. Proc. Natl. Acad. Sci. USA 48, (1962), 1335-1336. http://dx.doi.org/10.1073/pnas.48.8.1335 25, 43 